PHYSICAL REVIEW D 93, 044052 (2016)

\title{
Unified limiting form of graviton radiation at extreme energies
}

\author{
Marcello Ciafaloni* \\ Dipartimento di Fisica, Università di Firenze Via Sansone 1, 50019 Sesto Fiorentino, Italy \\ Dimitri Colferai ${ }^{\dagger}$ and Francesco Coradeschi ${ }^{\ddagger}$ \\ Dipartimento di Fisica, Università di Firenze and INFN, Sezione di Firenze Via Sansone 1, \\ 50019 Sesto Fiorentino, Italy \\ Gabriele Veneziano ${ }^{\S}$ \\ Collège de France, 11 place M. Berthelot, 75005 Paris, France; \\ Theory Division, CERN, CH-1211 Geneva 23, Switzerland; \\ and Dipartimento di Fisica, Università di Roma La Sapienza, Rome 00185, Italy \\ (Received 18 December 2015; published 18 February 2016)
}

\begin{abstract}
We derive the limiting form of graviton radiation in gravitational scattering at trans-Planckian energies $\left(E \gg M_{P}\right)$ and small deflection angles. We show that-owing to the graviton's spin 2-such a limiting form unifies the soft and Regge regimes of emission, by covering a broad angular range, from forward fragmentation to the deeply central region. The single-exchange emission amplitudes have a nice expression in terms of the transformation phases of helicity amplitudes under rotations. As a result, the multiple-exchange emission amplitudes can be resummed via an impact parameter $b$-space factorization theorem that takes into account all coherence effects. We then see the emergence of an energy spectrum of the emitted radiation which, being tuned on $\hbar / R \sim M_{P}^{2} / E \ll M_{P}$, is reminiscent of Hawking's radiation. Such a spectrum is much softer than the one naïvely expected for increasing input energies and neatly solves a potential energy crisis. Furthermore, by including rescattering corrections in the (quantum) factorization formula, we are able to recover the classical limit and find the corresponding quantum corrections. Perspectives for the extrapolation of such limiting radiation towards the classical collapse regime (where $b$ is of the order of the gravitational radius $R$ ) are also discussed.
\end{abstract}

DOI: 10.1103/PhysRevD.93.044052

\section{INTRODUCTION}

The thought experiment of trans-Planckian-energy gravitational scattering has been investigated, since the eighties [1-7], as a probe of quantum-gravity theories, mostly in connection with the problem of a possible loss of quantum coherence in a process leading classically to gravitational collapse. In an $S$-matrix framework such a loss would be associated with the breakdown of unitarity at sufficiently small impact parameters.

In the scattering regime of large energies $\left(\sqrt{s} \gg M_{P}\right)$ but small deflection angles (i.e., in a regime far away from that of collapse), several authors proposed [1-5], on various grounds, an approximate semiclassical description, whose $S$-matrix exponentiates, at fixed impact parameter, an eikonal function of order $\alpha_{G} \equiv G s / \hbar \gg 1$, which is simply

\footnotetext{
*ciafaloni@fi.infn.it

†colferai@fi.infn.it

coradeschi@fi.infn.it

§abriele.veneziano@cern.ch
}

Published by the American Physical Society under the terms of the Creative Commons Attribution 3.0 License. Further distribution of this work must maintain attribution to the author(s) and the published article's title, journal citation, and DOI. related to graviton exchanges at large impact parameters $b \gg R \equiv 2 G \sqrt{s}$. Such a description has its classical counterpart in the scattering of two Aichelburg-Sexl (AS) shock waves [8].

Starting from that leading eikonal approximation, the strategy followed in $[6,7]$ consisted in a systematic study of subleading corrections to the eikonal phase, scattering angle, and time delays [9-11] in terms of the expansion parameter $R^{2} / b^{2}$ (and $l_{s}^{2} / b^{2}$ if working within string theory). These corrections can be resummed, in principle, by solving a classical field theory and one can thus study the critical region $b \sim R$ where gravitational collapse is expected.

This program was carried out, neglecting string corrections and after a drastic truncation of the classical field theory due to Lipatov [12], in [13] (see also [14-16]). It was noted there that below some critical impact parameter value $b_{c} \sim R$ (in good agreement with the expected classical critical value [17-20]), the $S$ matrix-evaluated by taking UV-safe (regular), but possibly complex, solutions of the field equations-shows a unitarity deficit. This was confirmed, at the quantum level, by a tunneling interpretation of such restricted solutions [21-23]. The above results suggest that the lost information could possibly be recovered only through use of UV-sensitive solutions which, by 
definition, cannot be studied by the effective-action approach of [13] and remain to be investigated on the basis of the underlying (string) theory itself. It is also possible, of course, that the apparent loss of unitarity is caused instead by the drastic truncation made in [13] of Lipatov's effective field theory [12].

On the other hand, the parallel investigation of gravitational radiation associated with trans-Planckian scattering brought a worrisome surprise: even if such radiation is pretty soft, $\langle q\rangle \simeq \hbar / b$ being its typical transverse momentum, its rapidity density $\sim \alpha_{G}$ is so large as to possibly endanger energy conservation [24,25], at least in the early naïve extrapolations of the available rapidity phase space $[13,16]$. Energy conservation can be enforced by hand, the result being that the flat low-energy spectrum (predicted by known zero-frequency-limit theorems [26]) extends up to a cutoff at $\omega \sim b^{2} / R^{3}$. But that would mean that a fraction $O(1)$ of the initial energy is emitted in gravitational radiation already at scattering angles $O\left(\alpha_{G}^{-1 / 2}\right) \ll 1$, something rather hard to accept.

This unexpected result prompted the study of the purely classical problem of gravitational bremsstrahlung in ultrarelativistic, small-angle gravitational scattering, a subject pioneered in the seventies by Peter D'Eath and collaborators $[27,28]$ and by Kovacs and Thorne [29,30]. Those papers, however, were rather inconclusive about the ultrarelativistic limit (the method of Refs. [29,30], for instance, does not apply to scattering angles larger than $m / E \equiv \gamma^{-1}$, and thus, in particular, to our problem). Nonetheless, two groups of authors [31,32] managed to discuss directly the massless limit of the classical bremsstrahlung problem showing the absence of an energy crisis and the emergence of a characteristic frequency scale of order $R^{-1}$ beyond which the emitted-energy spectrum is no longer flat (within the approximations used in [31] the spectrum decreases like $\omega^{-1}$ till the approximation breaks down at $\left.\omega \sim b^{2} / R^{3}\right)$. These classical results called for a more careful investigation of the quantum problem.

And indeed the good surprise was that-after a careful account of matrix elements, phases, and coherence effects-the limiting form of such radiation for $\alpha_{G} \gg 1$ takes a simple and elegant expression and has the unique feature of unifying two well-known limits of emission amplitudes: the soft and the Regge limit. As a consequence, besides reducing in a substantial way the total emittedenergy fraction, the spectrum drifts towards characteristic energies of order $\hbar / R \sim M_{P}^{2} / E \ll M_{P}$, much smaller than those expected from the naïve Regge behavior, and reminiscent of Hawking's radiation [33] (see also [34]) from a black hole of mass $E$. That nice surprise, which we illustrate here in full detail, has been presented recently in a short paper [35].

We should note incidentally that, in a different but related investigation of trans-Planckian graviton production integrated over impact parameter, a similarly surprising feature was found (even more surprisingly by a tree-level calculation) in [36], the typical energy of the emitted gravitons being again of order $\hbar / R$, with a very large multiplicity of order $s / M_{P}^{2}$ i.e. of a black hole entropy for $M \sim \sqrt{s}$.

The above list of surprises points in the direction of a more structural role of the gravitational radius in the radiation problem, rather than in the scattering amplitude calculation itself, so that approaching the collapse region at quantum level may actually be easier and more informative if made from the point of view of the radiation associated with the scattering process.

One may wonder what the deep reason is for all that. Here we show that our unified limiting form of radiation, at the first subleading level in the parameter $R^{2} / b^{2}$, is due to the dual role of the graviton spin two: on the one hand it determines, by multigraviton exchanges, the leading AS metric associated with the colliding particles as well as its radiative components at first subleading level; on the other hand, it also determines the transformation properties of the emission amplitudes for definite helicity final states. These, in turn, are closely connected to the emission currents themselves.

For the above reasons-after a brief introduction to eikonal scattering in Sec. II-we emphasize (Sec. III) the physical matrix elements of the relevant emission currents whose phases - due to the absence of collinear singularities in gravity-play a crucial role in both the soft and the Regge regimes. The unified form of graviton emission is then determined - at the single-exchange level—by matching the soft and Regge behaviors in all relevant angular regions, from nearly forward fragmentation to deeply central emission. The resulting expressions are just the Fourier transforms of two different components of the radiative metric tensor, which, however, yield identical results because of a transversality condition.

The next step in the construction of the emission amplitudes is to resum the contributions of all the graviton exchanges that occur during eikonal scattering. This is done in Sec. IV, by establishing a $b$-factorization theorem for each single-exchange contribution, and by summing them up with the appropriate phases due to the dependence of the helicity amplitudes on the incidence direction. The outcome, already presented in [35], has a classical limit that resembles (but slightly differs from) the one of [31]. An important new result of this work is that, by also taking into account rescattering of the emitted gravitons all over the eikonal evolution, the classical limit of [31] is fully recovered together with some (or perhaps all) quantum corrections to it. This resummation yields a coherent average over incidence directions, up to the Einstein deflection angle $\Theta_{s}(b)=2 R / b$, providing important (de) coherence effects which tend to suppress frequencies of order $\omega>R^{-1}$. The above procedure is finally generalized to multiple emissions by constructing the appropriate (unitary) coherent-state operator. 
The spectrum is then described and analyzed in Sec. V, both in frequency and in angular distribution. This is done, in this paper, by taking into account the incidence angle dependence only. Including rescattering effects, both at the classical and quantum level, is deferred to a later work. The ensuing perspectives for the development of the present method towards the classical collapse region (given in Sec. VI) are based on the new features of the resummation pointed out in this paper, which are typical of the emitted gravitational radiation associated with trans-Planckian scattering. Finally, a number of detailed calculations and useful remarks are left to the appendixes.

\section{TRANS-PLANCKIAN EIKONAL SCATTERING}

Throughout this paper, as in [13], we restrict our attention to collisions in four-dimensional space-time and in the point-particle (or quantum field theory) limit. Consider first the elastic gravitational scattering $p_{1}+p_{2} \rightarrow$ $p_{1}^{\prime}+p_{2}^{\prime}$ of two ultrarelativistic particles, with external momenta parametrized as

$$
p_{i}=E_{i}\left(1, \boldsymbol{\Theta}_{i}, \sqrt{1-\left|\boldsymbol{\Theta}_{i}\right|^{2}}\right),
$$

at center-of-mass energy $2 E=\sqrt{s} \gg M_{P}$ and momentum transfer $Q^{\mu} \equiv p_{1}^{\prime \mu}-p_{1}^{\mu}=p_{2}^{\mu}-p_{2}^{\prime \mu}$ with transverse component $\boldsymbol{Q}=E \boldsymbol{\Theta}_{s}$; the 2-vectors $\boldsymbol{\Theta}_{i}=\left|\boldsymbol{\Theta}_{i}\right|\left(\cos \phi_{i}, \sin \phi_{i}\right)$ describe both azimuth $\phi_{i}$ and polar angles $\left|\boldsymbol{\Theta}_{i}\right| \ll 1$ of the corresponding 3-momentum with respect to the longitudinal $z$ axis.

This regime is characterized by a strong effective coupling $\alpha_{G} \equiv G s / \hbar \gg 1$ and was argued by several authors $[1,2,4,6]$ to be described by an all-order leading approximation which has a semiclassical effective metric interpretation. The leading result for the $S$-matrix $S(b, E)$ in impact-parameter $b \equiv J / E$ space has the eikonal form

$$
\begin{aligned}
S(b, E) & =\exp \left[2 \mathrm{i} \delta_{0}(b, E)\right], \\
\delta_{0}(b, E) & =\alpha_{G} \log \frac{L}{b},
\end{aligned}
$$

$L$ being a factorized - and thus irrelevant-IR cutoff.

Corrections to the leading form (2.2) involve additional powers of the Newton constant $G$ in two dimensionless combinations

$$
\frac{\hbar G}{b^{2}}=\frac{l_{P}^{2}}{b^{2}}, \quad \frac{4 G^{2} s}{b^{2}}=\frac{R^{2}}{b^{2}} \sim \alpha_{G} \frac{l_{P}^{2}}{b^{2}} \gg \frac{l_{P}^{2}}{b^{2}},
$$

$l_{P} \equiv \sqrt{\hbar G}$ being the Planck length. Since $\alpha_{G} \gg 1$ we can neglect completely the first kind of corrections. Furthermore, we can consider the latter within a perturbative framework since the impact parameter $b$ is much larger than the gravitational radius $R \equiv 2 G \sqrt{s}$.
In order to understand the scattering features implied by (2.2) we can compute the $\boldsymbol{Q}$-space amplitude

$$
\begin{aligned}
\frac{1}{S} M_{\text {eik }}\left(s, Q^{2}\right) & =4 \int \mathrm{d}^{2} \boldsymbol{b} \mathrm{e}^{-\frac{\mathrm{i} b \cdot \boldsymbol{Q}}{\hbar}} \frac{\mathrm{e}^{2 \mathrm{i} \delta_{0}(b, E)}}{2 \mathrm{i}} \\
& =\frac{8 \pi \alpha_{G}}{\boldsymbol{Q}^{2}}\left(\frac{4 \hbar^{2}}{\boldsymbol{Q}^{2} L^{2}}\right)^{-\mathrm{i} \alpha_{G}} \frac{\Gamma\left(1-\mathrm{i} \alpha_{G}\right)}{\Gamma\left(1+\mathrm{i} \alpha_{G}\right)},
\end{aligned}
$$

where the expression in the last line is obtained strictly speaking by extending the $\boldsymbol{b}$ integration up to small $|\boldsymbol{b}| \lesssim R$ [1], where corrections may be large. But it is soon realized that the $\boldsymbol{b}$ integration in (2.4) is dominated by the saddlepoint

$$
\boldsymbol{Q}=E \boldsymbol{\Theta}_{s}(\boldsymbol{b})=-E \frac{2 R}{b} \hat{\boldsymbol{b}}=-\alpha_{G} \frac{\hbar}{b} \hat{\boldsymbol{b}},
$$

which leads to the same expression for the amplitude, apart from an irrelevant $\boldsymbol{Q}$-independent phase factor. The saddlepoint momentum transfer (2.5) comes from a large number $\langle n\rangle \sim \alpha_{G}$ of graviton exchanges (Fig. 1), corresponding to single-hit momentum transfers $\left\langle\left|\boldsymbol{q}_{j}\right|\right\rangle \simeq \hbar / b$ which are small, with very small scattering angles $\left|\boldsymbol{\theta}_{j}\right|$ of order $\theta_{m} \simeq \hbar /(b E)$. The overall scattering angle, though small for $b \gg R$, is much larger than $\theta_{m}$ and is $\left|\boldsymbol{\Theta}_{s}\right|=2 R / b=$ $2 \alpha_{G} \theta_{m}$, the Einstein deflection angle.

In other words, every single hit is effectively described by the elastic amplitude

$$
M_{\mathrm{el}}\left(\boldsymbol{Q}_{j}\right)=\frac{\kappa^{2} s^{2}}{\boldsymbol{Q}_{j}^{2}}=\frac{\kappa^{2} s^{2}}{E^{2} \boldsymbol{\theta}_{j}^{2}}, \quad\left(\kappa^{2}=\frac{8 \pi G}{\hbar}\right),
$$

which is in turn directly connected to the phase shift $\delta_{0}$ :

$$
\begin{aligned}
& \delta_{0}(E,|\boldsymbol{b}|)=\frac{1}{4 s} \int \frac{\mathrm{d}^{2} \boldsymbol{Q}}{(2 \pi)^{2}} \mathrm{e}^{\mathrm{i} \frac{\mathrm{Q} \cdot \boldsymbol{b}}{\hbar}} M_{\mathrm{el}}(\boldsymbol{Q}) \\
& =\alpha_{G} \int \frac{\mathrm{d}^{2} \boldsymbol{\theta}_{s}}{2 \pi \boldsymbol{\theta}_{s}^{2}} \mathrm{e}^{\mathrm{i} \frac{\mathrm{i} \boldsymbol{\theta}_{S} \boldsymbol{b}}{\hbar}} .
\end{aligned}
$$

The relatively soft nature of trans-Planckian scattering just mentioned is also-according to [4] - the basis for its validity in the string-gravity framework. In fact, string

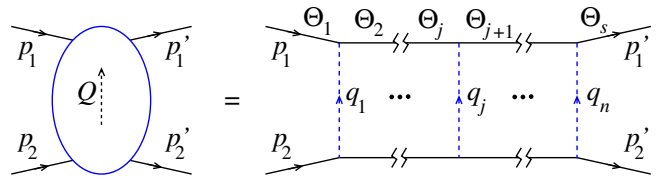

FIG. 1. The scattering amplitude of two trans-Planckian particles (solid lines) in the eikonal approximation. Dashed lines represent (Reggeized) graviton exchanges. The fast particles propagate on shell throughout the whole eikonal chain. The angles $\boldsymbol{\Theta}_{j} \simeq \sum_{i=1}^{j-1} \boldsymbol{\theta}_{i}$ denote the direction of particle 1 with respect to the $z$ axis along the scattering process. 
theory yields exponentially suppressed amplitudes in the high-energy, fixed-angle limit [37] so that several softer hits may be preferred to a single hard one in the $b \gg R$ regime. Furthermore, this procedure can be generalized to multiloop contributions in which the amplitude, for each power of $G$, is enhanced by additional powers of $s$, due to the dominance of $s$-channel iteration in high-energy spin-2 exchange versus the $t$-channel one (which provides at most additional powers of $\log s$ ). That is the mechanism by which the $S$ matrix exponentiates an eikonal function (or operator) with the effective coupling $\alpha_{G} \equiv G s / \hbar$ and subleading contributions which are a power series in $R^{2} / b^{2}$ (and/or $l_{s}^{2} / b^{2}$ ). Finally, the scattering is self sustained by the saddle point (2.5), so that string effects themselves may be small —and are calculable [4,6]—if $b \gg R, l_{s} \gg l_{P}$ and even at arbitrary $b$ if $R \ll l_{s}$.

Both the scattering angle (2.5) [and the $S$ matrix (2.2)] can be interpreted from the metric point of view [1] as the geodesic shift (and the quantum matching condition) of a fast particle in the AS metric [8] of the other.

More directly, the associated metric emerges from the calculation [9] of the longitudinal fields coupled to the incoming particles in the eikonal series, which turn out to be

$$
\begin{gathered}
\frac{1}{4} h^{++}=h_{--}=2 \pi R a_{0}(\boldsymbol{x}) \delta\left(x^{-}-\pi R \epsilon\left(x^{+}\right) a_{0}(b)\right), \\
a_{0}(\boldsymbol{x})=\frac{1}{2 \pi} \log \frac{L^{2}}{\boldsymbol{x}^{2}}, \quad \delta_{0}(b, E)=\pi \alpha_{G} a_{0}(b) .
\end{gathered}
$$

Such shock-wave expressions yield two AS metrics for the fast particles, as well as the corresponding time delay and trajectory shifts at leading level. When $b$ decreases towards $R \gg l_{s}$, corrections to the eikonal and to the effective metric involving the $R^{2} / b^{2}$ parameter have to be included, as well as graviton radiation, to which we now turn.

\section{LIMITING FORM OF EMISSION FROM SINGLE-GRAVITON EXCHANGE}

The basic emission process $p_{1}+p_{2} \rightarrow p_{1}^{\prime}+p_{2}^{\prime}+q$ at tree level (Fig. 2) of a graviton of momentum $q^{\mu}: \boldsymbol{q}=\hbar \omega \boldsymbol{\theta}$ yields simple, and yet interesting, amplitudes in various angular regimes (Fig. 3) that we now consider, assuming a relatively soft emission energy $\hbar \omega \ll E$. Note that this restriction still allows for a huge graviton phase space,

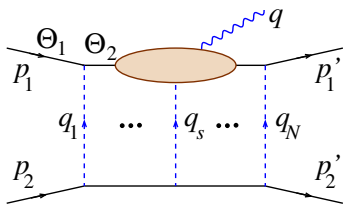

(a)

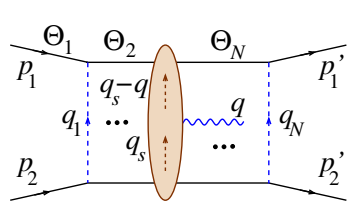

(b)
FIG. 2. Picture and notation of generic exchange emission in (a) the soft and (b) the Regge limits.

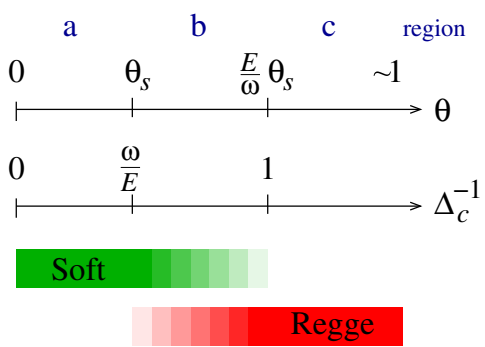

FIG. 3. Schematics of dynamical regimes of graviton emission in trans-Planckian scattering with single-exchange (here $\hbar=1$ ). $\Delta_{c}$ is a parameter used in Sec. III C.

corresponding to classical frequencies potentially much larger than the characteristic scale $R^{-1}$, due to the large gravitational charge $\alpha_{G} \equiv G s / \hbar \gg 1$. We consider three regimes:

(a) The regime $\left|\boldsymbol{\theta}_{s}\right|>|\boldsymbol{\theta}|$ (where $\left|\boldsymbol{\theta}_{s}\right|=\left|\boldsymbol{q}_{s}\right| / E$ is the single-hit scattering angle) is characterized by relatively small emission angles and subenergies. If scattering is due to a single exchange at impact parameter $b$, then $\left|\boldsymbol{\theta}_{s}\right| \sim \hbar / E b \equiv \theta_{m}$ and $\vec{q}$ is nearly collinear to $\vec{p}_{1}$. In that region the amplitude is well described by external-line insertions, but turns out to be suppressed because of helicity conservation zeroes.

(b) $|\boldsymbol{\theta}|>\left|\boldsymbol{\theta}_{s}\right|>\frac{\hbar \omega}{E}|\boldsymbol{\theta}|$. In this regime the subenergies reach the threshold of high-energy (Regge) behavior, still remaining in the validity region of external-line insertions, due to the condition $\left|\boldsymbol{q}_{s}\right|=E\left|\boldsymbol{\theta}_{s}\right|>$ $\hbar \omega|\boldsymbol{\theta}|=|\boldsymbol{q}|$ which suppresses insertions on exchanged graviton lines.

(c) Finally, in the regime $\left|\boldsymbol{\theta}_{s}\right|<\frac{\hbar \omega}{E}|\boldsymbol{\theta}|$ the soft approximation breaks down in favor of the (high-energy) H-diagram amplitude [6] which contains internal-line insertions also [12].

\section{A. Soft amplitudes in the Weinberg limit}

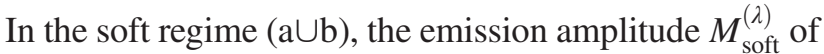
a graviton with momentum $q$ and helicity (or polarization) $\lambda$ can be expressed as the product of the elastic amplitude $M_{\mathrm{el}}=\kappa^{2} s^{2} / Q^{2}$ and the external-line insertion factor $J_{W}^{(\lambda)} \equiv$ $J_{W}^{\mu \nu} \epsilon_{\mu \nu}^{(\lambda) *}$, where $\epsilon_{\mu \nu}^{(\lambda)}$ is the polarization tensor of the emitted graviton and $J_{W}^{\mu \nu}$ is the Weinberg current [38] $\left[\eta_{i}=+1(-1)\right.$ for incoming (outgoing) lines]

$$
\begin{aligned}
J_{W}^{\mu \nu} & =\kappa \sum_{i} \eta_{i} \frac{p_{i}^{\mu} p_{i}^{\nu}}{p_{1} \cdot q} \\
& =\kappa\left(\frac{p_{1}^{\mu} p_{1}^{\nu}}{p_{1} \cdot q}-\frac{p_{1}^{\prime \mu} p_{1}^{\prime \nu}}{p_{1}^{\prime} \cdot q}+\frac{p_{2}^{\mu} p_{2}^{\nu}}{p_{2} \cdot q}-\frac{p_{2}^{\prime \mu} p_{2}^{\prime \nu}}{p_{2}^{\prime} \cdot q}\right)
\end{aligned}
$$

and was already discussed in the Planckian framework in [6]. 
We are interested in the projections of the Weinberg current over states of definite positive/negative helicity, which can be conveniently defined by

$$
\begin{aligned}
& \epsilon_{ \pm}^{\mu \nu}=\frac{1}{\sqrt{2}}\left(\epsilon_{T T}^{\mu \nu} \pm \mathrm{i} \epsilon_{L T}^{\mu \nu}\right)=\frac{1}{2}\left(\epsilon_{T}^{\mu} \pm \mathrm{i} \epsilon_{L}^{\mu}\right)\left(\epsilon_{T}^{\nu} \pm \mathrm{i} \epsilon_{L}^{\nu}\right), \\
& \epsilon_{T T}^{\mu \nu}=\frac{1}{\sqrt{2}}\left(\epsilon_{T}^{\mu} \epsilon_{T}^{\nu}-\epsilon_{L}^{\mu} \epsilon_{L}^{\nu}\right), \quad \epsilon_{L T}^{\mu \nu}=\frac{1}{\sqrt{2}}\left(\epsilon_{L}^{\mu} \epsilon_{T}^{\nu}+\epsilon_{T}^{\mu} \epsilon_{L}^{\nu}\right) \\
& \epsilon_{T}^{\mu}=\left(0,-\varepsilon_{i j} \frac{q_{j}}{|\boldsymbol{q}|}, 0\right), \quad \epsilon_{L}^{\mu}=\left(\frac{q^{3}}{|\boldsymbol{q}|}, \mathbf{0}, \frac{q^{0}}{|\boldsymbol{q}|}\right) \mp \frac{q^{\mu}}{|\boldsymbol{q}|}
\end{aligned}
$$

with $\varepsilon_{12}=1$ and the - and + signs in $\epsilon_{L}^{\mu}$ corresponding to a graviton emission in the forward and backward hemisphere respectively.

By referring, for definiteness, to the forward hemisphere, we define the momentum transfers $\boldsymbol{q}_{1(2)} \equiv \boldsymbol{p}_{1(2)}-\boldsymbol{p}_{1(2)}^{\prime}$, $\boldsymbol{q}=\boldsymbol{q}_{1}+\boldsymbol{q}_{2}$, and the scattering angle $\boldsymbol{q}_{2} \equiv \boldsymbol{E} \boldsymbol{\theta}_{s}$, and restrict ourselves to the forward region $|\boldsymbol{\theta}|,\left|\boldsymbol{\theta}_{s}\right| \ll 1$. Giving for ease of notation the results for a single helicity, a delicate but straightforward calculation (Appendix A) leads to the following explicit result in the c.m. frame with $\boldsymbol{p}_{1}=0$ :

$J_{W-}\left(q^{3}>0 ; \boldsymbol{\theta}, \boldsymbol{\theta}_{s}\right)=\frac{J_{W}}{\sqrt{2}}=\kappa \frac{E}{\hbar \omega}\left(\mathrm{e}^{2 \mathrm{i}\left(\phi_{\theta-\theta_{s}}-\phi_{\theta}\right)}-1\right)$,

leading to a factorized soft emission amplitude

$$
\begin{aligned}
M_{\text {soft }}\left(\boldsymbol{\theta}_{s} ; E, \omega, \boldsymbol{\theta}\right) & =M_{\mathrm{el}}(E, \boldsymbol{Q}) J_{W}\left(\frac{E}{\hbar \omega}, \boldsymbol{\theta}, \boldsymbol{\theta}_{s}\right) \\
& =\kappa^{3} s^{2} \frac{1}{E \hbar \omega \boldsymbol{\theta}_{s}^{2}}\left(\mathrm{e}^{2 \mathrm{i}\left(\phi_{\theta-\theta_{s}}-\phi_{\theta}\right)}-1\right),
\end{aligned}
$$

where $|\boldsymbol{Q}|$ can be unambiguously identified with $\left|\boldsymbol{q}_{2}\right|$ in the (a) and (b) regions where Eq. (3.4) is justified.

The simple expression (3.4) shows a $1 / \omega$ dependence, but no singularities at either $\boldsymbol{\theta}=0$ or $\boldsymbol{\theta}=\boldsymbol{\theta}_{s}$ as we might have expected from the $p_{i} \cdot q$ denominators occurring in (3.1). This is due to the helicity conservation zeros in the physical projections of the tensor numerators in (3.1). Therefore, there is no collinear enhancement of the amplitude in region (a) with respect to region (b), while we expect sizeable corrections to it in region (c), where internal insertions are important. The helicity phase transfer in Eq. (3.5) has a suggestive interpretation, made manifest by introducing a " $z$ representation" (proven in Appendix A 3)

$$
\begin{aligned}
\mathrm{e}^{2 \mathrm{i} \phi_{\theta}}-\mathrm{e}^{2 \mathrm{i} \phi_{\theta^{\prime}}}= & -2 \int \frac{\mathrm{d}^{2} z}{2 \pi z^{* 2}}\left(\mathrm{e}^{\mathrm{i} A z \cdot \theta}-\mathrm{e}^{\mathrm{i} A z \cdot \theta^{\prime}}\right), \\
& \left\{\begin{array}{l}
z=x+\mathrm{i} y \\
z=(x, y)
\end{array}\right.
\end{aligned}
$$

as an integral between initial and final directions in the transverse $z$ plane of the complex component of the Riemann tensor [31] in the AS metric of the incident particles.

For our analysis we need to work both in momentum and in impact parameter space. We define $\boldsymbol{b}$-space amplitudes, following the normalization convention ${ }^{1}$ of [13] and [35], as

$$
\mathcal{M}(\boldsymbol{b}) \equiv \frac{1}{(2 \pi)^{3 / 2}} \int \frac{\mathrm{d}^{2} \boldsymbol{q}_{2}}{(2 \pi)^{2}} \mathrm{e}^{\mathrm{i} \boldsymbol{q}_{2} \cdot \boldsymbol{b}} \frac{1}{4 s} M\left(\boldsymbol{q}_{2}\right),
$$

so that, in the soft case, we have

$$
\begin{aligned}
& \mathcal{M}_{\text {soft }}(\boldsymbol{b} ; E, \omega, \boldsymbol{\theta}) \\
& \quad=\sqrt{\alpha_{G}} \frac{R}{\pi} \frac{E}{\hbar \omega} \int \frac{\mathrm{d}^{2} \boldsymbol{\theta}_{s}}{2 \pi \boldsymbol{\theta}_{s}^{2}} \mathrm{e}^{\mathrm{i} \frac{\mathrm{E}}{\hbar} \boldsymbol{\theta}_{s} \cdot \boldsymbol{b}} \frac{1}{2}\left(\mathrm{e}^{2 \mathrm{i}\left(\phi_{\theta-\theta_{s}}-\phi_{\theta}\right)}-1\right) .
\end{aligned}
$$

This definition is generalized to the backward hemisphere (jet 2) by setting $E \boldsymbol{\theta}_{s}=-\boldsymbol{q}_{1}$ and $\boldsymbol{q}_{2}=E \boldsymbol{\theta}_{s}+\boldsymbol{q}$, and by using the corresponding current projections (Appendix A)

$$
\begin{aligned}
J_{W-}\left(-q^{3} ;-\boldsymbol{\theta}, \boldsymbol{\theta}_{s}\right) & =J_{W-}\left(q^{3} ; \boldsymbol{\theta}, \boldsymbol{\theta}_{s}\right)^{*} \\
& =J_{W+}\left(q^{3} ; \boldsymbol{\theta}, \boldsymbol{\theta}_{s}\right)
\end{aligned}
$$

to obtain the helicity symmetry relation between backward and forward jets

$$
\mathcal{M}_{-}\left(\boldsymbol{b} ;-q^{3},-\boldsymbol{q}\right)=\mathcal{M}_{+}\left(\boldsymbol{b} ; q^{3}, \boldsymbol{q}\right) \mathrm{e}^{-\mathrm{i} \boldsymbol{b} \cdot \boldsymbol{q}},
$$

where

$$
\begin{aligned}
\mathcal{M}_{+} & =\sqrt{\alpha_{G}} \frac{R}{\pi} \frac{E}{\hbar \omega} \int \frac{\mathrm{d}^{2} \boldsymbol{\theta}_{s}}{2 \pi \boldsymbol{\theta}_{s}^{2}} \mathrm{e}^{\mathrm{i} \frac{E}{\hbar} \boldsymbol{\theta}_{s} \cdot \boldsymbol{b}} \frac{1}{2}\left(\mathrm{e}^{-2 \mathrm{i}\left(\phi_{\theta-\theta_{s}}-\phi_{\theta}\right)}-1\right) \\
& =\mathcal{M}_{-}\left(\boldsymbol{b} ; q^{3},-\boldsymbol{q}\right)^{*} .
\end{aligned}
$$

Note the translational parameter $\mathrm{e}^{-\mathrm{i} b \cdot \boldsymbol{q}}$ (recalling that particle 2 is located at $\boldsymbol{x}=\boldsymbol{b}$ ).

Let us examine the behavior of Eqs. (3.5) and (3.8) in the various regimes (we set $\hbar=1$ for simplicity in most of Sec. III, except when needed for physical understanding). It is useful to write Eq. (3.5) in complex notation $\left(\theta \equiv|\boldsymbol{\theta}| \mathrm{e}^{\mathrm{i} \phi_{\theta}}\right.$, etc.) as

$$
M_{\mathrm{soft}}=\frac{\kappa^{3} s^{2}}{E \omega}\left(\frac{\theta}{\theta_{s}}-\frac{\theta^{*}}{\theta_{s}^{*}}\right) \frac{1}{\theta\left(\theta-\theta_{s}\right)^{*}},
$$

which implies the approximate behavior in the (a) and (b) regions

\footnotetext{
${ }^{1}$ The customary helicity amplitudes with phase space $\mathrm{d}^{3} \vec{q} /\left(\hbar^{3} 2 \omega\right)$ are given by $\mathcal{M}(\boldsymbol{b}, \boldsymbol{q})\left(\mathcal{M}(\boldsymbol{b},-\boldsymbol{q})^{*}\right)$ for helicity $-(+)$ respectively.
} 


\begin{tabular}{|c|c|c|}
\hline $\mathbf{0}$ & $\Theta_{i}=\theta_{1}$ & $\Theta_{f}=\theta_{1}+\theta$ \\
\hline \multirow[t]{2}{*}{$p_{1}$} & $k_{1}$ & $p_{1}^{\prime}$ \\
\hline & $q_{1}$ & $q_{2}$ \\
\hline$p_{2}$ & $k_{2}$ & $p_{2}^{\prime}$ \\
\hline
\end{tabular}

FIG. 4. Double-exchange process. The angles describing the direction of the right-moving fast particle during the scattering process are shown on top of the upper line.

$$
\frac{M_{\text {soft }}}{\kappa^{3} s^{2}} \simeq \begin{cases}\frac{2}{E \omega} \frac{1}{2}\left(\mathrm{e}^{2 \mathrm{i}\left(\phi_{\theta_{s}}-\phi_{\theta}\right)}-1\right) \frac{1}{\left|\theta_{s}\right|^{2}} & \left(\theta \ll \theta_{s} \Leftrightarrow \text { region } \mathrm{a}\right) \\ \frac{2}{E \omega} \mathrm{i} \sin \left(\phi_{\theta}-\phi_{\theta_{s}}\right) \frac{1}{\left|\theta \| \theta_{s}\right|} & \left(\theta \gg \theta_{s} \Leftrightarrow \text { region } \mathrm{b}\right) .\end{cases}
$$

The first behavior is typical of the IR amplitude, showing no singularities in the collinear $(\theta \rightarrow 0)$ limit, and will be relevant for our final result also.

The second behavior in (3.13), after a simple integration in $\theta_{s}$, to which only the $\cos \phi_{\theta_{s}} \sin \phi_{\theta}$ term contributes, yields the result

$$
\begin{aligned}
& \mathcal{M}_{\text {soft }}(\boldsymbol{b} ; E, \omega, \boldsymbol{\theta}) \\
& \Rightarrow \sqrt{\alpha_{G}} \frac{R}{\pi} \frac{\sin \phi_{\boldsymbol{\theta}}}{\omega b|\theta|} J_{0}(b \omega|\theta|)\left(\frac{E}{\omega} \theta_{m} \gg|\theta| \gg \theta_{m}\right)
\end{aligned}
$$

(where, for simplicity, we choose the $x$ axis in the transverse plane to be aligned with $\boldsymbol{b}$, that is $\phi_{\theta} \equiv \phi_{\theta}-\phi_{\boldsymbol{b}}$ ), which provides the most important term in the (b) region. We note that its maximum at $\phi_{\theta}=\pi / 2$ is a reminder of the collinear zeroes.

As for region (c), we already noticed that the soft evaluation breaks down there in favor of the high-energy amplitude, and in Sec. III C we substantially improve our $\boldsymbol{b}$-space amplitude in all regions by matching the soft and high-energy evaluations explicitly. The corresponding estimate, though yielding subleading corrections in region (b), will considerably change the $b|\boldsymbol{q}|>1$ behavior of Eq. (3.14).

\section{B. Amplitude transformation}

In order to compute the emission in the general case we need to establish an important point regarding the representation of the soft and Regge (see Sec. IIIC) singleexchange amplitudes. As already noted, the expression (3.5) is valid as it stands only if the initial direction (of the momentum $\vec{p}_{1}$ ) of the emitting particle is along the $z$ axis, or forms with it a small angle $\left|\boldsymbol{\Theta}_{i}\right| \ll \theta_{m}$, the single-hit large angle threshold. But as shown in Sec. II, in the eikonal evolution the fast particles scatter on average by the angle $\left|\boldsymbol{\theta}_{s}(\boldsymbol{b})\right|=2 R / b=(G s) \theta_{m} \gg \theta_{m}$, and thus we need to compute the amplitudes in the case where the emission takes place with a generic incidence angle $\boldsymbol{\Theta}_{i}$, possibly much larger than $\theta_{m}$.

Because of Lorentz invariance, we expect the $\boldsymbol{\Theta}_{i}$ dependence to occur through rotation scalars (which, in the small-angle kinematics, involve the differences $\boldsymbol{\Theta}_{i}-\boldsymbol{\Theta}_{j}$, the latter being angular 2-vectors of the fast particles), and a specific transformation phase also. The latter is in turn dependent on the definition of the helicity states $|\lambda, q, \cdots\rangle$ which is not uniquely determined. Since $\lambda$ is a Lorentz invariant for the massless graviton, such a transformation phase is only allowed by the ambiguity in relating the $q$ states to the $z$-axis state, due to the residual rotational invariance around the latter. For instance, Jacob and Wick (JW) [39] relate $q$ to $z$ by a standard rotation around the axis perpendicular to the $\langle q, z\rangle$ plane. Since such a definition is fully "body fixed" (that is, independent of external observables) we expect the JW amplitudes to be invariant for small rotations of the $z$ axis around an axis perpendicular to it, because in such a limit the rotations involved will commute. ${ }^{2}$ On the other hand, our helicity states are defined in terms of the physical polarizations in Eqs. (3.2)-(3.3), which are dependent not only on $q^{\mu}$, but also on the $z$ axis, which occurs as an external variable in the $T$ projection. We show in Appendix B that the ensuing relation to JW amplitudes is a simple multiplication by $\exp \left(\mathrm{i} \lambda \phi_{\boldsymbol{\theta}}\right)$, where the azimuthal variable is generally $\mathcal{O}(1)$. Since the $\boldsymbol{\Theta}_{i}$ rotation acts on $\boldsymbol{\theta}$ as the translation $\boldsymbol{\theta}-\boldsymbol{\Theta}_{i}$ for small polar angles, we expect the transformation phase to be nontrivial and given by $\exp \left[\mathrm{i} \lambda\left(\phi_{\boldsymbol{\theta}}-\phi_{\boldsymbol{\theta}-\boldsymbol{\Theta}_{i}}\right)\right]$, as fully proved in Appendix B and explicitly checked in Appendix A.

Therefore, in the forward region $\boldsymbol{\Theta}_{i}, \boldsymbol{\Theta}_{f}, \boldsymbol{\theta} \ll 1, \boldsymbol{\Theta}_{f}$ being the outgoing direction of the (intermediate) fast particle, the momentum-space helicity amplitudes transform as (Appendix B)

$M^{\left(\boldsymbol{\Theta}_{i}\right)}\left(\boldsymbol{\Theta}_{f}, \boldsymbol{\theta}\right)=\mathrm{e}^{\mathrm{i} \lambda\left(\phi_{\theta}-\phi_{\theta-\boldsymbol{\Theta}_{i}}\right)} M^{(\mathbf{0})}\left(\boldsymbol{\Theta}_{f}-\boldsymbol{\Theta}_{i}, \boldsymbol{\theta}-\boldsymbol{\Theta}_{i}\right)$,

where $\lambda$ is the helicity of the emitted graviton, $\lambda=-2$ in our case. In $\boldsymbol{b}$ space, i.e., by Fourier transforming with respect to $\boldsymbol{Q}=E\left(\boldsymbol{\Theta}_{f}-\boldsymbol{\Theta}_{i}\right)$, one finds

$$
\mathcal{M}^{\left(\boldsymbol{\Theta}_{i}\right)}(\boldsymbol{b}, \boldsymbol{\theta})=\mathrm{e}^{\mathrm{i} \lambda\left(\phi_{\theta}-\phi_{\theta-\boldsymbol{\Theta}_{i}}\right)} \mathcal{M}^{(\mathbf{0})}\left(\boldsymbol{b}, \boldsymbol{\theta}-\boldsymbol{\Theta}_{i}\right) .
$$

The expression (3.15) is easily argued for as a consistency requirement for the insertion of the Weinberg current on the double-exchange process (Fig. 4) in the soft limit. In fact, by the identity of Weinberg contributions $J_{p}-J_{p^{\prime}}=$ $\left(J_{p}-J_{k}\right)+\left(J_{k}-J_{p^{\prime}}\right)$ we must have

$$
J_{W}^{( \pm)}\left(\boldsymbol{\Theta}_{i}, \boldsymbol{\Theta}_{f}\right)=J_{W}^{( \pm)}\left(\mathbf{0}, \boldsymbol{\Theta}_{f}\right)-J_{W}^{( \pm)}\left(\mathbf{0}, \boldsymbol{\Theta}_{i}\right),
$$

\footnotetext{
${ }^{2}$ Our amplitude is invariant under $\mathrm{O}(1)$ rotations around the $\mathrm{z}$ axis, which can be separately considered.
} 


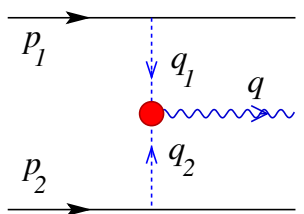

FIG. 5. Diagrammatic picture for the emission of a graviton from two fast particles scattering in the Regge limit. The blob in the middle represents the Lipatov vertex, i.e., the effective Reggeon-Reggeon-graviton coupling.

which agrees with the direct calculation of Appendix A as well, from which it follows that (note that $\boldsymbol{\theta}_{s} \simeq \boldsymbol{\Theta}_{f}-\boldsymbol{\Theta}_{i}$ for the soft amplitude)

$$
\begin{aligned}
M_{\text {soft }}^{\left(\boldsymbol{\Theta}_{i}\right)} & =\kappa^{3} s^{2} \frac{2}{E \omega \boldsymbol{\theta}_{s}^{2}} \frac{1}{2}\left(\mathrm{e}^{2 \mathrm{i}\left(\phi_{\theta-\Theta_{f}}-\phi_{\theta}\right)}-\mathrm{e}^{2 \mathrm{i}\left(\phi_{\theta-\Theta_{i}}-\phi_{\theta}\right)}\right) \\
& =\kappa^{3} s^{2} \frac{2}{E \omega} \frac{\mathrm{e}^{2 \mathrm{i}\left(\phi_{\theta-\Theta_{i}}-\phi_{\theta}\right)}}{\boldsymbol{\theta}_{s}^{2}} \frac{1}{2}\left(\mathrm{e}^{2 \mathrm{i}\left(\phi_{\theta-\boldsymbol{\theta}_{f}}-\phi_{\theta-\boldsymbol{\Theta}_{i}}\right)}-1\right),
\end{aligned}
$$

exactly as predicted by Eq. (3.15) with $\lambda=-2$. Equation (3.18) keeps the suggestive interpretation of helicity charge transfer from initial to final state in the general case.

\section{Matching of soft amplitude with the Regge limit}

As soon as the rapidity interval $2 Y_{b}=2 \log (E b / \hbar)$ between $p_{1}^{\prime}$ and $p_{2}^{\prime}$, and the relative rapidity $Y_{b}-y$ between $p_{1}^{\prime}$ and $q$ become large, high-energy emission in the Regge limit becomes relevant, as predicted by the Lipatov vertex [12] (see Fig. 5) and the H diagram [6] [see Fig. 6(a)]. More precisely, for scattering due to singlegraviton exchange, the Regge limit is relevant in the region where the graviton is emitted at a relatively large angle, $1 \gg|\theta| \gg \theta_{m} \equiv \hbar /(E b)$ (as already noted, $\left|\theta_{s}\right| \sim \theta_{m}$ in the single-hit case). This large-angle region comprises regions (b) and (c) discussed at the beginning of Sec. III and is particularly relevant for region (c) in which internal-line insertions are important.

Using the same kinematic notation as in Sec. III A, the Lipatov current is given by [12] (see also [6])

$$
\begin{aligned}
J_{L}^{\mu \nu} & =\frac{\kappa}{2}\left(J^{\mu} J^{\nu}-\left|\boldsymbol{q}_{1 \perp}\right|^{2}\left|\boldsymbol{q}_{2 \perp}\right|^{2} j^{\mu} j^{\nu}\right) \quad \text { with } \\
j^{\mu} & \equiv \frac{p_{1}^{\mu}}{p_{1} \cdot q}-\frac{p_{1}^{\mu}}{p_{2} \cdot q}, \\
J^{\mu} & \equiv\left|\boldsymbol{q}_{1 \perp}\right|^{2} \frac{p_{1}^{\mu}}{p_{1} \cdot q}-\left|\boldsymbol{q}_{2 \perp}\right|^{2} \frac{p_{2}^{\mu}}{p_{2} \cdot q}+q_{1}^{\mu}-q_{2}^{\mu}-\left|\boldsymbol{q}_{\perp}\right|^{2} j^{\mu},
\end{aligned}
$$

where $\boldsymbol{q}_{\perp 1}, \boldsymbol{q}_{\perp 2}, \boldsymbol{q}_{\perp}$ denote transverse (vectorial) components to the $\vec{p}_{1}$ direction (which of course coincide with $\boldsymbol{q}_{1}$, $\boldsymbol{q}_{2}$ and $\boldsymbol{q}$ when $\left.\boldsymbol{p}_{1}=\boldsymbol{\Theta}_{i}=0\right)$ and the corresponding graviton emission amplitude (considering again a single helicity for definiteness) is

$$
M_{\text {Regge }}=\frac{\kappa^{2} s^{2}}{\left|\boldsymbol{q}_{1 \perp}\right|^{2}\left|\boldsymbol{q}_{2 \perp}\right|^{2}} J_{L}^{\mu \nu} \epsilon_{\mu \nu}^{(-)}
$$

note that $-\left|\boldsymbol{q}_{1(2) \perp}\right|^{2}$ corresponds to the virtuality $q_{1(2)}^{\mu} q_{1(2) \mu}$ in the Regge kinematics.

More quantitatively, in the c.m. frame with zero incidence angle $\left(\boldsymbol{p}_{1}=E \boldsymbol{\Theta}_{i}=0\right)$ and in the forward region $|\boldsymbol{\theta}|,\left|\boldsymbol{\theta}_{s}\right| \ll 1$, the amplitude takes the form [13] (see also Appendix A)

$$
M_{\text {Regge }}\left(\boldsymbol{\theta} ; E, \omega, \boldsymbol{\theta}_{s}\right)=\kappa^{3} s^{2} \frac{1-\mathrm{e}^{-2 \mathrm{i}\left(\phi_{q_{2}}-\phi_{q-q_{2}}\right)}}{\boldsymbol{q}^{2}}
$$

(remember that $\boldsymbol{q}=\omega \boldsymbol{\theta}$ and $\boldsymbol{q}_{2}=E \boldsymbol{\theta}_{s}$ ). The corresponding amplitude in $\boldsymbol{b}$ space, according to the definition (3.7), is given by

$$
\begin{aligned}
& \mathcal{M}_{\text {Regge }}(\boldsymbol{b} ; E, \omega, \boldsymbol{\theta}) \\
& \quad=\sqrt{\alpha_{G}} \frac{R}{\pi} \int \frac{\mathrm{d}^{2} q_{2}}{2 \pi|\boldsymbol{q}|^{2}} \mathrm{e}^{\mathrm{i} q_{2} \cdot \boldsymbol{b}} \frac{1}{2}\left(1-\mathrm{e}^{2 \mathrm{i}\left(\phi_{q_{2}-q}-\phi_{q_{2}}\right)}\right) \\
& \equiv \sqrt{G} s \frac{R}{2} \tilde{h}(\boldsymbol{b}, \boldsymbol{q}) \\
& \equiv \sqrt{G} s \frac{R}{2} \boldsymbol{b}^{2} \int \mathrm{d}^{2} z \mathrm{e}^{\mathrm{i}|\boldsymbol{b}| \omega z \cdot \boldsymbol{\theta}} h(\boldsymbol{b},|\boldsymbol{b}| \boldsymbol{z}),
\end{aligned}
$$

where $\tilde{h}(\boldsymbol{b}, \boldsymbol{q})$ admits the integral representation $\left(b \equiv b^{1}+\mathrm{i} b^{2} \in \mathbb{C}\right)$

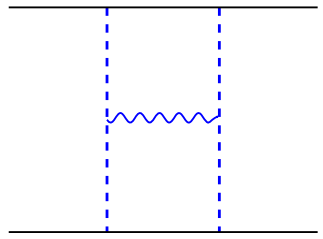

(a)

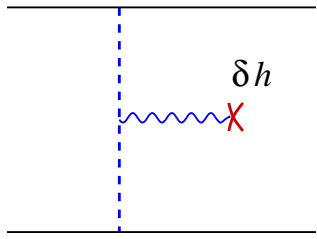

(b)

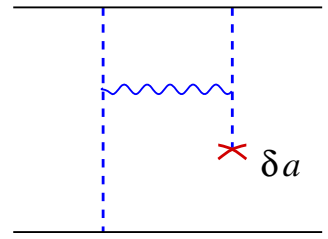

(c)

FIG. 6. (a) The H diagram, yielding the first subleading semiclassical correction to the elastic amplitude. (b)-(c) Off-shell diagrams contributing to the metric fluctuations of the $h$ and $a$ fields. 


$$
\begin{aligned}
\tilde{h}(\boldsymbol{b}, \boldsymbol{q})= & \frac{2}{\pi} \int_{0}^{\infty} \mathrm{d} \eta \eta \mathrm{e}^{-\eta} \\
& \times\left[\frac{1}{b \omega \theta^{*}\left(b \omega \theta^{*}-2 \mathrm{i} \eta\right)}+\frac{\mathrm{e}^{\mathrm{i} \omega\left(b^{*} \theta+b \theta^{*}\right) / 2}}{b^{*} \omega \theta\left(b^{*} \omega \theta+2 \mathrm{i} \eta\right)}\right],
\end{aligned}
$$

and turns out to be equal to the Fourier transform with respect to $\boldsymbol{q}$ of the $H$-diagram field. The latter's expression in the space of the transverse coordinate $\boldsymbol{x}=\left(x^{1}, x^{2}\right)$ of the emitted graviton is

$$
\begin{aligned}
h(\boldsymbol{b}, \boldsymbol{x}) & =\frac{1-\mathrm{e}^{2 \mathrm{i}\left(\phi_{x}-\phi_{x-b}\right)}}{2 \pi^{2} b^{2}} \\
& =\frac{x b^{*}-x^{*} b}{2 \pi^{2}|b|^{2} x^{*}(x-b)}, \quad\left(x \equiv x^{1}+\mathrm{i} x^{2} \in \mathbb{C}\right) .
\end{aligned}
$$

[Equations (3.23)-(3.24) are proven in Appendix C.]

As is the case for the soft current (3.18), (3.22) is valid as it stands only if the initial $p_{1}$ direction is along the $z$ axis. However, for a generic $p_{1}$ direction, the amplitude in the Regge limit transforms in the same way as the soft one in Eq. (3.16), that is

$\mathcal{M}_{\text {Regge }}^{\left(\boldsymbol{\Theta}_{i}\right)}(\boldsymbol{b} ; E, \omega, \boldsymbol{\theta})=\mathrm{e}^{2 \mathrm{i}\left(\phi_{\theta-\boldsymbol{\Theta}_{i}}-\phi_{\boldsymbol{\theta}}\right)} \mathcal{M}_{\text {Regge }}\left(\boldsymbol{b} ; E, \omega, \boldsymbol{\theta}-\boldsymbol{\Theta}_{i}\right)$

(where $E \boldsymbol{\Theta}_{i}$ is the transverse part of the 4-momentum $p_{1}$ ), as directly proven in Appendix A.

To connect the small-angle (soft) and large-angle (Regge) regimes of the one graviton emission amplitude, it is convenient to rewrite Eq. (3.22) in terms of the (complex) variables $\theta=q / \omega$ and $\theta_{s}=q_{2} / E$ :

$$
\begin{aligned}
& \mathcal{M}_{\text {Regge }}(\boldsymbol{b} ; E, \omega, \boldsymbol{\theta}) \mid \\
& =\sqrt{\alpha_{G}} \frac{R}{\pi} \frac{E}{\omega} \int \frac{\mathrm{d}^{2} \boldsymbol{\theta}_{s}}{2 \pi|\theta|^{2}} \mathrm{e}^{\mathrm{i} E \boldsymbol{b} \cdot \boldsymbol{\theta}_{s}} \frac{1}{2} \frac{\theta \theta_{s}^{*}-\theta^{*} \theta_{s}}{\theta_{s}\left(\theta_{s}-\frac{\omega}{E} \theta\right)^{*}} .
\end{aligned}
$$

This expression differs from Eq. (3.8) by the replacement

$$
\frac{1}{\left|\theta_{s}\right|^{2}} \frac{\theta \theta_{s}^{*}-\theta^{*} \theta_{s}}{\theta\left(\theta-\theta_{s}\right)^{*}} \rightarrow \frac{1}{|\theta|^{2}} \frac{\theta \theta_{s}^{*}-\theta^{*} \theta_{s}}{\theta_{s}\left(\theta_{s}-\frac{\omega}{E} \theta\right)^{*}}
$$

in the integrand of Eq. (3.26). By inspection, we see the important point that Regge and soft evaluations agree in region (b), in which the condition $|\theta| \gg\left|\theta_{s}\right| \gg \frac{\omega}{E}|\theta|$ ensures that we are in the large-angle regime in the lhs with negligible internal insertions in the rhs, while Eq. (3.26) remains the only acceptable expression in region (c), where $\left|\theta_{s}\right|<\frac{\omega}{E}|\theta|$.

Therefore, in order to get a reliable emission amplitude holding in all regions $(\mathrm{a} \cup \mathrm{b} \cup \mathrm{c})$, we have to match the soft with the Regge evaluations. We start from the Fourier transform in Eq. (3.8) and we then add the difference of
Regge and soft evaluations of Eq. (3.27) in region (c) and in part of region (b), the border being parametrized by the cutoff $\Delta_{c}>1$ (see Fig. 3). Such difference has the form

$$
\begin{aligned}
\Delta \mathcal{M} \equiv & {\left[\mathcal{M}_{\text {Regge }}-\mathcal{M}_{\text {soft }}\right]_{\mathrm{c} \cup(\text { part of b) }} } \\
= & \sqrt{\alpha_{G}} \frac{R}{\pi} \frac{E}{\omega} \frac{1}{2} \int_{0}^{\Delta_{c} \frac{\omega}{E} \theta \mid} \frac{\mathrm{d}^{2} \boldsymbol{\theta}_{s}}{2 \pi\left|\theta_{s}\right|^{2}} \frac{\theta \theta_{s}^{*}-\theta^{*} \theta_{s}}{|\theta|^{2}} \\
& \times\left[\frac{1}{1-\frac{\omega}{E} \theta_{\theta_{s}^{*}}^{*}}-\frac{1}{1-\frac{\theta_{s}^{*}}{\theta^{*}}}\right] \mathrm{e}^{\mathrm{i} E \boldsymbol{b} \cdot \boldsymbol{\theta}_{s}},
\end{aligned}
$$

where we require $\left|\frac{E \theta_{s}}{\omega \theta}\right|=\mathcal{O}(1)$, with

$$
\left|\frac{E \theta_{s}}{\omega \theta}\right| \equiv\left|\frac{\tilde{\theta}_{s}}{\theta}\right|<\Delta_{c}, \quad\left|\frac{\theta_{s}}{\theta}\right|<\frac{\omega}{E} \Delta_{c} \ll 1
$$

so that we get the expression

$\Delta \mathcal{M} \simeq \sqrt{\alpha_{G}} \frac{R}{\pi} \frac{1}{2} \int_{0}^{|\theta| \Delta_{c}} \frac{\mathrm{d}^{2} \tilde{\boldsymbol{\theta}}_{s}}{2 \pi} \frac{\theta \tilde{\theta}_{s}^{*}-\theta^{*} \tilde{\theta}_{s}}{|\theta|^{2}\left|\tilde{\theta}_{s}\right|^{2}}\left(\frac{1}{1-\frac{\theta^{*}}{\tilde{\theta}_{s}^{*}}}-1\right) \mathrm{e}^{\mathrm{i} \omega b \cdot \tilde{\theta}_{s}}$

If we then choose $1 \ll \Delta_{c} \ll E / \omega$ the result (3.30) is weakly cutoff dependent and, in the $\Delta_{c} \rightarrow \infty$ limit, is formally equal to the negative of the Fourier transform of the soft amplitude on the whole phase space, rescaled at $E=\omega$ or, in other words,

$$
\begin{aligned}
\Delta \mathcal{M}^{\stackrel{\Delta_{c} \gg 1}{\rightarrow}}-\sqrt{\alpha_{G}} \frac{R}{\pi} \int \frac{\mathrm{d}^{2} \tilde{\boldsymbol{\theta}}_{s}}{2 \pi\left|\tilde{\theta}_{s}\right|^{2}} \mathrm{e}^{\mathrm{i} \omega \boldsymbol{b} \cdot \tilde{\boldsymbol{\theta}}_{s}} \frac{1}{2} \frac{\theta \tilde{\theta}_{s}^{*}-\theta^{*} \tilde{\theta}_{s}}{\theta\left(\theta-\tilde{\theta}_{s}\right)^{*}} \\
=-\mathcal{M}_{\text {soft }}(\boldsymbol{b} ; \omega, \omega, \boldsymbol{\theta}) .
\end{aligned}
$$

By then using Eq. (3.8) we obtain the explicit form of the matched amplitude (with explicit $\hbar$ dependence)

$$
\begin{aligned}
\mathcal{M}_{\text {matched }} \simeq & \mathcal{M}_{\text {soft }}(\boldsymbol{b} ; E, \omega, \theta)-\mathcal{M}_{\text {soft }}(\boldsymbol{b} ; \hbar \omega, \omega, \theta) \\
= & \sqrt{\alpha_{G}} \frac{R}{\pi} \int \frac{\mathrm{d}^{2} \boldsymbol{\theta}_{s}}{2 \pi \boldsymbol{\theta}_{s}^{2}}\left(\mathrm{e}^{\mathrm{i} \frac{E}{\hbar} b \cdot \boldsymbol{\theta}_{s}} \frac{E}{\hbar \omega}-\mathrm{e}^{\mathrm{i} \omega \boldsymbol{b} \cdot \boldsymbol{\theta}_{s}}\right) \\
& \times \frac{1}{2}\left(\mathrm{e}^{2 \mathrm{i}\left(\phi_{\theta-\theta_{s}}-\phi_{\theta}\right)}-1\right) \\
= & \sqrt{\alpha_{G}} \frac{R}{\pi} \mathrm{e}^{-2 \mathrm{i} \phi_{\theta}} \int \frac{\mathrm{d}^{2} z}{2 \pi z^{* 2}} \mathrm{e}^{\mathrm{i} b \omega z \cdot \boldsymbol{\theta}} \\
& \times\left(\frac{E}{\hbar \omega} \log \left|\hat{\boldsymbol{b}}-\frac{\hbar \omega}{E} z\right|-\log |\hat{\boldsymbol{b}}-z|\right),
\end{aligned}
$$

where we have used the $z$ representation of the helicity phases (3.6), by rescaling the $z$ variable in the first term.

The final result of Eq. (3.32) — derived on the basis of the soft-insertion formulas-is expressed in terms of the ( $\omega$-dependent) soft field 


$$
\begin{aligned}
h_{s}(\omega, z) & \equiv \frac{1}{\pi^{2} z^{* 2}}\left(\frac{E}{\hbar \omega} \log \left|\hat{\boldsymbol{b}}-\frac{\hbar \omega}{E} z\right|-\log |\hat{\boldsymbol{b}}-\boldsymbol{z}|\right) \\
& \equiv-\frac{\Phi_{R}(\omega, \boldsymbol{z})}{\pi^{2} z^{* 2}}
\end{aligned}
$$

in which the function $\Phi_{R}$ turns out to be useful for the treatment of rescattering, too (Sec. IV B). Furthermore, for relatively large angles $\left[\theta \gg \theta_{m} \sim \hbar /(E b)\right]$, Eq. (3.32) involves values of $\hbar \omega|z| / E \lesssim \theta_{m} / \theta$ which are uniformly small, and the expressions (3.33) can be replaced by their $\omega \rightarrow 0$ limits

$h_{s}(z)=-\frac{\Phi(z)}{\pi^{2} z^{* 2}}, \quad \Phi(z) \equiv \hat{\boldsymbol{b}} \cdot z+\log |\hat{\boldsymbol{b}}-z|$.

The latter quantities have a classical meaning, $h_{s}(z)$ as a metric component (Sec. III D) and $\Phi(z)$ as a modulation function in the classical treatment of Ref. [31]. As a consequence, Eq. (3.32) takes the simpler form

$\mathcal{M}_{\text {matched }} \equiv \mathcal{M}=\sqrt{\alpha_{G}} \frac{R}{2} \mathrm{e}^{-2 \mathrm{i} \phi_{\theta}} \int \mathrm{d}^{2} z \mathrm{e}^{\mathrm{i} b \omega z \cdot \theta} h_{s}(z)$

that will be mostly used in the following. Replacing $h_{s}(z)$ by its $\omega$-dependent form is needed if we want to treat the very-small-angle region and some quantum corrections also.

From Eq. (3.35) we can see directly how the matching works. In fact, due to Eq. (3.32), the linear term (the log term) in Eq. (3.34) is in correspondence with external (internal) insertions of the emission current. In region (a), where $z$ is pretty large, the linear term dominates and provides directly the soft limit. In region (b), the basic soft behavior (3.14) is reproduced but, with increasing values of $b|\boldsymbol{q}|$, it is actually canceled by internal insertions in region (c), because in the small $|z|$ limit the function $\Phi(z)$ is of order $\sim \mathcal{O}\left(|z|^{2}\right)$. This is confirmed by the Regge representation (3.23) which shows, by inspection, a $1 /(b|\boldsymbol{q}|)^{2}$ behavior for $b|\boldsymbol{q}| \gg 1$.

To summarize, our matched amplitude (3.35), which, by construction, should be identical to the Regge one of Eq. (3.22) in region (c), is also a nice interpolation in $(b \cup c)$ and part of (a) with $|\boldsymbol{\theta}|>\theta_{m} .{ }^{3}$ For this reason we call Eq. (3.35) [Eq. (3.22)] the soft-based (Regge-based) representation of the same unified amplitude. Their identity can be directly proven by the equation

$$
\int \mathrm{d}^{2} z\left[\mathrm{e}^{-2 \mathrm{i} \phi_{\boldsymbol{\theta}}} h_{s}(z)-b^{2} h(\boldsymbol{b}, b \boldsymbol{z})\right] \mathrm{e}^{\mathrm{i} b \omega z \cdot \boldsymbol{\theta}}=0,
$$

\footnotetext{
${ }^{3}$ The moderate-angle restriction becomes unimportant when the resummation of Sec. IV extends the collinear region up to $\Theta_{s} \sim R / b \gg \theta_{m}$.
}

which can be explicitly checked by switching to $z, z^{*}$ variables and integrating by parts. Equation (3.36) is in turn a direct consequence of the differential identity

$$
\frac{\partial}{\partial z} h_{s}-\frac{\partial}{\partial z^{*}} b^{2} h=0
$$

that is related in Sec. III D to a transversality condition of the radiative metric tensor.

Our unified soft-Regge amplitude $\mathcal{M}$ has then, for a generic $\boldsymbol{\Theta}_{i}$, the form

$$
\begin{aligned}
\mathcal{M}^{\left(\boldsymbol{\Theta}_{i}\right)}(\boldsymbol{b}, \boldsymbol{\theta}) & =\sqrt{\alpha_{G}} \frac{R}{2} \mathrm{e}^{-2 \mathrm{i}\left(\phi_{\boldsymbol{\theta}}-\phi_{\boldsymbol{\theta}-\boldsymbol{\Theta}_{i}}\right)} \int \mathrm{d}^{2} \boldsymbol{x} \mathrm{e}^{\mathrm{i} \omega \boldsymbol{x} \cdot\left(\boldsymbol{\theta}-\boldsymbol{\Theta}_{i}\right)} h(\boldsymbol{b}, \boldsymbol{x}) \\
& =\sqrt{\alpha_{G}} \frac{R}{2} \mathrm{e}^{-2 \mathrm{i} \phi_{\theta}} \int \mathrm{d}^{2} z \mathrm{e}^{\mathrm{i} b \omega z \cdot\left(\boldsymbol{\theta}-\boldsymbol{\Theta}_{i}\right)} h_{s}(z),
\end{aligned}
$$

where the Regge-based (soft-based) representation is used in the first (second) line. Equations (3.32)-(3.33) provide an improved small-angle description and some quantum corrections.

\section{Radiative metric tensor}

To complete the picture of single-exchange radiation, we recall the parallel calculation of radiative corrections to the metric fields and to the effective action [7,13]. At first subleading level this amounts to calculating the H-diagram fields $\delta h$ and $\delta a$ [Figs. 6(b) and 6(c)] occurring in the metric. By leaving aside time delays [9] we obtain [13]

$$
\begin{aligned}
\mathrm{d} s^{2}-\eta_{\mu \nu} \mathrm{d} x^{\mu} \mathrm{d} x^{\nu}= & 2 \pi R\left[a(\boldsymbol{x}) \delta\left(x^{-}\right) \mathrm{d} x^{-2}+\bar{a}(\boldsymbol{x}) \delta\left(x^{+}\right) \mathrm{d} x^{+2}\right] \\
& +2(\pi R)^{2} \operatorname{Re}\left[\left(\hat{\epsilon}_{\mu \nu}^{T T}-\mathrm{i} \hat{\epsilon}_{\mu \nu}^{L T}\right) h(\boldsymbol{x}) \Theta\left(x^{+} x^{-}\right)\right] \\
& \times \mathrm{d} x^{\mu} \mathrm{d} x^{\nu},
\end{aligned}
$$

where, starting from $a_{0}$ in Eq. (2.8) we expand the profile function $a(x)$, to first order in $R^{2} / b^{2}$, and the field $h(x)$ in the form (Fig. 6)

$$
\begin{gathered}
a(\boldsymbol{x})=a^{(0)}+\frac{R^{2}}{b^{2}} a^{(1)}+\ldots, \\
\bar{a}(\boldsymbol{x})=a(\boldsymbol{b}-\boldsymbol{x}), \quad(\boldsymbol{x}=b \boldsymbol{z}) \\
|\partial|^{2} a^{(1)}=\frac{1}{\pi} \frac{1}{|z|^{4}} 2 \Phi(z), \\
h(\boldsymbol{x})=\frac{x-x^{*}}{2 \pi^{2} b x^{*}(x-b)},
\end{gathered}
$$

and $\hat{\epsilon}_{\mu \nu}$ are polarization tensor operators of the form, for instance, ${ }^{4}$

\footnotetext{
${ }^{4}$ With this prescription, the metric tensor (3.39) satisfies the transversality condition $\partial^{\mu} h_{\mu \nu}=0$. The polarization tensors differ from those of [13] by a factor of $1 / 2$.
} 
$2 \hat{\epsilon}_{i j}^{T T}=\varepsilon_{i l} \varepsilon_{j m} \frac{\nabla_{l} \nabla_{m}}{|\nabla|^{2}}=\delta_{i j}-\frac{\nabla_{i} \nabla_{j}}{|\nabla|^{2}}$,

$2 \hat{\epsilon}_{++}^{T T}=-\frac{\partial_{+}}{4 \partial_{-}}, \quad 2 \hat{\epsilon}_{--}^{T T}=-\frac{\partial_{-}}{4 \partial_{+}}, \quad 2 \hat{\epsilon}_{+-}^{T T}=\frac{1}{4}$

with similar ones of the $L T$ polarization.

Such results follow from a shock-wave solution of the effective-action equations of motion which expresses all metric components in terms of the basic scalar field $h(\boldsymbol{x}) \equiv 4|\partial|^{2} \phi(\boldsymbol{x})$, where the explicit, single-valued form of $\phi(x)$, not to be confused with the modulating function $\Phi(z)$, was found in [7] and is given by the single-valued function $\left(x \equiv x^{1}+\mathrm{i} x^{2}=b z\right)$

$$
\begin{aligned}
\phi\left(x, x^{*}\right)= & \phi\left(b-x^{*}, b-x\right) \\
= & \frac{1}{8 \pi}\left[\log \frac{x^{*}}{b} \log \left(1-\frac{x}{b}\right)+\frac{x}{b} \log \frac{x^{*}}{b}\right. \\
& \left.+\left(1-\frac{x^{*}}{b}\right) \log \left(1-\frac{x}{b}\right)+f\left(\frac{x}{b}\right)+f\left(1-\frac{x^{*}}{b}\right)\right],
\end{aligned}
$$

where $f^{\prime}(x)=\frac{x}{x-1} \log x$ is devised so as to cancel the discontinuities at the $x=0$ and $x=b$ singularities.

From Eqs. (3.43) and (3.39), we obtain, in particular, the transverse plane metric components

$$
\begin{gathered}
h_{x x^{*}}=|\partial|^{2} \operatorname{Re} \phi=\frac{1}{8}(h(x)+h(b-x))=\frac{1}{4} \operatorname{Re} h(x), \\
h_{x^{*} x^{*}}=-\partial^{* 2} \operatorname{Re} \phi=-\frac{1}{8}\left(h_{s}(z)+h_{s}(1-z)\right)=h_{x x}^{*},
\end{gathered}
$$

which are closely related to the fields $h$ and $h_{s}$ introduced previously, because of the derivatives

$$
\left\{\begin{array}{l}
4 \partial^{* 2} \phi=-\frac{1}{\pi^{2}} \frac{1}{z^{* 2}} \Phi(z)=h_{s}(z)=\frac{\partial^{*}}{\partial} h(x) \\
4 \partial^{2} \phi=-\frac{1}{\pi^{2}} \frac{1}{(1-z)^{2}} \Phi(\hat{\boldsymbol{b}}-z)=h_{s}^{*}(1-z)=\frac{\partial}{\partial^{*}} h(x) .
\end{array}\right.
$$

We note some important points. First, the $x \leftrightarrow b-x$ symmetry of the metric is realized in the emission amplitude by exchanging jet 1 [or $h_{s}(z)$ ] and jet 2 [or $h_{s}^{*}(1-z)$ ]. Furthermore, the relationships of $h_{s}(z)\left[h_{s}^{*}(1-z)\right]$ with $h(x)\left[h^{*}(b-x)\right]$ in jet 1 (jet 2), already given in Eq. (3.37) for jet 1 , express the transversality conditions of the metric components

$$
\partial^{*} h_{x x^{*}}+\partial h_{x^{*} x^{*}}=0 \quad\left(\partial h_{x x^{*}}+\partial^{*} h_{x x}=0\right)
$$

and are thus rooted in the spin-2 structure of the interaction. Furthermore, in parallel with the soft-based representation (3.35) for jet 1 , we have by (3.45) the corresponding representation of the same amplitude in jet 2

$$
\mathcal{M}=\sqrt{\alpha_{G}} \frac{R}{2} \mathrm{e}^{2 \mathrm{i} \phi_{\theta}} \int \mathrm{d}^{2} z \mathrm{e}^{\mathrm{i} b \omega z \cdot \theta} h_{s}^{*}(1-z) .
$$

Note however that, while $h(\boldsymbol{x}) \sim h_{x x^{*}}$ is a scalar, the soft fields $h_{s}(z)\left[h_{s}^{*}(1-z)\right]$ in jet 1 (jet 2) have $J_{z}=2(-2)$ for rotations in the transverse plane, and in fact the opposite phase is factorized in Eq. (3.35) [Eq. (3.47)] which is relevant for jet 1 (jet 2 ) and helicity $\lambda=-2$. In all cases the resulting amplitude will come out invariant for rotations around the $z$ axis.

Finally, by starting from the soft-based representations just mentioned at $\boldsymbol{\Theta}_{i}=0$ we can complete the symmetry $\boldsymbol{x} \rightarrow \boldsymbol{b}-\boldsymbol{x}$ by constructing the helicity amplitudes for both jets at $\boldsymbol{\Theta}_{i} \neq 0$, with the result

$$
\mathcal{M}_{\lambda}^{\left(-\boldsymbol{\Theta}_{i}\right)}\left(\boldsymbol{b} ;-q^{3},-\boldsymbol{q}\right)=\mathcal{M}_{-\lambda}^{\left(\boldsymbol{\Theta}_{i}\right)}\left(\boldsymbol{b} ; q^{3}, \boldsymbol{q}\right) \mathrm{e}^{-\mathrm{i} \boldsymbol{b} \cdot \boldsymbol{q}_{\perp}},
$$

which relates opposite helicities with opposite 3-momenta $\vec{q}$ as a function of $\boldsymbol{q}_{\perp}=\boldsymbol{q}-\omega \boldsymbol{\Theta}_{i}$, transverse to the $\boldsymbol{\Theta}_{i}$ direction. The factor $\mathrm{e}^{-\mathrm{i} \boldsymbol{b} \cdot \boldsymbol{q}_{\perp}}$ ensures the translation $\boldsymbol{x} \rightarrow \boldsymbol{b}-\boldsymbol{x}$. Equation (3.48) can be checked in a straightforward way by using the explicit helicity projections of Appendix A, and is anyway a consequence of the helicity transformation properties of the soft fields in either jet.

We then conclude that the radiative metric components of Eq. (3.39) (based on the shock-wave solution of [7]) are consistent with the present single-exchange soft-Regge amplitudes and actually explain the unifying relationships by a transversality condition of the metric tensor. We see however that taking into account the helicity transformation phases is essential for completing the calculation of graviton emission, and superimposing single-exchange terms all along eikonal scattering.

\section{FACTORIZATION AND RESUMMATION}

\section{A. $b$ factorization and matching}

So far we have considered the radiation associated to the single-graviton exchange contribution to the basic Planckian scattering process. But we know (Sec. II) that such a high-energy process is described by the eikonal resummation of a large number $\sim G s / \hbar$ of single hits, so that, for a given impact parameter value $b$, the scattering angle increases from $\theta_{m}=\hbar /(E b)$ to $\left|\theta_{s}\right|=$ $2(G s / \hbar)(\hbar / E b)=2 R / b$, the Einstein deflection angle. This fact considerably enlarges the quasicollinear region with respect to $\theta_{s}$, which might be an important source of energy loss by radiation, so as to endanger energy conservation [25] unless explicitly enforced.

We start noticing that, despite the enlargement of the quasicollinear region, the external-line insertion amplitude (3.5) stays unchanged, being only dependent on the overall momentum transfer $\boldsymbol{Q}=\boldsymbol{E} \boldsymbol{\theta}_{s}$ of the process. Therefore, the damping of the collinear region and the large-angle 
behavior are both built in Eq. (3.12) for any values of $\theta_{s}$, as shown by Eq. (3.13).

One may wonder to what extent—or for which $\omega$ values - the external-line insertion method is tenable. There are two types of internal-line insertions: those on the fast, nearly on-shell particle lines of the eikonal iteration, and those on the exchanged-graviton lines. We now argue that both kinds of internal insertions can be taken into account using the soft/Regge matching strategy described in Sec. III C.

The first thing to notice is that fast-particle insertions are in fact already implicitly included in the soft approximation (3.5). In fact, in a general $n$-exchange contribution to eikonal scattering for each pair of propagating lines there are two pairs of insertions, one with the mass shell on the right (final) and one on the left (initial), whose currents are nearly equal (to order $\hbar \omega / E$ ) and opposite in sign. Thus, the purely soft emission can be written in two equivalent ways, one as in Eq. (3.5) as a purely external line insertion, and the other as a sum of $n$ contributions, where the insertion-still of the form (3.5) - is made internally on the fast lines which surround the $i$ th exchanged graviton. Since in general these fast lines will have accumulated a nonnegligible transverse momentum, the $i$ th internal insertion will be of the rotated form (3.18), with $\boldsymbol{\Theta}_{i}=\sum_{j=1}^{i-1} \boldsymbol{\theta}_{j}$.

As for insertions on exchanged graviton lines, they are negligible if the emitted transverse momentum $\boldsymbol{q}=\hbar \omega \boldsymbol{\theta}$ is smaller than any of the exchanged graviton lines which, in the Regge limit, are all of order $\left\langle q_{i}\right\rangle \sim \hbar / b$, thus leading to the condition $b|q| \ll \hbar$. The latter is not surprising because precisely this parameter occurs in the subtraction term of Eq. (3.32) coming from the Regge estimate of region (c) for a single exchange. Fortunately this region-which is generally multidimensional for $n$ exchanges-is most significant when both exchanged and emitted momenta are of the same order $\hbar \omega \ll E$. For double graviton exchange (Fig. 7), for instance, this corresponds to two diagrams, and more generally it allows one to count $n$ diagrams per eikonal contribution in which we have to compute and add $\Delta \mathcal{M}$, i.e., the difference of Regge and soft amplitudes introduced in Sec. III C. Again, this has to be adjusted to take into account the direction of the fast legs, which is straightforward since the Regge and soft amplitudes acquire the same transformation phase.

In the end, this means that all internal-line insertionsfor fast particles and exchanged particles alike-can be accounted for by calculating $n$ diagrams for the eikonal contribution with $n$ exchanged gravitons, where the matched amplitude [Eqs. (3.32) and (3.35)] is inserted in turn in correspondence to the $i$ th exchanged graviton, adjusting for the local incidence angle $\boldsymbol{\Theta}_{i}$ as in Eq. (3.18).

We recall at this point the soft-based representation of the matched amplitude (3.32), which, after adjusting for the incidence angle, acquires the form

$$
\mathcal{M}^{\left(\boldsymbol{\Theta}_{i}\right)}=\sqrt{\alpha_{G}} \frac{R}{2} \mathrm{e}^{-2 \mathrm{i} \phi_{\theta}} \int \mathrm{d}^{2} z h_{s}(\boldsymbol{z}) \mathrm{e}^{\mathrm{i} b \omega z \cdot\left(\boldsymbol{\theta}-\boldsymbol{\Theta}_{i}\right)}
$$

and is thus simply proportional to the translated Fourier transform (F.T.) of the soft field $h_{s}$.

We then use this representation for each "active" contribution. For $n=2$ (Fig. 7), for instance, we have $\boldsymbol{q}_{s 1}=E \boldsymbol{\theta}_{1}$ emitting with initial angle $\boldsymbol{\Theta}_{1}=0$ and $\boldsymbol{q}_{s 2}=$ $E \boldsymbol{\theta}_{2}$ with $\boldsymbol{\Theta}_{2}=\boldsymbol{\theta}_{1}$, and we write, accordingly,

$$
\begin{aligned}
\frac{\mathrm{e}^{2 \mathrm{i} \phi_{\boldsymbol{\theta}}} \mathcal{M}^{(2)}}{\sqrt{\alpha_{G}} \frac{R}{2}} & =\frac{1}{2}\left[\int \mathrm{d}^{2} z h_{s}(z) \mathrm{e}^{\mathrm{i} b \omega z \cdot \boldsymbol{\theta}}\left(2 \mathrm{i} \alpha_{G}\right) \int \frac{\mathrm{d}^{2} \boldsymbol{\theta}_{2}}{2 \pi \boldsymbol{\theta}_{2}^{2}} \mathrm{e}^{\mathrm{i} \frac{E}{\hbar} b \cdot \boldsymbol{\theta}_{2}}\right. \\
& \left.+\left(2 \mathrm{i} \alpha_{G}\right) \int \frac{\mathrm{d}^{2} \boldsymbol{\theta}_{1}}{2 \pi \boldsymbol{\theta}_{1}^{2}} \mathrm{e}^{\mathrm{i} \frac{E}{\hbar} \boldsymbol{b} \cdot \boldsymbol{\theta}_{1}} \int \mathrm{d}^{2} z h_{s}(z) \mathrm{e}^{\mathrm{i} b \omega \boldsymbol{z} \cdot\left(\boldsymbol{\theta}-\boldsymbol{\theta}_{1}\right)}\right] \\
& =\frac{2 \mathrm{i}}{2} \int \mathrm{d}^{2} z h_{s}(z) \mathrm{e}^{\mathrm{i} b \omega z \cdot \boldsymbol{\theta}}\left[\delta_{0}(b)+\delta_{0}\left(\left|\boldsymbol{b}-\frac{\hbar \omega}{E} b \boldsymbol{z}\right|\right)\right] .
\end{aligned}
$$

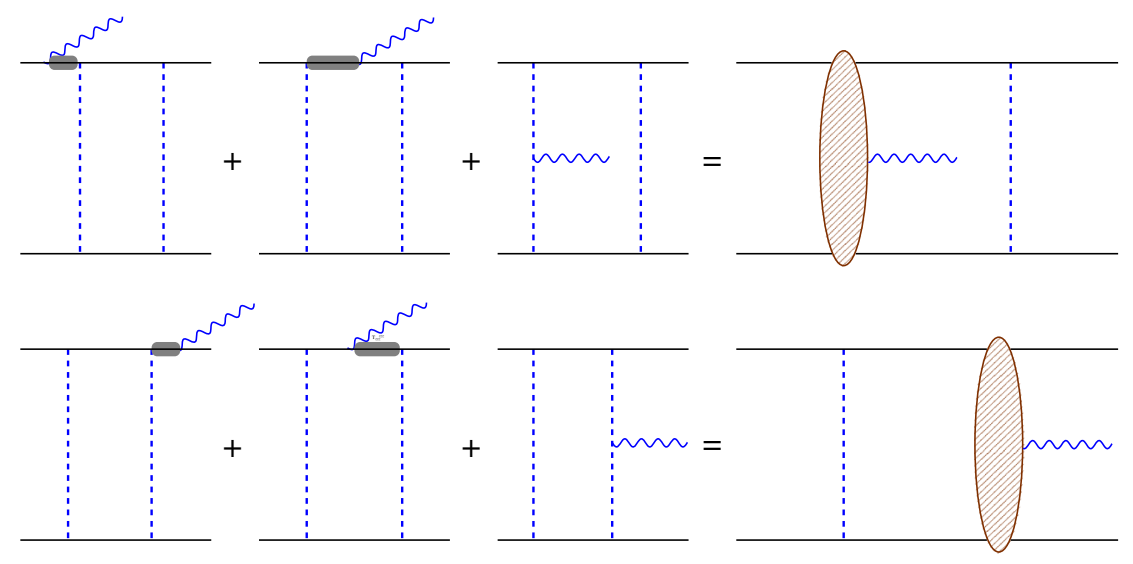

FIG. 7. Graviton insertions for double-exchange diagrams. External-line insertions are represented in the first column, internal-line insertions on the fast particles in the second column, and insertions on the exchanged graviton in the third column. Gray shadows around the fast particles denote off-shell propagation. Analogous insertion diagrams from the lower line are understood. The sum of each row amounts to inserting a matched emission amplitude (hatched brown blob) in place of a graviton propagator. 
We can see that the second active contribution, with nonzero incidence angle $\boldsymbol{\Theta}_{2}=\boldsymbol{\theta}_{1}$ has a translated $\boldsymbol{\theta}$ dependence, which amounts to factorizing an eikonal with $z$-dependent argument. This generalized factorization can be extended to the general case with $n>2$ exchanges, where however the $\boldsymbol{\theta}$ translation involves $\boldsymbol{\Theta}_{i}=\sum_{j=1}^{i-1} \boldsymbol{\theta}_{j}$, $i>2$, yielding higher powers of the eikonal with $z$-dependent argument. In formulas, we obtain, order by order,

$$
\begin{aligned}
\frac{\mathrm{e}^{2 \mathrm{i} \phi_{\theta}} \mathcal{M}_{\text {res }}}{\sqrt{\alpha_{G}} \frac{R}{2}}= & \int \mathrm{d}^{2} z h_{s}(z) \mathrm{e}^{\mathrm{i} b \omega z \cdot \boldsymbol{\theta}} \\
& \times\left\{1+\frac{2 \mathrm{i}}{2 !}\left[\delta_{0}(b)+\delta_{0}\left(\left|\boldsymbol{b}-\frac{\hbar \omega}{E} b z\right|\right)\right]\right. \\
& +\frac{(2 \mathrm{i})^{2}}{3 !}\left[\delta_{0}^{2}(b)+\delta_{0}(b) \delta_{0}\left(\left|\boldsymbol{b}-\frac{\hbar \omega}{E} b z\right|\right)\right. \\
& \left.\left.+\delta_{0}^{2}\left(\left|\boldsymbol{b}-\frac{\hbar \omega}{E} b z\right|\right)\right]+\ldots\right\} .
\end{aligned}
$$

Furthermore, the sum in square brackets is given by the expression

$$
\begin{aligned}
{[\ldots] } & =\frac{\mathrm{e}^{2 \mathrm{i} \delta_{0}(b)}-\mathrm{e}^{2 \mathrm{i} \delta_{0}\left(\left|\boldsymbol{b}-\frac{\hbar \omega}{E} b \boldsymbol{z}\right|\right)}}{2 \mathrm{i}\left[\delta_{0}(b)-\delta_{0}\left(\left|\boldsymbol{b}-\frac{\hbar \omega}{E} b \boldsymbol{z}\right|\right)\right]} \\
& =\mathrm{e}^{2 \mathrm{i} \delta_{0}(b)} \int_{0}^{1} \mathrm{~d} \xi \mathrm{e}^{-2 \mathrm{i} \xi \frac{G s}{\hbar} \log \left|\hat{b}-\frac{\hbar \omega}{E} z\right|},
\end{aligned}
$$

so that we finally get the factorized and resummed amplitude

$$
\begin{aligned}
\frac{\mathcal{M}_{\text {res }}}{\mathrm{e}^{2 \mathrm{i} \delta_{0}(b)}} & \equiv \mathfrak{M} \\
& =\sqrt{\alpha_{G}} \frac{R}{2} \mathrm{e}^{-2 \mathrm{i} \phi_{\theta}} \int \mathrm{d}^{2} z \mathrm{e}^{\mathrm{i} b \omega z \cdot \boldsymbol{\theta}} \int_{0}^{1} \mathrm{~d} \xi \mathrm{e}^{-2 \mathrm{i} \frac{G \xi \xi}{\hbar} \log \left|\hat{\boldsymbol{b}}-\frac{\hbar \omega}{E} z\right|} h_{s}(z) \\
& \simeq \sqrt{\alpha_{G}} \frac{R}{2} \mathrm{e}^{-2 \mathrm{i} \phi_{\theta}} \int \mathrm{d}^{2} z \int_{0}^{1} \mathrm{~d} \xi h_{s}(z) \mathrm{e}^{\mathrm{i} \omega b z \cdot\left(\boldsymbol{\theta}-\xi \boldsymbol{\Theta}_{s}(b)\right)}
\end{aligned}
$$

where we have expanded the logarithm in the exponent and neglected higher order terms in $\hbar / E b|\boldsymbol{\theta}|$. The latter can in principle be evaluated as quantum corrections to the basic formula of the last line.

A more symmetric expression for the resummed amplitude (4.5) is obtained in the Breit frame (also called brickwall frame), where the initial and final transverse momenta are equal and opposite [i.e., $\left.\pm \frac{1}{2} E \boldsymbol{\Theta}_{s}(\boldsymbol{b})\right]$. We can reach the Breit frame by rotating the system of $\frac{1}{2} \boldsymbol{\Theta}_{s}(\boldsymbol{b})$. According to Eq. (4.1), this amounts to translating $\boldsymbol{\theta} \rightarrow \boldsymbol{\theta}-\frac{1}{2} \boldsymbol{\Theta}_{s}(\boldsymbol{b})$, and at the end we obtain again the expression (4.5) but with $\xi$ integrated over the symmetric interval $[-1 / 2,1 / 2]$. In the following we often work in the Breit frame.
It is important to note that the $z$ dependence in Eq. (4.3) adds corrections to the naïve factorization of $\delta_{0}(b)$ [13] which for any given $n$ are of relative order $\hbar \omega\langle z\rangle / E \sim$ $\hbar /(b E|\boldsymbol{\theta}|)$ and thus may appear to be negligible in the region $|\boldsymbol{\theta}|>\theta_{m}$. However, this is not the case because of the counting factors of $\langle n\rangle \sim G s / \hbar$ occurring in Eq. (4.4), which promote such corrections to order $\left|\boldsymbol{\Theta}_{s}(\boldsymbol{b})\right| /|\theta|$ making them essential for the physics of the radiation problem at frequencies $\omega \sim R^{-1}$. The effect of such corrections can be summarized by the introduction of the resummed field in the Breit frame

$$
\begin{aligned}
h_{s}^{\mathrm{res}}(z) & \equiv h_{s}(z) \frac{\sin \left(\alpha_{G} \log \left|\hat{\boldsymbol{b}}-\frac{\hbar \omega}{E} z\right|\right)}{\alpha_{G} \log \left|\hat{\boldsymbol{b}}-\frac{\hbar \omega}{E} z\right|} \\
& \simeq h_{s}(z) \frac{\sin \omega R \boldsymbol{z} \cdot \hat{\boldsymbol{b}}}{\omega R z \cdot \hat{\boldsymbol{b}}} \quad\left(|z| \ll \frac{E}{\hbar \omega}\right)
\end{aligned}
$$

with its resummation factor which is directly $\omega R$ dependent in the moderate- $z$ form of the last line. Therefore, Eq. (4.5) can be summarized as

$$
\mathfrak{M}=\sqrt{\alpha_{G}} \frac{R}{2} \mathrm{e}^{-2 \mathrm{i} \phi_{\theta}} \int \mathrm{d}^{2} z h_{s}^{\text {res }}(z) \mathrm{e}^{\mathrm{i} b \omega z \cdot \boldsymbol{\theta}} .
$$

\section{B. Rescattering corrections and classical limit}

We have just seen that taking into account the sizeable incidence angles in multiple-exchange emission amplitudes provides important corrections to the naïve resummation formula which involve the effective coupling $\omega R=\frac{G s}{\hbar} \frac{\hbar \omega}{E}$ and are thus essential for $\omega R \simeq \mathcal{O}(1)$. One may wonder, at this point, whether additional corrections of relative order $\hbar \omega / E$ may be similarly enhanced by multiplicity factors, thus yielding important effects as well.

We do not have a complete answer to that question. We think however that kinematical corrections affecting incidence angles at relative order $\hbar \omega / E$ (and occurring in the currents' projections, Appendix A) are unimportant because they actually affect the factorization procedure at relative order $\mathcal{O}(\hbar \omega / E)^{2}$, and are thus subleading. On the other hand, we argue that dynamical corrections due to rescattering of the emitted graviton are to be seriously considered, even though they are known [7] to be subleading for the calculation of the scattering amplitude of the fast particles themselves.

Indeed, consider for instance the contributions to the emission amplitude of the two graviton-exchange diagrams of Fig. 7. If the active exchange is \#2, we just have to replace $\delta_{0}(b)$ by $\delta_{0}\left(\left|\boldsymbol{b}-\frac{\hbar \omega}{E} b \boldsymbol{z}\right|\right)$ because of the nontrivial incidence angle. But if the active exchange is \#1, the next hit is a three-body one, which involves emitted graviton interactions also, as illustrated in Fig. 8 for an emitted graviton in jet 1 (top), rescattering with jet 2 (bottom). Therefore, the remaining $\delta_{0}$ which was left uncorrected in Eq. (4.2) should be corrected also, by replacing it by 


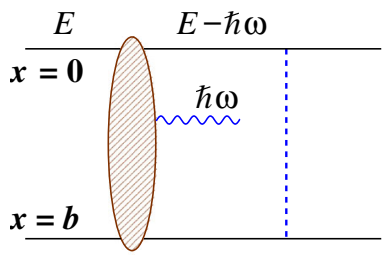

(a)

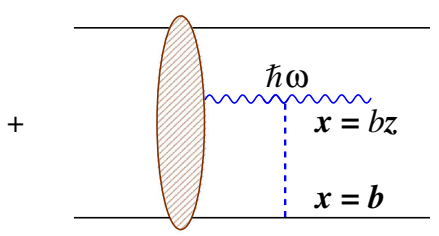

(b)
FIG. 8. Emission diagrams with subleading corrections. In (a) the eikonal scattering $\propto \delta(b)$ after graviton emission occurs with reduced energy $E \rightarrow E-\hbar \omega$. In (b) the graviton at $\boldsymbol{x}=b \boldsymbol{z}$ rescatters with the external particle at $x=b$ generating a term $\propto \hbar \omega \delta(|\boldsymbol{b}-b \boldsymbol{z}|)$.

$$
\begin{aligned}
\delta_{R}(\boldsymbol{b}, \boldsymbol{z}) & \equiv\left(1-\frac{\hbar \omega}{E}\right) \delta_{0}(b)+\frac{\hbar \omega}{E} \delta_{0}(|\boldsymbol{b}-b \boldsymbol{z}|) \\
& =\delta_{0}(b)-\omega R \log |\hat{\boldsymbol{b}}-\boldsymbol{z}|
\end{aligned}
$$

where we note that the fast-particle gravitational charge has been decreased by an energy conservation effect of order $\hbar \omega / E$, while the charge of the rescattering graviton is by itself of that order.

Since both replacements-due to incidence angles and rescattering alike - hold for any one of the single hits being considered [as in Eq. (4.3)] it follows that Eq. (4.4) should be replaced by

$$
[\ldots]=\frac{\mathrm{e}^{2 \mathrm{i}\left(\delta_{0}(b)-\omega R \log |\hat{b}-z|\right)}-\mathrm{e}^{2 \mathrm{i} \delta_{0}\left(\left|\boldsymbol{b} b \frac{\hbar \omega}{E} b z\right|\right)}}{2 \mathrm{i}\left[\delta_{0}(b)-\omega R \log |\hat{\boldsymbol{b}}-\boldsymbol{z}|-\delta_{0}\left(\left|\boldsymbol{b}-\frac{\hbar \omega}{E} b \boldsymbol{z}\right|\right)\right]}
$$

where we note the appearance, in the denominator, of the quantity $\Phi_{R}$ introduced in Eq. (3.33) multiplied by $2 i \omega R$. The first $\log$ in $\Phi_{R}$ is due to the incidence angle while the second one is due to rescattering. But since $-\Phi_{R}$ appears in the numerator if we upgrade $h_{s}(z)$ to $h_{s}(\omega, z)$ of Eq. (3.33), we simply get

$$
\begin{aligned}
\mathfrak{M}= & \sqrt{\alpha_{G}} \frac{R}{\pi} \mathrm{e}^{-2 \mathrm{i} \phi_{\theta}} \int \frac{\mathrm{d}^{2} z}{2 \pi} \frac{1}{z^{* 2}} \frac{\mathrm{e}^{\mathrm{i} \omega b z \cdot \theta}}{2 \mathrm{i} \omega R} \\
& \times\left(\mathrm{e}^{-2 \mathrm{i} \omega R \log |\hat{b}-z|}-\mathrm{e}^{-2 \mathrm{i} \omega R \frac{E}{\hbar \omega} \log \left|\hat{b}-\frac{\hbar \omega}{E} z\right|}\right),
\end{aligned}
$$

which is expressed as the algebraic sum of incidence angle and rescattering effects.

We thus see, by inspection, that since $\Phi_{R}(\omega, z) \rightarrow \Phi(z)$ in the small $\hbar \omega / E$ limit, Eq. (4.10) reproduces the classical amplitude of Ref. [31] with the proper normalization according to our conventions ${ }^{5}$ and helicity $\lambda=-2$,

\footnotetext{
${ }^{5}$ In order to carry out the precise comparison one should keep in mind that $R=4 E_{[32]}, \Phi=\frac{1}{8} \Phi_{[32]}$, and that scattering angles are defined with opposite sign conventions.
}

$\mathfrak{M}_{\text {class }}=\sqrt{\alpha_{G}} \frac{R}{\pi} \mathrm{e}^{-2 \mathrm{i} \phi_{\theta}} \int \frac{\mathrm{d}^{2} z}{2 \pi z^{* 2}} \frac{\mathrm{e}^{\mathrm{i} \omega b z \cdot\left(\boldsymbol{\theta}-\boldsymbol{\Theta}_{s}\right)}}{2 \mathrm{i} \omega R}\left(\mathrm{e}^{-2 \mathrm{i} \omega R \Phi}-1\right)$,

where $\boldsymbol{\Theta}_{s}(\boldsymbol{b})=-\frac{2 R}{b} \hat{\boldsymbol{b}}$ is, as usual, the fast-particle scattering angle.

We can also express the result (4.10) in a form similar to $(4.5)$ :

$$
\begin{aligned}
\mathfrak{M}= & \sqrt{\alpha_{G}} \frac{R}{2} \mathrm{e}^{-2 \mathrm{i} \phi_{\theta}} \int \mathrm{d}^{2} z h_{S}(\omega, z) \mathrm{e}^{\mathrm{i} b \omega z \cdot \theta} \\
& \times \int_{0}^{1} \mathrm{~d} \xi \mathrm{e}^{-2 \mathrm{i} \alpha_{G} \xi \log \left|\hat{b}-\frac{\hbar \omega}{E} z\right|-2 \mathrm{i} \omega R(1-\xi) \log |\hat{b}-z|},
\end{aligned}
$$

showing explicitly how, for each value of $\xi$, the incidence angle effect depends on the evolution up to an incidence angle $\boldsymbol{\xi} \boldsymbol{\Theta}_{s}$ while rescattering depends on the complementary interval $(1-\xi) \boldsymbol{\Theta}_{s}$ of incidence angles.

We can also say that the incidence angle dependence corresponds to the tilt in the fast-particle wavefront noted in [31], so that the rescattering counting is correctly reproduced by the simple overall subtraction in Eqs. (4.10)(4.11). Furthermore, the residual $\hbar \omega / E$ dependence of the improved form (4.10) produces quantum corrections to the classical formula (4.11). It is amazing that the same function $\Phi_{R}$ yields, on the one hand, the extension of the soft field to the small-angle part of region (a) and ensures, on the other hand, the rescattering corrections at quantum level.

In the following, we concentrate on the analysis of the result (4.5), which provides what we call the "geometrical" corrections due to scattering and emission with various incidence angles all over the eikonal evolution. The inclusion of rescattering corrections, leading to the classical result (4.11) and its quantum corrections (4.10), is deferred to a later work.

\section{The resummed amplitude and its regimes}

The amplitude $2 \mathfrak{M}(4.5)$ is directly normalized as the probability amplitude for the emission of a graviton in a scattering process occurring at impact parameter $b$. Its interpretation is that of a coherent average of the singleexchange amplitude over scattering angles $\xi \boldsymbol{\Theta}_{s}=-\xi \frac{2 R}{b} \hat{\boldsymbol{b}}$ ranging from zero to $\boldsymbol{\Theta}_{s}$.

The final result of our calculation in the geometrical approximation and in the Breit frame can also be expressed, more explicitly, in the form

$\mathfrak{M}=\sqrt{\alpha_{G}} \frac{R}{\pi} \mathrm{e}^{-2 \mathrm{i} \phi_{\theta}} \int \frac{\mathrm{d}^{2} z}{2 \pi z^{* 2}} \mathrm{e}^{\mathrm{i} b q \cdot z} \frac{\sin (\omega R x)}{\omega R x} \Phi(z)$,

where $\Phi(\boldsymbol{z})$ was defined in Eq. (3.34).

In order to understand the $\omega$ dependence of Eq. (4.13), it is convenient to rescale $z \rightarrow \omega R \tilde{z}$ so as to write 
$\mathfrak{M}=\sqrt{\alpha_{G}} \frac{R}{\pi} \mathrm{e}^{-2 \mathrm{i} \phi_{\theta}} \int \frac{\mathrm{d}^{2} \tilde{z}}{2 \pi \tilde{z}^{* 2}} \mathrm{e}^{\mathrm{i} \tilde{z} \cdot \frac{2 \theta}{\left|\boldsymbol{\theta}_{s}\right|} \frac{\sin \tilde{x}}{\tilde{x}}}\left(\frac{\tilde{x}}{\omega R}+\log \left|\hat{\boldsymbol{b}}-\frac{\tilde{z}}{\omega R}\right|\right)$.

We can see that there are basically two regimes:

(1) $\omega R \ll 1$. In this case $\Phi$ is dominated by the linear term, except if $\frac{|\tilde{z}|}{\omega R} \simeq \frac{1}{\omega|\boldsymbol{\theta}| b} \ll 1$, in which case $\Phi\left(\frac{\tilde{z}}{\omega R}\right) \simeq \frac{1}{2}\left(\frac{|\tilde{z}|}{\omega R}\right)^{2} \cos \left(2 \phi_{z}\right)$ which is very small. Therefore, we recover the soft limit in the form

$$
\begin{aligned}
2 \mathfrak{M} & \simeq \frac{\sqrt{\alpha_{G}}}{\pi \omega} \frac{1}{2 \mathrm{i}}\left[\mathrm{e}^{2 \mathrm{i}\left(\phi_{\boldsymbol{\theta}-\frac{1}{2} \boldsymbol{\Theta}_{s}}-\phi_{\boldsymbol{\theta}}\right)}-\mathrm{e}^{2 \mathrm{i}\left(\phi_{\boldsymbol{\theta}+\frac{1}{2} \boldsymbol{\Theta}_{s}}-\phi_{\boldsymbol{\theta}}\right)}\right] \Theta(\hbar-|\boldsymbol{q}| b) \\
= & \frac{\sqrt{\alpha_{G}}}{\pi \omega} \mathrm{e}^{\mathrm{i}\left(\phi_{\boldsymbol{\theta}+\frac{1}{2} \boldsymbol{\Theta}_{s}}+\phi_{\boldsymbol{\theta}-\frac{1}{2} \boldsymbol{\Theta}_{s}}-2 \phi_{\boldsymbol{\theta}}\right)}\left[\frac{\left|\boldsymbol{\Theta}_{s}\right| \sin \phi_{\boldsymbol{\theta}+\frac{1}{2} \boldsymbol{\Theta}_{s}, \boldsymbol{b}}}{\left|\boldsymbol{\theta}-\frac{1}{2} \boldsymbol{\Theta}_{s}\right|}\right] \\
& \times \Theta(\hbar-|\boldsymbol{q}| b),
\end{aligned}
$$

where we note the close relationship of $2 \mathfrak{M}$ with the soft insertion factor in Eq. (3.8), evaluated-in the Breit frame-at scattering angle $\boldsymbol{\Theta}_{s}(\boldsymbol{b})$. The second line yields, in square brackets, the singularity-free expression $\sin \phi_{\boldsymbol{\theta}-\frac{1}{2} \boldsymbol{\Theta}_{s}, \boldsymbol{\theta}+\frac{1}{2} \boldsymbol{\Theta}_{s}}$ and shows how the coupling $\left|\boldsymbol{\Theta}_{s}(\boldsymbol{b})\right|=2 R / b$ is recovered. Furthermore, the cutoff $|\boldsymbol{q}| b<\hbar$ argued on the basis of the $\Phi$-function behavior is consistent with the $1 /(|\boldsymbol{q}| b)^{2}$ behavior of the Regge form of the amplitude (3.23).

(2) $\omega R \gtrsim 1$. In this regime, the amplitude starts feeling the decoherence factor $\sim \frac{1}{\omega R}$ due to the $\xi$ average, which eventually dominates the large frequency spectrum of the energy-emission distribution (IV D) and establishes the key role of $R$. According to Eq. (4.14), resummation effects due to the $\frac{\sin \tilde{x}}{\tilde{x}}$ factor are kinematically small in the region $\left|\theta_{x}\right| \gg\left|\boldsymbol{\Theta}_{s}\right|$ because $x \sim \frac{\left|\boldsymbol{\Theta}_{s}\right|}{\left|\theta_{x}\right|} \ll 1$. Instead, in the region $\left|\theta_{x}\right| \lesssim$ $\left|\boldsymbol{\Theta}_{s}\right|$ they are important and tend to suppress the amplitude for $\omega R>1$. In order to see how, we anticipate from Eq. (4.24) the energy distribution formula of gravitational wave (GW) radiation

$$
\frac{\mathrm{d} E^{G W}}{\mathrm{~d} \omega \mathrm{d} \Omega}=\hbar|2 \omega \mathfrak{M}|^{2}
$$

so that it is instructive to look at the combination $2 \omega \mathfrak{M}$ in the limit $\omega R \gg 1$, in which $x \sim \frac{1}{\omega R}$ is supposed to be small, in order to avoid a higher power decrease.

Since we should have $b \omega \theta_{y} y \sim b \omega \theta_{x} x \sim \mathcal{O}(1)$, the condition $x \sim \frac{1}{\omega R}$ leads to a phase space in which $x \ll y \sim \mathcal{O}(1)$ and $\theta_{y} \sim \frac{1}{b \omega} \ll \theta_{x} \sim\left|\boldsymbol{\Theta}_{s}\right|=\frac{2 R}{b}$. In this region, in the Breit frame, we get the limit

$$
\begin{aligned}
2 \omega \mathfrak{M} \rightarrow & \sqrt{\alpha_{G}} \int_{-\infty}^{+\infty} \frac{\mathrm{d} \tilde{x}}{\pi} \mathrm{e}^{\mathrm{i} \frac{b}{R} \tilde{\theta} \theta_{x}} \frac{\sin \tilde{x}}{\tilde{x}} \\
& \times \int_{-\infty}^{+\infty} \frac{\mathrm{d} y}{2 \pi y^{2}} \log \left(1+y^{2}\right) \mathrm{e}^{\mathrm{i} b \omega \theta_{y} y},
\end{aligned}
$$

where we have set $\tilde{x}=\omega R x$ and approximated $\Phi(x, y) \rightarrow \Phi(0, y)$.

We thus get a simple, factorized amplitude which-by performing the remaining integralshas the explicit form

$2 \mathrm{i} \omega \mathfrak{M} \rightarrow \sqrt{\alpha_{G}} \int_{1}^{\infty} \frac{\mathrm{d} \eta}{\eta^{2}} \mathrm{e}^{-b \omega\left|\theta_{y}\right| \eta \Theta}\left(1-\frac{b}{R}\left|\theta_{x}\right|\right)$

in both the forward and backward hemispheres.

The above limiting amplitude is strongly collimated around $\left|\theta_{y}\right| \sim \frac{1}{b \omega} \ll \frac{1}{\omega R}$ (by which $\phi_{\theta}$ is very small), for any $\left|\theta_{x}\right|<\left|\boldsymbol{\Theta}_{s}\right| / 2$, that is in the $\xi$-averaging region. Furthermore, that distribution (confirmed numerically; see Fig. 11) comes from the transverse space region $x=0$, which becomes dominant at large $\omega R$ s.

In fact, we can easily calculate the contribution of (4.17) to the integrated distribution, that is

$$
\frac{\mathrm{d} E^{G W}}{\mathrm{~d} \omega}=G s \Theta_{s}^{2} \frac{4}{3}(1-\log 2) \frac{1}{\omega R},
$$

where the coefficient comes from the $\theta_{y}$ integral, in agreement with the dominant $\frac{1}{\omega R}$ behavior of the spectrum to be discussed next.

\section{Multigraviton emission and coherent-state operator}

So far, we have considered single-graviton emission in the whole angular range. However, the extension to many gravitons by keeping the leading terms in the eikonal sense is pretty easy. For one emitted graviton we have factorized in $b$ space one active (emitting) exchange out of $n$ in $n$ ways. Similarly for two gravitons we count $n(n-1)$ pairs of active exchanges emitting one graviton each, plus $n$ exchanges which emit two gravitons, and so on. The first ones are independent and provide an exponential series for the single emission amplitudes we have just resummed, the second ones yield correlated emission for a pair of gravitons, and so on.

Resumming the independent emissions yields multiple emission amplitudes which are factorized in terms of the single-emission ones calculated so far. Virtual corrections are then incorporated by exponentiating both creation $\left(a_{\lambda}^{\dagger}(\vec{q})\right)$ and destruction $\left(a_{\lambda}(\vec{q})\right)$ operators of definite helicity $\lambda$ (normalized to a wave-number $\delta$-function commutator $\left.\left[a_{\lambda}(\vec{q}), a_{\lambda^{\prime}}^{\dagger}\left(\vec{q}^{\prime}\right)\right]=\hbar^{3} \delta^{3}\left(\vec{q}-\vec{q}^{\prime}\right) \delta_{\lambda \lambda^{\prime}}\right)$, as follows, 
$\hat{S}=\mathrm{e}^{2 \mathrm{i} \delta} \exp \left\{\int \frac{\mathrm{d}^{3} q}{\hbar^{3} \sqrt{2 \omega}} 2 \mathrm{i}\left[\sum_{\lambda} \mathfrak{M}_{\boldsymbol{b}}^{(\lambda)}(\vec{q}) a_{\lambda}^{\dagger}(\vec{q})+\right.\right.$ h.c. $\left.]\right\}$,

where the helicity amplitude $\mathfrak{M}_{\boldsymbol{b}}^{(-)}(\vec{q})=\left[\mathfrak{M}_{\boldsymbol{b}}^{(+)}\left(-\boldsymbol{q}, q^{3}\right)\right]^{*}$ is provided by Eq. (4.5) with a proper identification of variables. Since operators associated with opposite helicities commute, the above coherent-state operator is Abelian (and thus satisfies the Block-Nordsieck theorem) but describes both helicities, not only the IR singular longitudinal polarization.

The $S$ matrix (4.20) is formally unitary because of the anti-Hermitian exponent, but needs a regularization because of the IR divergence mentioned before, due to the large distance behavior $\left|h_{s}(z)\right| \sim|h(z)| \sim|z|^{-1}$ of the relevant fields. Because of the virtual real-emission cancellation, the regularization parameter can be taken to be $\Delta \omega$, the experimental frequency resolution (assumed to be much smaller than $b^{-1}$ ), whose role will be further discussed in Sec. V. With that proviso, we can now provide the normal ordered form of Eq. (4.20) when acting on the initial state, which we identify as the graviton vacuum state $|0\rangle$, as follows:

$\mathrm{e}^{-2 \mathrm{i} \delta} \hat{S}|0\rangle=\sqrt{P_{0}} \prod_{\lambda} \exp \left\{2 \mathrm{i} \int_{\Delta \omega} \frac{\mathrm{d}^{3} q}{\hbar^{3} \sqrt{2 \omega}} \mathfrak{M}_{b}^{(\lambda)}(\vec{q}) a_{\lambda}^{\dagger}(\vec{q})\right\}|0\rangle$,

where

$$
P_{0}=\exp \left\{-2 \int_{\Delta \omega} \frac{\mathrm{d}^{3} q}{\hbar^{3} \omega} \sum_{\lambda}\left|\mathfrak{M}_{\boldsymbol{b}}^{(\lambda)}(\vec{q})\right|^{2}\right\}
$$

is the no-emission probability, coming from the $a, a^{\dagger}$ commutators.

Due to the factorized structure of Eq. (4.21), it is straightforward to provide the full generating functional of inclusive distributions

$\mathcal{G}\left[z_{\lambda}(\vec{q})\right]=\exp \left\{2 \int_{\Delta \omega} \frac{\mathrm{d}^{3} q}{\hbar^{3} \omega} \sum_{\lambda}\left|\mathfrak{M}_{b}^{(\lambda)}(\vec{q})\right|^{2}\left[z_{\lambda}(\vec{q})-1\right]\right\}$

as functional of the fugacity $z_{\lambda}(\vec{q})$.

In particular, the unpolarized energy emission distribution of gravitational waves in the solid angle $\Omega$ and its multiplicity density are given by

$$
\begin{aligned}
\frac{\mathrm{d} E^{\mathrm{GW}}}{\mathrm{d} \omega \mathrm{d} \Omega} & =\hbar \omega \frac{\mathrm{d} N}{\mathrm{~d} \omega \mathrm{d} \Omega}=\left.\hbar \omega^{2} \sum_{\lambda} \frac{\delta \mathcal{G}}{\delta z_{\lambda}(\vec{q})}\right|_{z_{\lambda}=1} \\
& =2 \omega^{2} \hbar \sum_{\lambda}\left|\mathfrak{M}_{\boldsymbol{b}}^{(\lambda)}(\vec{q})\right|^{2} .
\end{aligned}
$$

Both quantities are discussed in the next section.

\section{THE ANGULAR/FREQUENCY SPECTRUM}

\section{A. Energy emission and multiplicity distributions}

Starting from the soft/Regge emission amplitude in Eq. (4.13) we obtain, by Eq. (4.24), the multiplicity distribution in either jet $J(J=1,2, z \equiv x+\mathrm{i} y, x \equiv \boldsymbol{z} \cdot \hat{\boldsymbol{b}})$,

$$
\begin{aligned}
\left.\frac{\omega \mathrm{d} N}{\mathrm{~d} \omega \mathrm{d}^{2} \boldsymbol{\theta}}\right|_{J}= & \alpha_{G} \frac{(\omega R)^{2}}{2 \pi^{2}}\left[\left|\int \frac{\mathrm{d}^{2} z}{\pi z^{* 2}} \mathrm{e}^{\mathrm{i} b \omega z \cdot \theta} \frac{\sin \omega R x}{\omega R x} \Phi(z)\right|^{2}\right. \\
& +(\boldsymbol{\theta} \rightarrow-\boldsymbol{\theta})]_{J}
\end{aligned}
$$

where the helicity sum provides the additional $\boldsymbol{\theta} \rightarrow-\boldsymbol{\theta}$ contribution, equivalent to a factor of 2 after angular integration. It is convenient to look first at the qualitative properties of the frequency dependence integrated over angles, by distinguishing the two regimes pointed out before.

(i) $\omega R \ll 1$. This is the infrared singular region originally looked at by Weinberg. The angular integration in jet 1 involves the two-dimensional vector $\boldsymbol{q}=\hbar \omega \boldsymbol{\theta}$ and the amplitude is dominated by its leading form (4.15). If $b|\boldsymbol{q}| / \hbar \ll \omega R\left(|\boldsymbol{\theta}| \ll \Theta_{s}=2 R / b\right)$ the amplitude is $\phi$ dependent, but is independent of $|\boldsymbol{\theta}|$ because of the cancellation of the collinear singularities due to the helicity conservation zero, which has been extended to the whole region $|\boldsymbol{\theta}|<\Theta_{s}$ by our method. Therefore, the distribution acquires the form $\sim$ const $\frac{\mathrm{d}^{2} \boldsymbol{\theta}}{\Theta_{s}^{2}} \Theta\left(\Theta_{s}-|\boldsymbol{\theta}|\right)$ which effectively cuts off the $b \boldsymbol{q}$ integration at $b|\boldsymbol{q}| / \hbar \geq \omega R$.

If instead $\omega R<b|\boldsymbol{q}| / \hbar<b \omega$ we enter the intermediate angular region $\Theta_{s}<|\boldsymbol{\theta}|<1$ where (3.35) agrees with the basic form $\sim \frac{\sin \phi_{\theta}}{b|\boldsymbol{q}|}$ of (3.14) already noticed in [6], so that the integrated distribution is of the type

$$
\begin{aligned}
\frac{\omega \mathrm{d} N}{\mathrm{~d} \omega} & =\alpha_{G} \frac{2}{\pi} \Theta_{s}^{2}\left(\int_{R / b}^{1} \frac{\mathrm{d}^{2} \boldsymbol{\theta}}{\pi \boldsymbol{\theta}^{2}} \sin ^{2} \phi_{b \boldsymbol{q}}+\text { const }\right) \\
& =\alpha_{G} \frac{2}{\pi} \Theta_{s}^{2}\left(\log \frac{2}{\Theta_{s}}+\text { const }\right) .
\end{aligned}
$$

More precisely, by Eq. (4.15), we get for the energyemission distribution

$$
\begin{aligned}
\frac{\mathrm{d} E^{\mathrm{GW}}}{\mathrm{d} \omega} & \equiv \hbar \omega \frac{\mathrm{d} N}{\mathrm{~d} \omega} \\
& =\frac{G s}{\pi} \Theta_{s}^{2} 2 \int_{0}^{1}|\boldsymbol{\theta}| \mathrm{d}|\boldsymbol{\theta}| \int_{0}^{2 \pi} \frac{\mathrm{d} \phi}{2 \pi} \frac{\sin ^{2} \phi_{\theta b}}{\left|\boldsymbol{\theta}-\boldsymbol{\Theta}_{s}\right|^{2}} \Theta(1-b \omega|\boldsymbol{\theta}|) \\
& \simeq \frac{G s}{\pi} \Theta_{s}^{2}\left[2 \log \min \left(\frac{b}{R}, \frac{1}{\omega R}\right)+\text { const }\right]
\end{aligned}
$$

where we have changed variables $\boldsymbol{\theta}+\frac{1}{2} \boldsymbol{\Theta}_{s} \rightarrow \boldsymbol{\theta}$, integrated on both jets, and used the cutoff $b \omega|\boldsymbol{\theta}|<1$ due to the large $b|\boldsymbol{q}|$ suppression. 
We thus find that the typical infrared distribution $\mathrm{d} \omega / \omega$ is kept only in the tiny region $\omega<b^{-1}$, with a rapidity plateau in the range $|y|<Y_{s}=\log \left(2 / \Theta_{s}\right)=\log (b / R)$, much restricted with respect to the full rapidity $Y=$ $2 \log (E b / \hbar) \sim \log s$ available in the single H-diagram evaluation. On the other hand, the corresponding small$\omega$ number density $(G s / \pi \hbar) \Theta_{s}^{2}$ agrees with that used in [6] for the calculation of the two-loop eikonal and with the zero-frequency limit (ZFL) of [26,38].

(ii) $1<\omega R<(G s / \hbar)$. In this region we think it is tenable to assume the completeness of the $\boldsymbol{q}$ states, so that the spectrum, integrated over $\mathrm{d}^{2}(\omega \boldsymbol{\theta})$ of Eq. (5.1) and on both jets, is obtained by the Parseval identity in the form

$$
\begin{aligned}
\frac{\mathrm{d} E^{\mathrm{GW}}}{\mathrm{d} \omega} & =\hbar \omega \frac{\mathrm{d} N}{\mathrm{~d} \omega} \\
& =2 G s \frac{\Theta_{s}^{2}}{\pi} \int \frac{\mathrm{d}^{2} z}{\pi|z|^{4}}\left(\frac{\sin \omega R x}{\omega R x}\right)^{2}|\Phi(z)|^{2} .
\end{aligned}
$$

We note the typical IR behavior $\mathrm{d} \omega / \omega$ of the number density which is present in this formulation also, and we also note the less typical $\tilde{x} \equiv \omega R x$ dependence $\left(\frac{\sin \tilde{x}}{\tilde{x}}\right)^{2}$ due to the coherent average over initial directions (4.6), occurring in the Fourier transform of the resummed field (4.7), which essentially acts as a cutoff $\Theta(1-\omega R|x|)$. Its action is $\omega R$ dependent, cuts off large values of $|x|$ for $\omega R \ll 1$, and reduces the integration to small ones for $\omega R \gg 1$.

In particular, for $\omega R \gg 1$, the emitted-energy spectrum is considerably suppressed by our treatment of the collinear region, with respect to naïve $\mathrm{H}$-diagram expectations. We get in fact from Eq. (5.4) for the emitted-energy fraction

$$
\begin{aligned}
\frac{\mathrm{d} E^{\mathrm{GW}}}{\sqrt{s} \mathrm{~d} \omega}= & \frac{\hbar \omega}{\sqrt{s}} \frac{\mathrm{d} N}{\mathrm{~d} \omega} \\
= & R \Theta_{s}^{2} \frac{1}{\pi} \int \frac{\mathrm{d} x \mathrm{~d} y}{\pi\left(x^{2}+y^{2}\right)^{2}}\left(\frac{\sin (\omega R x)}{\omega R x}\right)^{2} \\
& \times\left(x+\frac{1}{2} \log \left[(1-x)^{2}+y^{2}\right]\right)^{2} .
\end{aligned}
$$

We see that the spectrum is decreasing like $1 /(\omega R)^{2}$ for any fixed value of $x$, in front of an integral which is linearly divergent for $x \rightarrow 0$. This means effectively a $1 /(\omega R)$ spectrum. More precisely by integrating the averaging factor in the small- $x$ region we get, for $\omega R \gg 1$, the factor

$$
\int_{|x|<1} \frac{\mathrm{d} x}{\pi}\left(\frac{\sin (\omega R x)}{\omega R x}\right)^{2} \simeq \int_{|x|<1} \frac{\mathrm{d} x}{\pi} \frac{\sin (2 \omega R x)}{\omega R x} \simeq \frac{1}{\omega R}
$$

in front of the coefficient

$$
\frac{1}{4} \int_{-\infty}^{+\infty} \frac{\mathrm{d} y}{y^{4}} \log ^{2}\left(1+y^{2}\right)=\frac{2}{3} \pi(1-\log 2),
$$

thus obtaining the asymptotic spectrum

$$
\frac{\mathrm{d} E^{\mathrm{GW}}}{\sqrt{s} \mathrm{~d} \omega} \simeq \frac{2}{3}(1-\log 2) R \Theta_{s}^{2} \frac{1}{\omega R} \quad(\omega R \gg 1) .
$$

Therefore, the total emitted energy fraction up to frequency $\omega_{M}$ is given by

$$
\frac{E^{\mathrm{GW}}}{\sqrt{s}}=\Theta_{s}(b)^{2}\left[\frac{2}{3}(1-\log 2) \log \left(\omega_{M} R\right)+\ldots\right]
$$

in agreement with the preliminary estimate (4.19), and is small, of order $\Theta_{s}^{2}$, up to a logarithm of $\omega_{M}$.

It is important to note that such results follow from Eq. (5.4) which is independent of $\hbar$, and should therefore have a direct classical interpretation. Indeed, the classical expression of [31] - which is here obtained by including rescattering corrections (Sec. IV B)—has by Eq. (4.11) the form

$$
\frac{\mathrm{d} E_{\text {class }}^{\mathrm{GW}}}{\mathrm{d} \omega}=2 G s \frac{\Theta_{s}^{2}}{\pi} \int \frac{\mathrm{d}^{2} z}{\pi|z|^{4}}\left(\frac{\sin \omega R \Phi(z)}{\omega R}\right)^{2},
$$

which differs from (5.4) because $\omega R \Phi(z)$ occurs in exponentiated form. At small $\omega R \ll 1$ the two results are essentially equivalent. On the other hand, the large $\omega R$ behavior of (5.10) is provided by the whole small $|z|$ region when $\Phi \simeq-\frac{1}{2}|z|^{2} \cos (2 \phi)$, to yield the result

$$
\begin{aligned}
\frac{\mathrm{d} E_{\text {class }}^{\mathrm{GW}}}{\mathrm{d} \omega} & \simeq \frac{\sqrt{s} \Theta_{s}^{2}}{2 \pi \omega} \int_{0}^{2 \pi} \frac{\mathrm{d} \phi^{\prime}}{2 \pi} \int_{0}^{\infty} \frac{\mathrm{d} \xi}{\xi^{2}}\left[1-\cos \left(\xi \cos \phi^{\prime}\right)\right] \\
& =\frac{\sqrt{s} \Theta_{s}^{2}}{2 \pi \omega} .
\end{aligned}
$$

The latter is in agreement with the $1 / \omega$ behavior of Eq. (5.8) but with a slightly different coefficient $(2 \pi)^{-1} \simeq$ 0.16 instead of $(2 / 3)(1-\log 2) \simeq 0.20$. We conclude that rescattering effects are somewhat important at large $\omega R$, but do not change the qualitative $1 / \omega R$ behavior of the spectrum.

A related important question is whether the emitted energy fraction (5.9) is limited by the quantum energy bound $\omega_{M}<E / \hbar$ only, or instead should be cut off at the purely classical level. In such a case we would expect that the $\omega R$ distribution is further suppressed by higher order contributions to the Riemann tensor, yielding e.g. a $(\omega R)^{-2}$ behavior, or higher. An argument in favor of the classical cutoff, advocated in Ref. [31], is detailed in Appendix D.

\section{B. Frequency and angular dependence}

In this section we present plots of the resummed amplitude and of the corresponding radiated energy distribution obtained by numerical evaluation. In this way, besides verifying the asymptotic behaviors derived in Secs. IV C and VA, we can visualize the shape of such quantities in the transition region $\omega R \sim 1$. Furthermore, we 
can study the angular distribution of radiation and notice very peculiar features.

Our first task is to rewrite the resummed amplitude $\mathfrak{M}$ given in Eq. (4.5) by means of a representation with the lowest number of integrals, having good convergence properties. It is possible to integrate the last line of Eq. (4.5) in $\mathrm{d}^{2} z$ and express the result in terms of the special function

$$
\mathcal{F}(z) \equiv \int_{0}^{\infty} \mathrm{d} \eta \mathrm{e}^{-\eta} \frac{\eta}{z+\eta}=1-z \mathrm{e}^{z} E_{1}(z)
$$

strictly related to the exponential integral (and to the incomplete gamma function) $E_{1}(z)=\Gamma(0, z)$. We are thus left with a compact one-dimensional integral over $\xi$,

$$
\begin{aligned}
\mathfrak{M} & =\sqrt{\alpha_{G}} \frac{R}{2 \pi} \mathrm{e}^{-2 \mathrm{i} \phi_{\theta}} \int_{\xi_{\text {min }}}^{\xi_{\max }} \mathrm{d} \xi \frac{w}{w^{*}} \mathrm{e}^{\mathrm{i} w^{*}} \operatorname{Im}\left[\frac{\mathrm{e}^{\mathrm{i} w}}{w} \mathcal{F}(-\mathrm{i} w)\right], \\
w & \equiv \omega R\left(\frac{\theta}{\Theta_{s}}-\xi\right),
\end{aligned}
$$

where $\left[\xi_{\min }, \xi_{\max }\right]$ is $[0,1]$ in the lab frame and $[-1 / 2,1 / 2]$ in the Breit frame. The singularity at $w=0$, i.e., $\xi=\theta / \Theta_{s}$, is harmless, being integrable. From the previous expression it is clear that, apart from the prefactor $\sqrt{\alpha_{G}} R, \mathfrak{M}$ depends only on $\omega R,\left|\theta / \Theta_{s}\right|$ and $\phi_{\theta}$.

\section{Energy spectrum}

Let us start by displaying the main features of the gravitational wave spectrum of Eq. (4.24) in the geometrical approximation of Eqs. (4.13) and (5.4). In Fig. 9(a) we plot (in $\log$ scale) the differential emitted energy with respect to $\omega R$ and $\left|\theta / \Theta_{s}\right|$ (i.e., after integration over the azimuthal angle $\phi$ ).

Figure 9(a) shows very clearly that the spectrum is dominated by a flat plateau (where kinematically accessible) whose shape can be easily explained as follows. The spectrum falls on the left $\left(\theta<\Theta_{s}\right)$ because of phase space and the absence of collinear singularities. It also falls on the right when $\omega R=b q \Theta_{s} / \theta>\Theta_{s} / \theta$, since then $b q>1$ [see Eq. (4.15)]. The last limitation (shaded region on the right) is due to the trivial kinematic bound $\theta<1$. As a result, for fixed $\omega R<1$ the length of plateau in $\log \theta$ is $\log (1 / \omega R)$ while it disappears completely for $\omega R>1$.

This is the reason why the spectrum in $\omega$ shown in Fig. 9(b) (obtained by a further integration over the polar angle $|\theta|$ and summing the contributions of the two hemispheres) shows two very distinct regimes:

(i) $\omega R \ll 1$. In this regime the amplitude is well approximated by Eq. (5.3). We see that the really infrared regime holds only in the tiny region $\omega<1 / b \Leftrightarrow \omega R<\Theta_{s}$, with a rapidity plateau up to $|y|<Y_{s} \equiv \log (b / R)$ [in Fig. 9(a) these are the deepest horizontal sections of the plateau which are limited on the right by the shaded region]. Such a rapidity plateau is much smaller than $Y=\log (E b / \hbar)$, the rapidity range available in the single H-diagram emission. Correspondingly, the energy spectrum is flat, as one can see on the leftmost part of Fig. 9(b) for the lines with nonvanishing $\Theta_{s}$. On the other hand, here the small- $\omega$ number density in rapidity, $(G s / \pi) \Theta_{s}^{2}$, agrees with the one used in [6] and with the ZFL of [26,38]. For larger values of $\omega R$, as already noted, the length of the horizontal sections of the plateau decreases; therefore we observe a logarithmic decrease of the energy spectrum for $\omega R \lesssim 1$.

(ii) $1<\omega R<\omega_{M} R$. This is the most interesting region of the spectrum, which in Fig. 9(b) exhibits the large $\omega R$ decrease $\sim 1 / \omega R$, in perfect agreement with Eq. (5.8). This feature originates from graviton emission all along the eikonal chain, summarized in the resummation factor (4.6), which contains the effective coupling $\omega R$ and yields the decoherence effect for large $\omega R$ values which is exhibited in Fig. 9(b).

It is important to note that curves for various values of $\Theta_{s}$ (and thus of $b$ ) yield different ZFLs, as expected, but then
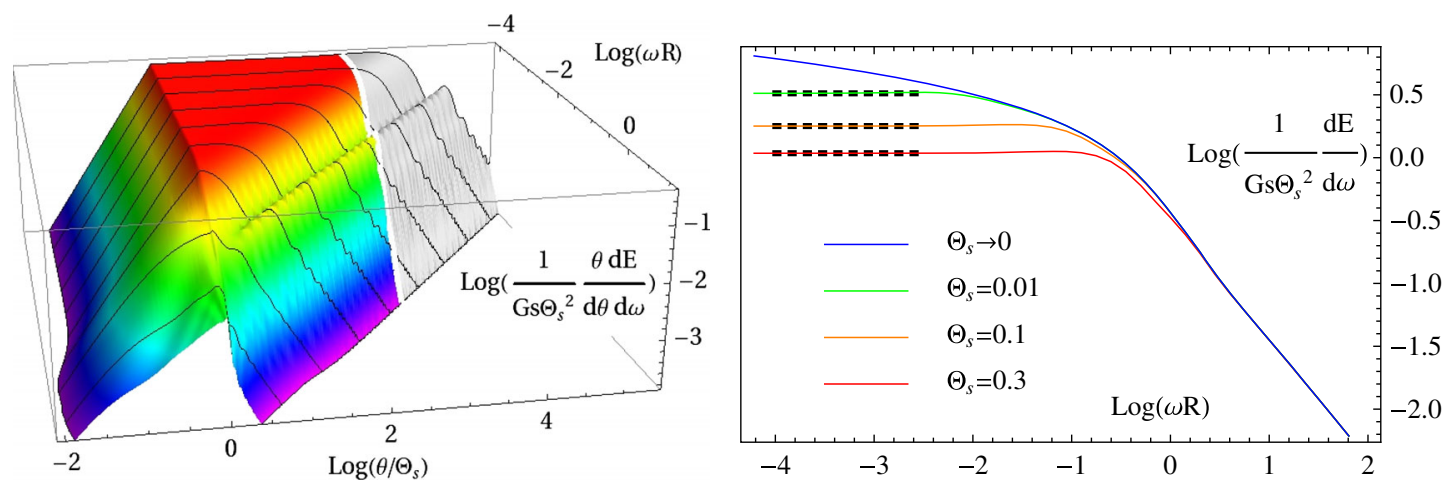

FIG. 9. (a) Left: Azimuthally integrated spectrum versus $\omega R$ and $\theta / \Theta_{s}$. The shaded region on the right is excluded by the kinematic bound $\theta<1$ (for the choice $\Theta_{s}=10^{-3}$ ). (b) Right: Frequency spectrum of gravitational radiation for various values of $\Theta_{s}$. For each $\Theta_{s}>0$ the ZFL value $\frac{2}{\pi} \log \left(1.65 / \Theta_{s}\right)$ is obtained (dashed lines). 


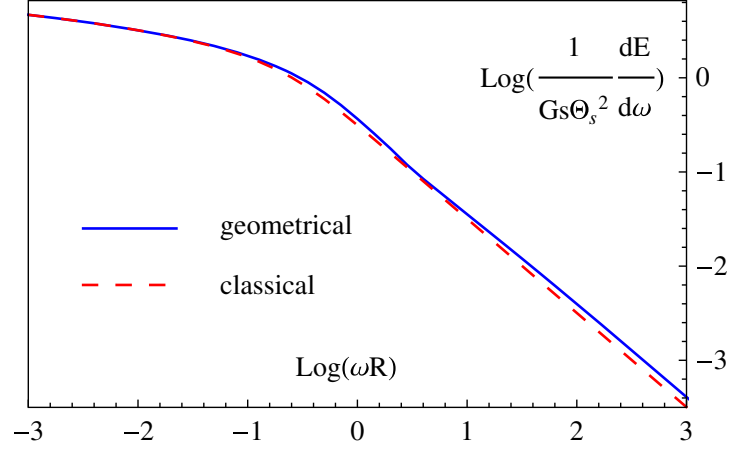

FIG. 10. Comparison of the universal limit of our energy spectrum in the geometrical approximation (solid blue) with the classical result of Ref. [31] (dashed red).

coalesce in a common curve for $\omega \gtrsim 1 / b$, the blue curve in Fig. 9(b), which is therefore universal and corresponds to Eq. (5.4).

It is interesting to compare (Fig. 10) the blue curve of Fig. 9(b) of our geometrical approach with the classical counterpart of Ref. [31] in Eq. (5.10). We can see that the agreement is pretty good, even if the difference starts being important at large $\omega R$ values, suggesting that rescattering
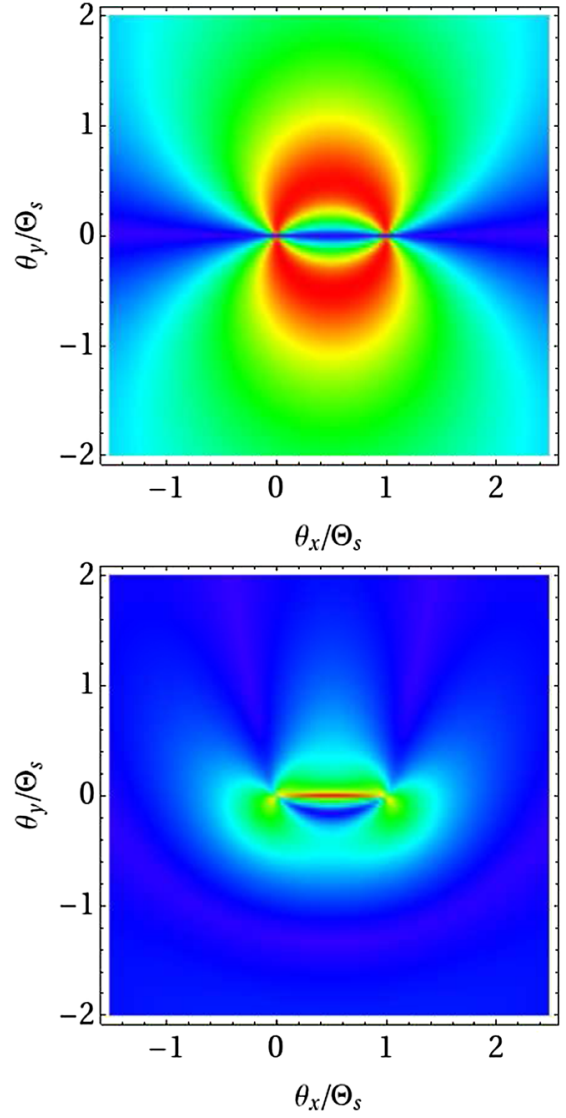

corrections [not included in Eq. (5.4)] and perhaps also quantum effects should be better evaluated in this region. For instance, the upper limit $\omega_{M}$ quoted here, which occurs in the total emitted-energy fraction (5.9), is provided in any case by phase space, i.e., by the quantum frequency $E / \hbar$. But it is likely that, as advocated in Ref. [31] and illustrated in Appendix D, the classical theory will provide by itself a physical cutoff, of order $\omega_{M} \sim R^{-1} \Theta_{s}^{-2}$.

\section{Angular distributions}

In order to have a picture of the full angular distribution of the radiation at various frequencies, in Fig. 11 we plot $|\mathfrak{M}|$ in the $\left\langle\theta_{x} / \Theta_{s}, \theta_{y} / \Theta_{s}\right\rangle$-plane for some values of $\omega R$; the maximum values of $|\mathfrak{M}|$ are attained in the red regions, while vanishing values of the amplitude are shown in blue.

We see that for $\omega R \ll 1$ the emission is symmetric with respect to the symmetry axes of the process, and is spread in a rather wide region around the particles' directions, in particular at $\left|\phi_{\theta}\right| \simeq \pi / 2$, in agreement with the $\sin \phi_{q}$ dependence of Eq. (4.15). Moving to larger values of $\omega R \sim 1$ the helicity amplitude shows an asymmetry with respect to the $x$ axis. The symmetry is restored by the symmetrical behavior of the amplitude with opposite
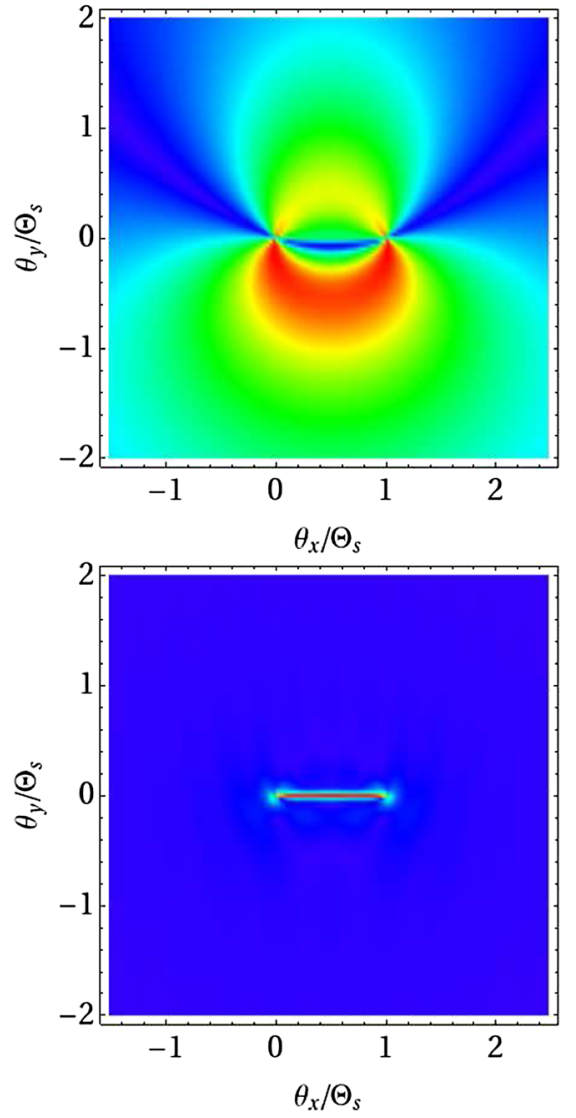

FIG. 11. Modulus of the resummed amplitude $|\mathfrak{M}|$ versus transverse direction $\boldsymbol{\theta}$, normalized to unit $\Theta_{s}$, for four values of $\omega R$. From left to right, top to bottom, $\omega R=0.001,0.125,1,8$. The increase of $|\mathfrak{M}|$ from 0 to its maximum (for each plot) is represented by colors ranging from blue to red. 
helicity. We note also a progressive shrinkage of the emission in the region close to the particles' directions. At large values of $\omega R \gg 1$ the effective support of the amplitude is just a thin strip around the interval $\theta \in\left[0, \Theta_{s}\right]$ whose width decreases as $1 /(\omega R)$, also in this case in agreement with the analytic estimate (4.18).

A more quantitative graphical representation of the azimuthal dependence of the amplitude is shown in Fig. 12, where we fix the polar angle $|\theta|=\frac{1}{2}\left|\Theta_{s}\right|$ and plot, for various values of $\omega R$, the amplitude versus $\phi_{\theta}$, rescaled by $\omega R$. At small $\omega R \ll 1$ (dotted green curve) we see the expected $\left|\sin \phi_{q}\right|$ behavior of Eq. (4.15) (it becomes exactly $\left|\sin \phi_{\theta}\right|$ for $|\theta|$ much larger or much smaller than $\left|\Theta_{s}\right|$ ). At intermediate $\omega R \sim 1$ (dashed blue curve) the asymmetry in $\phi_{\theta}$ is evident, and the enhancement around $\phi_{\theta} \simeq 0$ starts taking place. At large $\omega R \gg 1$ (solid red curve) the amplitude shows a narrow peak at $\phi_{\theta}=0$, whose width and height are inversely proportional to $\omega R$. At finite $\phi_{\theta} \neq 0$, the amplitude is more and more suppressed with increasing $\omega R$, according to Eq. (4.18).

On one hand, the presence of such a narrow peak [which becomes of constant height after multiplication by $\omega$; see Eq. (4.16)] explains the $(\omega R)^{-1}$ decrease of the radiated energy. On the other hand, it suggests that the radiation is sort of more and more confined along the trajectories of the fast particles with increasing $R \propto \sqrt{s}$, at fixed $b$ and $\omega$.

\section{Absorptive part and resummation effects}

We are now in a position to discuss the total emission multiplicity which is related to the imaginary part of the resummed scattering amplitude

$$
\langle N\rangle_{\Theta_{s}}=\int_{\Delta \omega} \frac{\mathrm{d} N}{\mathrm{~d} \omega} \mathrm{d} \omega=4 \operatorname{Im} \delta_{\text {res }}\left(\alpha_{G}, \Theta_{s}\right)
$$

and is also related to the no-emission probability $P_{0}=$ $\mathrm{e}^{-\langle N\rangle_{\Theta_{s}}}$ of Eq. (4.22). According to Eq. (4.6) its general expression is $\left(\Delta \omega=\mathcal{O}\left(b^{-1}\right)\right)$

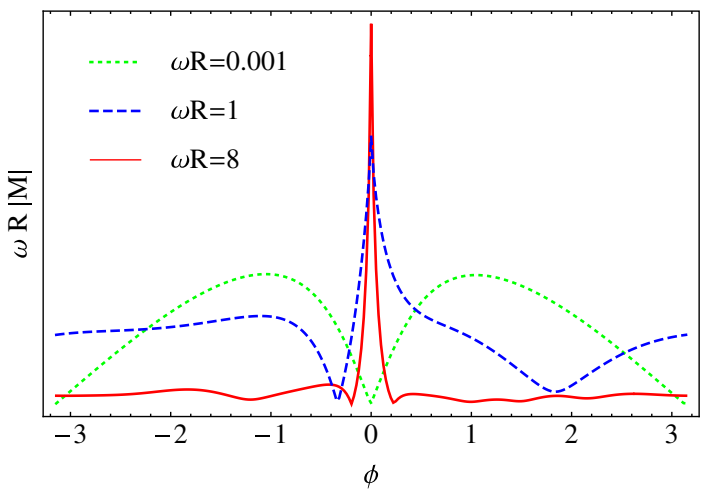

FIG. 12. Azimuthal dependence of the rescaled resummed amplitude $\omega R|\mathfrak{M}|$ (in arbitrary units) for $|\theta|=\frac{1}{2}\left|\Theta_{s}\right|$ and for three values of $\omega R$.

$$
\begin{aligned}
4 \operatorname{Im} \delta_{\text {res }}\left(\alpha_{G}, \Theta_{s}\right)= & \alpha_{G} \Theta_{s}^{2}(b) \frac{2}{\pi} \int_{\Delta \omega}^{E / \hbar} \frac{\mathrm{d} \omega}{\omega} \frac{\mathrm{d}^{2} z}{\pi|z|^{4}} \Phi^{2}(z) \\
& \times\left(\frac{\sin \alpha_{G} \log \left|\hat{\boldsymbol{b}}-\frac{\hbar \omega}{E} z\right|}{\alpha_{G} \log \left|\hat{\boldsymbol{b}}-\frac{\hbar \omega}{E} \boldsymbol{z}\right|}\right)^{2} \\
\stackrel{\alpha_{G} \gg 1}{\simeq} & \alpha_{G} \Theta_{s}^{2}(b) \frac{2}{\pi} \int_{\Delta \omega}^{E / \hbar} \frac{\mathrm{d} \omega}{\omega} \frac{\mathrm{d}^{2} z}{\pi|z|^{4}} \Phi^{2}(z) \\
& \times \frac{\sin ^{2} \omega R x}{(\omega R x)^{2}},
\end{aligned}
$$

where we have assumed $\Delta \omega=\mathcal{O}\left(b^{-1}\right)$ in order to have a reliable completeness of the $\boldsymbol{q}$ states.

In the first line of (5.15) we have made use of the general expression (4.6) of the resummed field valid for any $\alpha_{G}$, while in the second line we have considered the transPlanckian limit $\alpha_{G} \gg 1, \omega R$ fixed, which is the main interest of the present paper. Such two forms show very clearly that the estimate (5.15) for $\alpha_{G}$ moderate to small is substantially different from the one in the trans-Planckian limit. In the first regime the resummation factor is a power series in $\alpha_{G}$, starting from 1 for $\alpha_{G} \rightarrow 0$, limit in which (5.15) yields just the H-diagram result called $4 \operatorname{Im} \delta_{2}$ in [6]. As a consequence the $\omega$ values can go up to $\omega \sim E / \hbar$ yielding a relatively large emitted energy and showing the $\sim \log s$ dependence in rapidity used by Amati et al. [6] to hint at the real part of the amplitude from a dispersion relation. Furthermore, due to the logarithmic $\omega$ dependence of the resummation factor, the large- $\omega$ phase space is modified rather slowly by varying the $\alpha_{G}$ value, thus suggesting an intermediate regime where the real part could be calculated also.

On the other hand in the trans-Planckian regime $\left(\alpha_{G} \gg 1, \omega R\right.$ fixed) of the present paper, the second line of (5.15) shows that values of $\omega \gtrsim \mathcal{O}\left(R^{-1}\right)$ are substantially suppressed, thus leading to the reduced rapidity $Y_{s}=$ $2 \log (b / R)$ mentioned before, the subsequent resolution of the energy crisis, and the emergence of our Hawking-like radiation, which represents the main result of our investigation.

More precisely, in order to take into account arbitrarily small values of $\Delta \omega$ in the trans-Planckian case, we distinguish the soft and large-frequency contributions as in Sec. VA by writing, to logarithmic accuracy,

$$
\begin{aligned}
4 \operatorname{Im} \delta_{\text {res }}\left(\Theta_{s}\right)= & \frac{2 \alpha_{G}}{\pi} \Theta_{s}^{2}\left[\int_{\Delta \omega}^{1 / R} \frac{\mathrm{d} \omega}{\omega} \log \min \left(\frac{b}{R}, \frac{1}{\omega R}\right)\right. \\
& \left.+\int \frac{\mathrm{d}^{2} z}{\pi|z|^{4}} \Phi^{2}(z) \int_{|x|}^{\infty} \frac{\mathrm{d} \tilde{x} \sin ^{2} \tilde{x}}{\tilde{x}} \frac{\tilde{x}^{2}}{}\right] \\
\simeq & \frac{2 \alpha_{G}}{\pi} \Theta_{s}^{2}\left(\log \frac{b}{R} \log \frac{1}{\sqrt{R b} \Delta \omega}+Y_{>}\right) .
\end{aligned}
$$

We see that the large-frequency integral $\left(\omega>R^{-1}\right)$ yields just an $R$-independent constant rapidity $Y_{>} \simeq 0.56$, while 


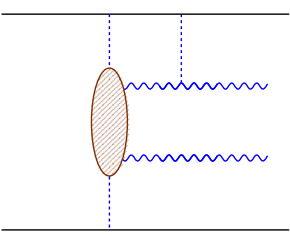

(a)

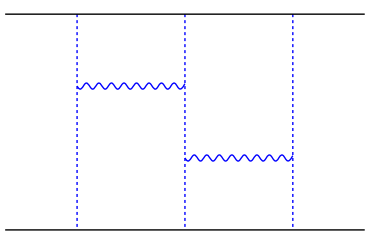

(b)

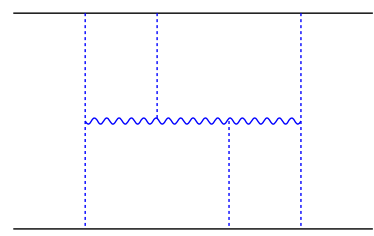

(c)

FIG. 13. Subleading effective diagrams representing (a) two-graviton correlations, (b) multi-H diagrams, and (c) rescattering corrections.

the soft one is determined by the reduced rapidity $Y_{s}=\log (b / R)$, with the physical consequences mentioned before. The phase space for the left part will eventually disappear with increasing $R$. This suggests that in the extreme energy (and large angle) limit, the total emission multiplicity will become just proportional to $\alpha_{G}$ with a coefficient of which Eq. (5.16) provides a provisional estimate.

\section{Towards large-angle resummation}

It is of obvious interest to try to extend the radiation treatment presented here to the extreme energy region $R \sim$ $b$ where the scattering angle $\Theta_{s}(b)$ becomes of order unity or larger, and a classical gravitational collapse may take place. By following the path led by [13] and mentioned before, we encounter two kinds of effects: (i) those due to the evaluation of the elastic eikonal function $\delta \sim \alpha_{G} f\left(R^{2} / b^{2}, l_{P}^{2} / b^{2}\right)$, which becomes a strong-coupling series showing perhaps some critical singularity at $b=R$, as in the reduced-action model [7]; and (ii) those arising from the $\xi$ averaging, that is the coherent sum of $\delta$ exchange emissions at the radiation level that we have just emphasized for $\delta=\delta_{0}$ as the origin of the key role of $R$ in the energy-emission spectrum.

By focusing on the second kind of effects, we may consider the first one as simply the source of some structure in $\delta(b)$ and in the related semiclassical trajectories [9] that will show up in the $\xi$ averaging also. Therefore, the new features of elastic scattering in the strong coupling regime for $R \sim b$ will provide new effects at the radiation level. A nice picture of the present situation is exhibited in Fig. 11(d), in which the frequencies $\omega>1 / R$ send a last signal before being suppressed. This supposedly essential message emphasizes the present span $2 R / b$ of the incidence angles $\xi \boldsymbol{\Theta}_{s}$, and the impact-parameter direction $\hat{b}$. Both parameters are expected to change with increasing $R$, because the semiclassical trajectory is likely to approach a quasibound shape and the question is how much that change will affect, by the $\xi$ averaging, the emerging radiation.

To provide an example, the impact-parameter direction $\hat{b}$ is expected to rotate by following the trajectory during time evolution, and thus it is possible (though not obvious) that the $\hat{x}$ and $\hat{y}$ directions will be mixed by the $\xi$ averaging. If that is the case, the $y$ variable will be, on the average, proportional to $x$ and, as a consequence, the modulation function $\Phi(z)$ will be small and of order $|z|^{2}$, thus acquiring a cylindrical symmetry and implying that the distribution (5.5) is of type $1 /(\omega R)^{2}$ [and not $1 /(\omega R)$ ]. That behavior would yield a faster suppression when approaching $R \sim b$ and would automatically provide a cutoff in the energy fraction (5.9).

It is of course important to establish whether such a sizeable change of the emerging radiation will really occur or not. This is nontrivial, however, because it requires a formal description of the $\xi$ averaging for higher orders in the $\delta$-exchange emission also. It would appear, though, that looking at the $\xi$ averaging by keeping the semiclassical trajectory standpoint may produce some changes, but smooth ones, with no real hints of information loss.

If we now switch to the full quantum level, it is clear that-besides modifying the $\xi$ averaging by single- $\delta$ emission-we have to modify multiple emissions also by introducing correlations in the coherent state (4.20) by the procedure of Sec. IV D. The simplest one concerns the two-graviton emission amplitude at order $G^{3} s^{2}$, an example of which is given in the diagram of Fig. 13(a), which introduces quadratic terms $\sim a^{2}, a^{\dagger 2}$ in the exponent of (4.20). At the same time such a diagram occurs in the corrections to the elastic eikonal exemplified in Figs. 13(b) and 13(c), which contain further powers of $s$ because of $s$-channel iteration, and are thus of second order in the effective coupling $R^{2} / b^{2}$. The calculation of 13(a) can be devised by following the lines of Sec. III in the various soft and Regge regions, so as to evaluate the correlation term.

Upgrading the present method to include the steps just described looks therefore within reach. It may shed light on the existence of a large $\omega_{M}$ cutoff, of possibly (higher order) classical origin or quantum mechanical one. Going much further however seems very hard, because treating both polarizations at higher orders of the effective-action expansion is a fully two-dimensional problem, unlike the reduced action model with one polarization in the axisymmetric case investigated in [13]. We nevertheless hope that, even just at the next order, the present approach may provide us with some global insight on the interplay of radiation and scattering in the strongcoupling regime. 


\section{SUMMARY AND PERSPECTIVES}

We have investigated, in this paper, the peculiar features of the graviton radiation associated to gravitational particle scattering at energies much larger than the Planck mass. That scattering, at small deflection angles, is described by a semiclassical $S$ matrix in $\boldsymbol{b}$ space, which exponentiates the eikonal function $\delta(\boldsymbol{b})$, of order $\alpha_{G} \equiv G s / \hbar \gg 1$ and expressed as a power series in $R^{2} / b^{2}, R \gg l_{P}$ being the gravitational radius of the system (Sec. II).

We find here that the ensuing radiation is expressed as a superposition of a large number $\sim \alpha_{G}$ of single-hit emission processes, each one being derived in a high-energy form which unifies the soft and Regge limits in the whole angular range (Sec. III). Combining the large emission number $(G s / \hbar \gg 1)$ with the relatively small emitted energy $(\hbar \omega / E \ll 1)$ produces in the emission amplitudes the effective coupling $\omega R$ which tunes the resulting spectrum on the inverse gravitational radius (Sec. IV). For that reason the emerging radiation is Hawking-like-that is with characteristic energies $\sim \hbar / R$ which decrease for increasing input energies, even in the small-deflection angle regime in which the $S$ matrix is explicitly unitary. In fact, as a consequence of coherence effects in the superposition just mentioned, our unified amplitudes are found to have a surprisingly simple interference pattern in $\omega R$, suppressing large frequencies $\omega \gg 1 / R$ and reducing the radiated energy fraction to order $\Theta_{s}^{2}$ (Sec. V).

Finally, we generalize the (quantum) factorization method of single-hit emissions to include rescatterings of the emitted graviton and to resum them (Sec. IV B). The ensuing emission amplitude neatly agrees with Ref. [31] in the classical limit and also includes the mentioned quantum effects in a simple and elegant way, thus calling for further investigation in the near future.

The ultimate goal of our thought experiment beyond the Planck scale is actually to reach large scattering angles and the extreme-energy region $R \gtrsim b$ where a classical gravitational collapse may take place. Amati et al. proceeded a long way towards that goal from the scattering amplitude stand point in the reduced action model $[13,23]$. In such a truncated model they found that the $S$ Matrix, as functional of the UV-safe solutions, shows an impact-parameter singularity in the classical collapse region, thus causing a unitarity deficit that they were unable to circumvent by lack of information on the associated radiation (and on short distances).

We stress the point that, from the radiation point of view, we are better off with the method presented here. In fact, we have just summarized two steps: the first one yields the emission amplitudes for the single-hit process of $\delta_{0}$ exchange [and corrections thereof in $\delta(b)$, Sec. V D]; the second one performs their superposition all along the eikonal deflection, with its interference pattern. The latter may in turn feed back on higher order corrections to the scattering amplitude itself. Therefore, by applying the present method to an improved eikonal function, we could possibly provide the radiation features given those of scattering and vice versa, by thus estimating the exchange of information between them. We hope on this basis to be able to approach the classical collapse region in a smoother way, and to test in a more direct way the features to be expected from a unitary evolution of the system.

\section{ACKNOWLEDGMENTS}

We wish to thank the Galileo Galilei Institute for Theoretical Physics and the Kavli Institute for Theoretical Physics, University of California, Santa Barbara (research supported in part by the National Science Foundation under Grant No. NSF PHY1125915) where part of this work was carried out.

\section{APPENDIX A: PHYSICAL PROJECTIONS OF THE WEINBERG AND LIPATOV CURRENTS}

In this section we calculate the explicit projections of the Weinberg and Lipatov currents over physical helicity states, proving in particular Eqs. (3.4), (3.18) and (3.21), the transformation law (3.15), and the symmetry relations (3.10) and (3.48).

We work in the gauges specified in Eqs. (3.2)-(3.3) which differ from the one used in $[6,13]$ because the gauge vector $\epsilon_{L}^{\mu}$ has the subtraction $-q^{\mu} /|\boldsymbol{q}|\left(q^{\mu} /|\boldsymbol{q}|\right)$ in the forward (backward) emission case. Such subtraction is allowed by current conservation ${ }^{6}$ and is devised to suppress the longitudinal projections of external momenta in jet 2 (jet 1) which are oppositely directed. For a generic tensor current $J_{\mu \nu}$ we define

$J_{ \pm} \equiv J_{\mu \nu} \epsilon_{\mp}^{\mu \nu}=\frac{1}{2} J_{\mu \nu} \epsilon_{\mp}^{\mu} \epsilon_{\mp}^{\nu}, \quad J_{-} \equiv \frac{J}{\sqrt{2}}, \quad J_{+}=\frac{J^{*}}{\sqrt{2}}$

in terms of the basic complex vectors (note the nonstandard normalization $\epsilon_{+} \cdot \epsilon_{-}=-2$ )

$$
\begin{aligned}
\epsilon_{ \pm}^{\mu} & =\epsilon_{T}^{\mu} \pm \mathrm{i} \epsilon_{L}^{\mu} \\
& =\left(\mp \mathrm{i} \frac{|\boldsymbol{\theta}|}{1+\sqrt{1-|\boldsymbol{\theta}|^{2}}}, \mp \mathrm{i} \mathrm{e}^{\mp \mathrm{i} \phi_{\theta}}, \mathrm{e}^{\mp \mathrm{i} \phi_{\theta}}, \pm \mathrm{i} \frac{|\boldsymbol{\theta}|}{1+\sqrt{1-|\boldsymbol{\theta}|^{2}}}\right),
\end{aligned}
$$

in the forward jet, where we have $q^{3}=\omega \sqrt{1-|\boldsymbol{\theta}|^{2}}>0$, and

\footnotetext{
${ }^{6}$ The Lipatov current is exactly conserved, while the Weinberg current is conserved up to corrections of order $\mathcal{O}(\omega / E)$ which we neglect throughout the paper.
} 


$$
\begin{aligned}
\tilde{\epsilon}_{ \pm}^{\mu} & =\epsilon_{T}^{\mu} \pm \mathrm{i} \tilde{\epsilon}_{L}^{\mu} \\
& =\left( \pm \mathrm{i} \frac{|\boldsymbol{\theta}|}{1+\sqrt{1-|\boldsymbol{\theta}|^{2}}}, \pm \mathrm{ie}^{ \pm \mathrm{i} \phi_{\theta}}, \mathrm{e}^{ \pm \mathrm{i} \phi_{\theta}}, \pm \mathrm{i} \frac{|\boldsymbol{\theta}|}{1+\sqrt{1-|\boldsymbol{\theta}|^{2}}}\right)
\end{aligned}
$$

in the backward one, where we send $q^{3} \rightarrow \tilde{q}^{3}=-q^{3}<0$, leaving the other $q^{\mu}$ components unchanged. We then see by inspection that, in the small-angle kinematics, the vectors (A2) [(A3)] have negligible longitudinal projection on jet 2 (jet 1) momenta, thereby making the corresponding contributions to the currents negligible altogether, because transverse projections are anyway of order $\mathcal{O}\left(\theta_{i}^{2}\right)$ due to the lack of collinear enhancement in the opposite jet.

\section{Forward hemisphere}

We need to calculate the typical scalar products

$$
\begin{aligned}
p_{1} \cdot \epsilon_{+} & =-\mathrm{i} E\left(\theta-\Theta_{i}\right) \mathrm{e}^{-\mathrm{i} \phi_{\theta}}, \\
p_{1} \cdot q & =\frac{E \omega}{2}\left|\theta-\Theta_{i}\right|^{2}
\end{aligned}
$$

(restricting ourselves for cleanness of notation to the negative helicity projection) which are given in complex notation in terms of $\boldsymbol{\Theta}_{i} \equiv \boldsymbol{\Theta}_{1}$, that is the $\vec{p}_{1}$ incidence angle in the general parametrization (2.1). By using Eq. (A1), the $p_{1}$ contribution to the Weinberg current is then

$$
\frac{J_{W-}^{(1)}}{\kappa}=\frac{1}{2} \frac{\left(p_{1} \cdot \epsilon_{+}\right)^{2}}{p_{1} \cdot q}=-\frac{E}{\omega} \mathrm{e}^{2 \mathrm{i}\left(\phi_{\theta-\Theta_{1}}-\phi_{\theta}\right)} .
$$

Here we note the lack of collinear singularity due to the cancellation of the squared numerator with the denominator in Eq. (A4). The contribution from $p_{1}{ }^{\prime}$ is analogous, with just the replacement $\boldsymbol{\Theta}_{1} \rightarrow \boldsymbol{\Theta}_{1}^{\prime}$, while $p_{2}$ and $p_{2}{ }^{\prime}$ give negligible contributions in this gauge and hemisphere, as explained before.

Therefore, introducing the coupling $\kappa$ and adding up the relevant terms we get

$J_{W-}^{\left(\boldsymbol{\Theta}_{i}\right)}\left(q^{3}>0, \boldsymbol{\theta}, \boldsymbol{\theta}_{s}\right)=\kappa \frac{E}{\omega}\left(\mathrm{e}^{2 \mathrm{i}\left(\phi_{\theta-\Theta_{i}-\theta_{s}}-\phi_{\theta}\right)}-\mathrm{e}^{2 \mathrm{i}\left(\phi_{\theta-\boldsymbol{\theta}_{i}}-\phi_{\theta}\right)}\right)$

$$
\simeq \kappa \frac{E}{\omega} \mathrm{e}^{-2 \mathrm{i}\left(\phi_{\theta}-\phi_{\theta-\boldsymbol{\theta}_{i}}\right)}\left(\mathrm{e}^{2 \mathrm{i}\left(\phi_{\theta-\boldsymbol{\theta}_{i}-\boldsymbol{\theta}_{s}}-\phi_{\theta-\boldsymbol{\theta}_{i}}\right)}-1\right) .
$$

Here we have used the approximate relation

$$
\boldsymbol{\Theta}_{1}^{\prime}=\boldsymbol{\Theta}_{1}+\boldsymbol{\theta}_{s}+\mathcal{O}(\omega / E)
$$

neglecting the momentum conservation corrections of order $\mathcal{O}(\omega / E)$, which is allowed in regions $(\mathrm{a})+(\mathrm{b})$ where the Weinberg current is relevant and $|\boldsymbol{q}| /\left|\boldsymbol{q}_{2}\right|$ is small.
Equations (A6)-(A7) prove formulas (3.4) (in which we have $\boldsymbol{\Theta}_{i}=0$ and $\boldsymbol{\Theta}_{f}=\boldsymbol{\theta}_{s}$ ) and (3.18), thus confirming the transformation law (3.15) for the Weinberg current.

For the Lipatov current (including for convenience the denominator associated to the $q_{1}^{\mu}, q_{2}^{\mu}$ virtualities), the negative helicity projection is given by

$$
\frac{J_{L-}^{\left(\boldsymbol{\Theta}_{i}\right)}\left(q^{3}>0\right)}{\left|\boldsymbol{q}_{1 \perp}\right|^{2}\left|\boldsymbol{q}_{2 \perp}\right|^{2}}=\frac{\kappa}{4}\left(\frac{\left(J \cdot \epsilon_{+}\right)^{2}}{\left|\boldsymbol{q}_{\perp 1}\right|^{2}\left|\boldsymbol{q}_{\perp 2}\right|^{2}}-\left(j \cdot \epsilon_{+}\right)^{2}\right)
$$

[see Eq. (3.19)], where we have

$$
\begin{aligned}
\left(j \cdot \epsilon_{+}\right) & =\frac{p_{1} \cdot \epsilon_{+}}{p_{1} \cdot q}=-\frac{2 \mathrm{i}}{\omega} \frac{\mathrm{e}^{-\mathrm{i} \phi_{\theta}}}{\left(\theta-\Theta_{i}\right)^{*}}, \\
\left(J \cdot \epsilon_{+}\right) & =\left(\boldsymbol{q}_{\perp 1}^{2}-\boldsymbol{q}_{\perp}^{2}\right) \frac{-2 \mathrm{ie}^{-\mathrm{i} \phi_{\theta}}}{\omega\left(\theta-\Theta_{i}\right)^{*}}-2 q_{2} \cdot \epsilon_{+} \\
& =\left(\boldsymbol{q}_{\perp 1}^{2}-\boldsymbol{q}_{\perp}^{2}\right) \frac{-2 \mathrm{ie}-\mathrm{i} \phi_{\theta}}{\omega\left(\theta-\Theta_{i}\right)^{*}}-2 \mathrm{ie}^{-\mathrm{i} \phi_{\theta}} q_{2} .
\end{aligned}
$$

Here we have used current conservation to replace $q_{1}^{\mu}-q_{2}^{\mu} \rightarrow-2 q_{2}^{\mu}$ and $\boldsymbol{q}_{\perp 1}, \boldsymbol{q}_{\perp 2}$ and $\boldsymbol{q}_{\perp}$ denote transverse (vectorial) components with respect to the $\vec{p}_{1}$ direction (and $q_{\perp 1}, q_{\perp 2}$ and $q_{\perp}$, the corresponding complex versions), which are related to $\boldsymbol{q}_{1}, \boldsymbol{q}_{2}$ and $\boldsymbol{q}$ by a rotation of angle $\left|\boldsymbol{\Theta}_{i}\right|$ of the reference frame. Note in particular that since we are considering a forward emission, $q_{2}^{\mu}$ has practically no longitudinal component, while $q_{1}^{\mu}$ has; taking also into account that $q_{\mu}=q_{1 \mu}+q_{2 \mu}$, this implies

$$
\boldsymbol{q}_{\perp 2} \simeq \boldsymbol{q}_{2}, \quad \boldsymbol{q}_{\perp 1}-\boldsymbol{q}_{1} \simeq \boldsymbol{q}_{\perp}-\boldsymbol{q} \simeq-\omega \boldsymbol{\Theta}_{i} .
$$

Now, rewriting $q_{\perp 1}=q_{\perp}-q_{\perp 2}$, using $q_{\perp}=\omega\left(\theta-\Theta_{i}\right)$ and taking into account Eq. (A12), we can rewrite Eq. (A11) in the form

$$
\begin{aligned}
\left(J \cdot \epsilon_{+}\right) & =\frac{-2 \mathrm{ie}^{-\mathrm{i} \phi_{\theta}}}{q_{\perp}^{*}}\left(\boldsymbol{q}_{2}^{2}-2 \boldsymbol{q}_{\perp} \cdot \boldsymbol{q}_{2}+q_{\perp}^{*} q_{2}\right) \\
& =\frac{2 \mathrm{i} \mathrm{e}^{-\mathrm{i} \phi_{\theta}}}{\omega\left(\theta-\Theta_{i}\right)^{*}} q_{2}^{*} q_{\perp 1} .
\end{aligned}
$$

By substituting expression (A13) in Eq. (A9) we finally get $J_{L-}^{\left(\boldsymbol{\Theta}_{i}\right)}\left(q^{3}>0, \boldsymbol{q}, \boldsymbol{q}_{2}\right)=\frac{\kappa}{\left|\boldsymbol{q}_{\perp}\right|^{2}} \mathrm{e}^{2 \mathrm{i}\left(\phi_{\theta-\boldsymbol{\Theta}_{i}}-\phi_{\theta}\right)}\left[1-\mathrm{e}^{-2 \mathrm{i}\left(\phi_{q_{2}}-\phi_{q_{\perp}-q_{2}}\right)}\right]$,

which proves (by setting $\boldsymbol{\Theta}_{i}=0$ ) Eq. (3.21) of the text and the transformation law to general $\boldsymbol{\Theta}_{i}$ (3.15) for the Lipatov current. We note that the would-be singularities at $\boldsymbol{q}_{\perp 1}, \boldsymbol{q}_{\perp 2}=0$ have been canceled due to Eq. (A13) and replaced by the phase difference in Eq. (A14), which also reduces the $\boldsymbol{q}_{\perp}=0$ singularity to a linear integrable (in two dimensions) one. 


\section{Backward hemisphere}

In this case the jet 2 is characterized by an incidence angle $\tilde{\boldsymbol{\Theta}}_{i} \equiv \boldsymbol{\Theta}_{2}$ (which in the center-of-mass frame $\vec{p}_{1}+\vec{p}_{2}=0$ is simply provided by $\tilde{\boldsymbol{\Theta}}_{i}=-\boldsymbol{\Theta}_{1}=-\boldsymbol{\Theta}_{i}$ ) and by the fact that the emitted $q^{\mu}$ has a negative $\hat{z}$ component, $\tilde{q}^{3}=-\omega \sqrt{1-|\boldsymbol{\theta}|^{2}}<0$.

By using the backward helicity vectors (A3) we obtain

$$
\begin{gathered}
p_{2} \cdot \tilde{\epsilon}^{+}=\mathrm{i} E\left(\theta^{*}+\Theta_{i}^{*}\right) \mathrm{e}^{\mathrm{i} \phi_{\theta}}, \\
p_{2} \cdot \tilde{q}=\frac{E \omega}{2}\left|\theta+\Theta_{i}\right|^{2},
\end{gathered}
$$

and, as a consequence

$$
\frac{J_{W-}^{(2)}}{\kappa}=\frac{1}{2} \frac{\left(p_{2} \cdot \tilde{\epsilon}_{+}\right)^{2}}{p_{1} \cdot q}=-\frac{E}{\omega} \mathrm{e}^{-2 \mathrm{i}\left(\phi_{\theta-\Theta_{2}}-\phi_{\theta}\right)} .
$$

Since jet 1 is switched off, the Weinberg current is simply $J_{W-}^{\left(-\boldsymbol{\Theta}_{i}\right)}\left(-q^{3}<0, \boldsymbol{\theta}, \boldsymbol{\theta}_{s}\right)=\kappa \frac{E}{\omega} \mathrm{e}^{2 \mathrm{i} \phi_{\theta}}\left(\mathrm{e}^{-2 \mathrm{i} \phi_{\theta+\theta_{s}+\boldsymbol{\Theta}_{i}}}-\mathrm{e}^{-2 \mathrm{i} \phi_{\theta+\boldsymbol{\Theta}_{i}}}\right)$,

where we used the relation

$$
\boldsymbol{\Theta}_{2}^{\prime}=-\boldsymbol{\Theta}_{i}-\boldsymbol{\theta}_{s}+\mathcal{O}\left(\frac{\omega}{E}\right)
$$

and neglected, as in the forward case, the $\mathcal{O}(\omega / E)$ corrections in the $(a)+(b)$ regions.

We note that Eq. (A17) can be recast in a form transforming like the complex conjugate (or opposite helicity) of (A7) by the replacement $\boldsymbol{\theta} \rightarrow-\boldsymbol{\theta}$, to yield

$$
\begin{aligned}
& J_{W-}^{\left(-\boldsymbol{\Theta}_{i}\right)}\left(-q^{3}<0,-\boldsymbol{\theta}, \boldsymbol{\theta}_{S}\right) \\
& =\kappa \frac{E}{\omega} \mathrm{e}^{2 \mathrm{i}\left(\phi_{\theta}-\phi_{\theta-\Theta_{i}}\right)}\left(\mathrm{e}^{-2 \mathrm{i}\left(\phi_{\theta-\Theta_{i}-\theta_{s}}-\phi_{\theta-\Theta_{i}}\right)}-1\right) \\
& =J_{W+}^{\left(\boldsymbol{\Theta}_{i}\right)}\left(q^{3}>0, \boldsymbol{\theta}, \boldsymbol{\theta}_{s}\right) \text {. }
\end{aligned}
$$

This proves Eq. (3.9) and yields the basis for the relationship (3.10), in which we also use the F.T. with respect to $\boldsymbol{q}_{2}=E \boldsymbol{\theta}_{s}+\boldsymbol{q}$.

All that is left is to obtain the helicity projections of the Lipatov current in the backward hemisphere. Using Eqs. (3.19) and (A1) we obtain

$$
\frac{J_{L-}^{\left(-\boldsymbol{\Theta}_{i}\right)}\left(-q^{3}<0\right)}{\left|\boldsymbol{q}_{1}\right|^{2}\left|\tilde{\boldsymbol{q}}_{2 \perp}\right|^{2}}=\frac{\kappa}{4}\left(\frac{\left(J \cdot \tilde{\epsilon}_{+}\right)^{2}}{\left|\boldsymbol{q}_{1}\right|^{2}\left|\tilde{\boldsymbol{q}}_{\perp 2}\right|^{2}}-\left(j \cdot \tilde{\epsilon}_{+}\right)^{2}\right),
$$

where we used the fact that $q_{1}^{\mu}$ has negligible longitudinal components for an emission in jet 2 and defined (in complex notation)

$$
\tilde{q}_{\perp}-q \simeq \tilde{q}_{\perp 2}-q_{2} \simeq-\omega \tilde{\Theta}_{i}=\omega \Theta_{i} .
$$

The vector current projections are

$$
\begin{aligned}
\left(j \cdot \tilde{\epsilon}_{+}\right) & =\frac{p_{1} \cdot \tilde{\epsilon}_{+}}{p_{1} \cdot \tilde{q}}=-\frac{2 \mathrm{i}}{\omega} \frac{\mathrm{e}^{\mathrm{i} \phi_{\theta}}}{\left(\theta+\Theta_{i}\right)}, \\
\left(J \cdot \tilde{\epsilon}_{+}\right) & =\left(\tilde{\boldsymbol{q}}_{\perp 2}^{2}-\tilde{\boldsymbol{q}}_{\perp}^{2}\right)\left(j \cdot \tilde{\epsilon}_{+}\right)+2 q_{1} \cdot \tilde{\epsilon}_{+} \\
& =\frac{2 \mathrm{e}^{\mathrm{i} \phi_{\theta}}}{\tilde{\boldsymbol{q}}_{\perp}} \tilde{q}_{\perp 2}^{*} q_{1},
\end{aligned}
$$

where we performed the algebra along the lines explained before. We thus obtain

$$
\begin{aligned}
& J_{L-}^{\left(-\boldsymbol{\Theta}_{i}\right)}\left(-q^{3}<0, \boldsymbol{q}, \boldsymbol{q}_{1}\right) \\
& \quad=\frac{\kappa}{\left|\tilde{\boldsymbol{q}}_{\perp}\right|^{2}} \mathrm{e}^{-2 \mathrm{i}\left(\phi_{\boldsymbol{\theta}+\boldsymbol{\Theta}_{i}}-\phi_{\theta}\right)}\left[1-\mathrm{e}^{2 \mathrm{i}\left(\phi_{q_{1}}-\phi_{\tilde{q}_{\perp}-q_{1}}\right)}\right] .
\end{aligned}
$$

At $\boldsymbol{\Theta}_{i}=0$ the above result agrees with that in the forward jet by the trivial replacement $\boldsymbol{q}_{1}=\boldsymbol{q}-\boldsymbol{q}_{2}$, meaning that the Regge limit yields the same form of the amplitude in either jet. On the other hand, at nonzero $\boldsymbol{\Theta}_{i}$, and by the replacement $\boldsymbol{q} \rightarrow-\boldsymbol{q}, \boldsymbol{q}_{1} \rightarrow-\boldsymbol{q}_{2}$, it yields the helicity relation

$J_{L-}^{\left(-\boldsymbol{\Theta}_{i}\right)}\left(-q^{3}<0,-\boldsymbol{q},-\boldsymbol{q}_{2}\right)=J_{L+}^{\left(\boldsymbol{\Theta}_{i}\right)}\left(q^{3}>0, \boldsymbol{q}, \boldsymbol{q}_{2}\right) ;$

by finally using the transformation $\tilde{\boldsymbol{q}}_{\perp 2} \rightarrow-\boldsymbol{q}_{\perp}+\boldsymbol{q}_{2}$ in the F.T. under the same replacement, we prove Eq. (3.48) of the text.

\section{3. $z$ representation}

A generic phase difference of the form $\mathrm{e}^{2 \mathrm{i} \phi_{\theta}}-\mathrm{e}^{2 \mathrm{i} \phi_{\theta^{\prime}}}$, with $\boldsymbol{\theta}, \boldsymbol{\theta}^{\prime}$ generic 2-vectors, of the kind that appears in the physical projections of both the Weinberg (3.8) and the Regge (3.22) amplitudes, can be conveniently rewritten in integral form:

$$
\mathrm{e}^{2 \mathrm{i} \phi_{\theta}}-\mathrm{e}^{2 \mathrm{i} \phi_{\theta^{\prime}}}=-2 \int \frac{\mathrm{d}^{2} z}{2 \pi z^{* 2}}\left(\mathrm{e}^{\mathrm{i} A z \cdot \boldsymbol{\theta}}-\mathrm{e}^{\mathrm{i} A z \cdot \boldsymbol{\theta}^{\prime}}\right),
$$

where $A$ is an arbitrary scale (the integration measure $\mathrm{d}^{2} z / z^{* 2}$ in the integral is scale invariant). This is easily verified by performing first the azimuthal, then the radial integration in $\mathrm{d}^{2} z$. We get (setting for simplicity $A=1$ )

$$
\begin{aligned}
\int & \frac{\mathrm{d}^{2} z}{2 \pi z^{* 2}}\left(\mathrm{e}^{\mathrm{i} z \cdot \boldsymbol{\theta}}-\mathrm{e}^{\mathrm{i} z \cdot \boldsymbol{\theta}^{\prime}}\right) \\
= & -\int_{0}^{\infty} \frac{\mathrm{d}|z|}{|z|}\left(\mathrm{e}^{2 \mathrm{i} \phi_{\theta}} J_{2}(|z \| \boldsymbol{\theta}|)-\mathrm{e}^{2 \mathrm{i} \phi_{\theta}} J_{2}\left(\left|z \| \boldsymbol{\theta}^{\prime}\right|\right)\right) \\
& =-\frac{1}{2}\left(\mathrm{e}^{2 \mathrm{i} \phi_{\boldsymbol{\theta}}}-\mathrm{e}^{2 \mathrm{i} \phi_{\theta^{\prime}}}\right),
\end{aligned}
$$

where in the last step we used the scale invariance of the integration measure, the standard integration formula $\int \frac{J_{2}(x)}{x}=-\frac{J_{1}(x)}{x}$ and the fact that $J_{1}(x) \sim x / 2$ near $x=0$. Equation (A25) is thus proven. 


\section{APPENDIX B: TRANSFORMATION OF HELICITY AMPLITUDES}

In this section we derive the transformation formula for amplitudes of definite helicity when the momentum of the incoming particle undergoes a rotation. We are mainly interested in small emission and deflection angles. Therefore in Sec. B 1 we derive some simple properties of small rotations that will then be applied in Sec. B 2 in order to obtain the transformation formulas.

\section{Small-angle rotations}

In the eikonal approximation, the typical polar angles of the particles are small. This means that the 3 -momentum of a particle in the forward region can be written as

$$
\vec{q}=\left(\boldsymbol{q}, q_{z}\right) \equiv \omega\left(\boldsymbol{\theta}, \sqrt{1-\boldsymbol{\theta}^{2}}\right) \simeq \omega(\boldsymbol{\theta}, 1),
$$

where $\omega=|\vec{q}|$ and $\boldsymbol{\theta} \equiv \boldsymbol{q} /|\vec{q}|$ is the intersection of $\vec{q}$ with the tangent plane to the unit sphere at $\hat{z}=(0,0,1)$, so that $|\boldsymbol{\theta}|$ can be interpreted as polar angle when $|\boldsymbol{\theta}| \ll 1$. Note that such a parametrization spans only half of the phase space. The backward hemisphere is described by an analogous transverse vector spanning the tangent plane at $-\hat{z}=(0,0,-1)$.

Any unit-vector $\hat{q}$ can be obtained by applying to $\hat{z}$ a rotation around an axis on the $\langle x, y\rangle$ plane:

$$
\hat{q}=R(\vec{\alpha}) \hat{z}=\exp \left\{-\mathrm{i}\left(L_{x} \alpha_{x}+L_{y} \alpha_{y}\right)\right\} \hat{z},
$$

where the matrices $\left(L_{k}\right)_{m n}=-\mathrm{i} \varepsilon_{k m n}: k=1,2,3$ are the generators of rotations and $\vec{\alpha}=\left(\alpha_{x}, \alpha_{y}, 0\right)$ denotes the rotation vector. For small rotation angles $|\vec{\alpha}| \ll 1$, the exponentials can be expanded to first order and one finds

$$
\begin{aligned}
&\left(\theta_{x}, \theta_{y}, 1\right)= {\left[1-\mathrm{i}\left(L_{x} \alpha_{x}+L_{y} \alpha_{y}\right)\right] \hat{z}+\mathcal{O}\left(\vec{\alpha}^{2}\right) } \\
& \text { with }\left\{\begin{array}{l}
\alpha_{x}=\theta_{y} \\
\alpha_{y}=-\theta_{x} .
\end{array}\right.
\end{aligned}
$$

In practice, small-angle rotations act as Abelian translations on the transverse components of unit vectors:

$$
\begin{array}{r}
R\left(\vec{\alpha}_{1}\right) R\left(\vec{\alpha}_{2}\right) \hat{z} \simeq R\left(\vec{\alpha}_{1}\right)\left(\boldsymbol{\theta}_{2}, 1\right) \simeq\left(\boldsymbol{\theta}_{1}+\boldsymbol{\theta}_{2}, 1\right) \simeq R\left(\vec{\alpha}_{1}+\vec{\alpha}_{2}\right) \hat{z}, \\
\left(\left|\vec{\alpha}_{j}\right| \ll 1\right)
\end{array}
$$

up to quadratic terms in $\vec{\alpha}_{1,2}$.

\section{Amplitude transformation}

We are looking for a relation that connects a generic helicity amplitude with arbitrary incoming momentum $\vec{p}_{i}$ $\left(\boldsymbol{\Theta}_{i} \neq 0\right)$ to another amplitude having incoming momentum along the $\hat{z}$ axis $\left(\boldsymbol{\Theta}_{i}=0\right)$. Our procedure is based on two main observations:
(1) helicity amplitudes are invariant under rigid rotations of all momenta and polarization vectors; by applying a suitable rotation we can bring the incoming momentum $\vec{p}_{i}$ onto the $\hat{z}$ axis;

(2) the rotated polarization vectors differ from the reference ones [Eqs. (3.3)] by a further rotation around the emitted graviton momentum; this is the rotation providing the helicity phase factor of the amplitude transformation.

Let us discuss the two points in more detail.

(1) A helicity amplitude $A^{(\lambda)}$ is defined by contracting a tensor amplitude $A^{\mu \nu}$ with some polarization tensor $\epsilon_{\mu \nu}^{(\lambda)}$, e.g., $A^{(\lambda)}\left(p, q, \epsilon^{*}\right)=A^{\mu \nu}(p, q) \epsilon_{\mu \nu}^{(\lambda) *}$. The notation shows that $A^{(\lambda)}$ explicitly depends on the emitted momentum $q$, the polarization vector $\epsilon^{*}$ and additional momenta of the process denoted by $p$. Of course, $A^{(\lambda)}$ is invariant under Lorentz transformations of $p, q$ and $\epsilon$, and, in particular, under any spatial rotation $R: A^{(\lambda)}\left(R p, R q, R \epsilon^{*}\right)=A^{(\lambda)}\left(p, q, \epsilon^{*}\right)$.

In order to specify the polarization tensor $\epsilon^{(\lambda)}$ (and the helicity amplitude), one has to uniquely define a pair of polarization vectors $\epsilon_{k}: k=1,2$ which are orthogonal to the momentum $q$ of the emitted radiation. Usually such vectors are chosen without time components, of unit length and orthogonal to each other, in such a way that $\left\{\vec{q}, \vec{\epsilon}_{1}, \vec{\epsilon}_{2}\right\}$ forms a right-hand basis of 3-space. The remaining degree of freedom is a rotation of the pair $\left\{\vec{\epsilon}_{1}, \vec{\epsilon}_{2}\right\}$ around the $\vec{q}$ axis. In this paper, $\epsilon_{1}=\epsilon_{T}$ and $\epsilon_{2}=\epsilon_{L}+$ gauge-term $\propto q$.

We completely specify the polarizations by requiring $\vec{\epsilon}_{T}$ to be orthogonal to a given momentum $\vec{p}$ : $\vec{\epsilon}_{T} \cdot \vec{p}=0$. According to this recipe, we can write (omitting the three-dimensional arrows) $\epsilon_{k}=$ $\epsilon_{k}(p, q)$. Such a procedure is frame independent; therefore we have

$$
\epsilon_{k}(R p, R q)=R \epsilon_{k}(p, q),
$$

meaning that if we simultaneously rotate $p$ and $q$, the polarization vectors $\epsilon_{k}$ necessarily get rotated by the same matrix $R$. The same holds for $\epsilon_{k}^{*}$.

In this paper we chose $p_{1}=E \hat{z}$ as reference vector orthogonal to $\epsilon_{T}$, and defined accordingly

$$
M^{\left(\boldsymbol{\Theta}_{i}\right)}\left(\boldsymbol{\Theta}_{f}, \boldsymbol{\theta}\right) \equiv A^{(\lambda)}\left(p_{i}, p_{f}, q, \epsilon^{*}\left(p_{1}, q\right)\right),
$$

$p_{i}$ and $p_{f}$ being the incident and final momenta of the fast particle. We can relate this amplitude to a zero-incidence-angle one by applying a rotation $R$ to the arguments of $A^{(\lambda)}$, in such a way that $p_{i}^{\prime} \equiv R p_{i}=p_{1}$ :

$$
\begin{aligned}
M^{\left(\boldsymbol{\Theta}_{i}\right)}\left(\boldsymbol{\Theta}_{f}, \boldsymbol{\theta}\right) & =A^{(\lambda)}\left(R p_{i}, R p_{f}, R q, R \epsilon^{*}\left(p_{1}, q\right)\right) \\
& =A^{(\lambda)}\left(p_{1}, R p_{f}, R q, \epsilon^{*}\left(R p_{1}, R q\right)\right) \\
& \equiv A^{(\lambda)}\left(p_{1}, p_{f}^{\prime}, q^{\prime}, \epsilon^{*}\left(p_{1}^{\prime}, q^{\prime}\right)\right)
\end{aligned}
$$


where a prime means "rotated by $R$ " and we have used Eq. (B5) in the second step.

In the case of small polar angles $\left(\boldsymbol{\theta}, \boldsymbol{\Theta}_{i}, \boldsymbol{\Theta}_{f} \ll 1\right)$ we can write $q=\omega(\boldsymbol{\theta}, 1), p_{i}=E\left(\boldsymbol{\Theta}_{i}, 1\right)$ etc., and the previous rotation $R$ amounts just to a translation of $-\boldsymbol{\Theta}_{i}$ on the angular variables: $q^{\prime}=\omega\left(\boldsymbol{\theta}-\boldsymbol{\Theta}_{i}, 1\right)$, $p_{1}^{\prime}=E\left(-\boldsymbol{\Theta}_{i}, 1\right)$ and so on.

(2) The last quantity in Eq. (B7) would be just $M^{(\boldsymbol{(})}\left(\boldsymbol{\Theta}_{f}-\boldsymbol{\Theta}_{i}, \boldsymbol{\theta}-\boldsymbol{\Theta}_{i}\right)$, if only $p_{1}$ (and not $p_{1}^{\prime}$ ) would appear "inside" the polarization $\epsilon^{*}$. But $\epsilon\left(p_{1}^{\prime}, q^{\prime}\right)$, being orthogonal to $q^{\prime}$, is obtained by applying to $\epsilon\left(p_{1}, q^{\prime}\right)$ a rotation around $q^{\prime}$ :

$\epsilon^{*}\left(p_{1}^{\prime}, q^{\prime}\right)=R_{q^{\prime}}(\alpha) \epsilon^{*}\left(p_{1}, q^{\prime}\right)=\mathrm{e}^{\mathrm{i} \lambda \alpha} \epsilon^{*}\left(p_{1}, q^{\prime}\right)$

where $\alpha$ is a suitable (possibly large) rotation angle, and we have exploited the fact that a polarization tensor of helicity $\lambda$ acquires a phase factor under rotations. To sum up,

$$
M^{\left(\boldsymbol{\Theta}_{i}\right)}\left(\boldsymbol{\Theta}_{f}, \boldsymbol{\theta}\right)=\mathrm{e}^{\mathrm{i} \lambda \alpha} M^{(\boldsymbol{\theta})}\left(\boldsymbol{\Theta}_{f}-\boldsymbol{\Theta}_{i}, \boldsymbol{\theta}-\boldsymbol{\Theta}_{i}\right) .
$$

It remains to compute the rotation angle $\alpha$. This is easily derived by rotating the original system by the (small) angle $\boldsymbol{- \theta}$ in such a way that $q \rightsquigarrow q^{\prime \prime}$ is put on the $z$ axis so that the $\left\langle q^{\prime \prime}\right\rangle^{\perp}$ plane becomes the transverse plane, as depicted in Fig. 14. Explicitly,

$$
\begin{aligned}
& q=\omega(\boldsymbol{\theta}, 1) \quad \rightsquigarrow q^{\prime \prime}=\omega(\mathbf{0}, 1) \\
& p_{i}=E\left(\boldsymbol{\Theta}_{i}, 1\right) \rightsquigarrow p_{i}^{\prime \prime}=E\left(\boldsymbol{\Theta}_{i}-\boldsymbol{\theta}, 1\right) \\
& p_{1}=E(-\mathbf{0}, 1) \rightsquigarrow p_{1}^{\prime \prime}=E(-\boldsymbol{\theta}, 1)
\end{aligned}
$$

It is now evident that the azimuth of $p_{1}^{\prime \prime}$ in the $\left\langle q^{\prime \prime}\right\rangle^{\perp}$ plane is $\varphi_{p_{1}^{\prime \prime}}=\phi_{-\theta}=\phi_{\theta}+\pi$ and that of $p_{i}^{\prime \prime}$ is $\varphi_{p_{i}^{\prime \prime}}=\phi_{\boldsymbol{\Theta}_{i}-\boldsymbol{\theta}}=\phi_{\boldsymbol{\theta}-\boldsymbol{\Theta}_{i}}+\pi$, and their difference is just the sought rotation angle

$$
\alpha=\varphi_{p_{1}^{\prime \prime}}-\varphi_{p_{i}^{\prime \prime}}=\phi_{\boldsymbol{\theta}}-\phi_{\boldsymbol{\theta}-\boldsymbol{\Theta}_{i}},
$$

because the $\epsilon_{T} \mathrm{~s}$ are orthogonal to the projections of $p_{i}$ and $p_{1}$ on the $\langle q\rangle^{\perp}$ plane.

The same reasoning applies in the case of backward emission, e.g., with $\vec{q}=\omega(\tilde{\boldsymbol{\theta}},-1)$ directed in the opposite direction with respect to Fig. 14. In this case the rotation angle needed to align $q$ along $-\hat{z}$ is just $\boldsymbol{\theta}=-\tilde{\boldsymbol{\theta}}$. Furthermore, the azimuthal angle $\alpha$ between the polarization vectors must be counted in the opposite direction, because the thumb of the right hand now points towards the negative $z$ direction. In conclusion

$$
\tilde{\alpha}=-\left(\phi_{-\tilde{\boldsymbol{\theta}}}-\phi_{-\tilde{\boldsymbol{\theta}}-\boldsymbol{\Theta}_{i}}\right)=-\left(\phi_{\tilde{\boldsymbol{\theta}}}-\phi_{\tilde{\boldsymbol{\theta}}+\boldsymbol{\Theta}_{i}}\right) .
$$

\section{Relation with Jacob-Wick conventions}

The relation of our helicity amplitudes with those defined by JW [39] can be understood by comparing in the two frameworks the choice of the polarization vectors for a generic graviton 3-momentum $q$ with polar angle $\theta$ and azimuth $\phi$.

Let $q=R(\omega \hat{z})$, where, according to JW conventions, $R$ is the rotation matrix of angle $\theta$ and axis along $\hat{z} \times q$ (thus belonging to the transverse plane). Such a matrix $R$ is conveniently written in terms of the usual Euler angles $(\alpha, \beta, \gamma)=(\phi, \theta,-\phi)$, so that it can be represented as the product of three rotations along the $y$ and $z$ axis:

$$
R=R_{\phi, \theta,-\phi}=R_{z}(\phi) R_{y}(\theta) R_{z}(-\phi) .
$$

JW define a reference helicity state when the particle (here the graviton) has momentum $\omega \hat{z}$ along the positive $z$ axis. This means that they implicitly fix a pair of polarization vectors orthogonal to $\omega \hat{z}$, i.e., $\epsilon_{1}=\hat{y}$ and $\epsilon_{2}=-\hat{x}$, so as to build the right-hand orthogonal basis $\left\{\omega \hat{z}, \epsilon_{1}, \epsilon_{2}\right\}$. The transformation of the helicity state in Eq. (6) of [39]
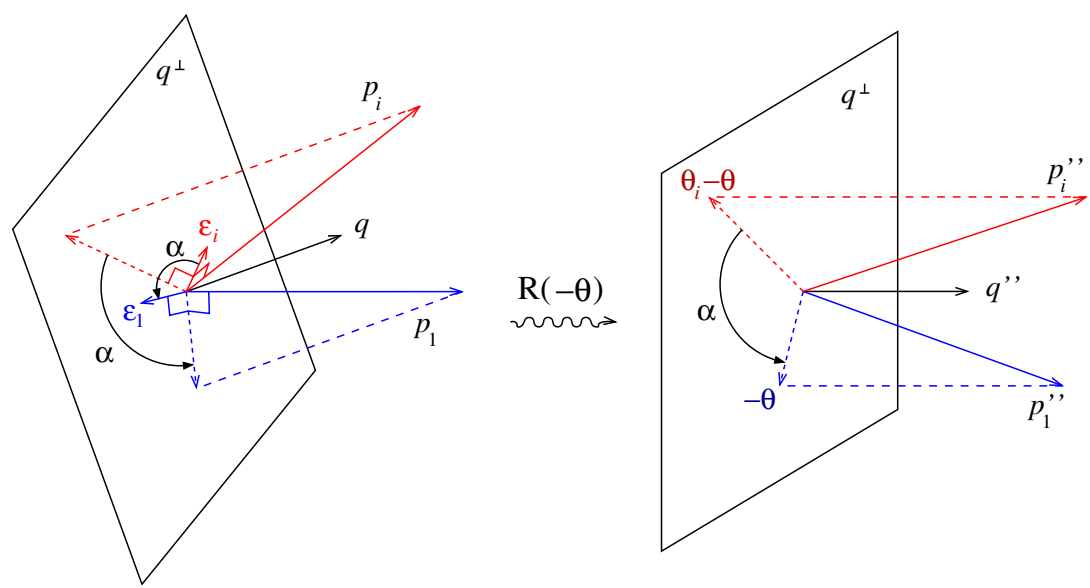

FIG. 14. Spatial representation of the graviton momentum $q$ (black) and the two \{reference-momentum, $T$-polarization $\}$ pairs $\left\{p_{1}, \epsilon_{T}\left(q, p_{1}\right)\right\}$ (blue) and $\left\{p_{i}, \epsilon_{T}\left(q, p_{i}\right)\right\}$ (red) in the original frame (left) and in the rotated frame (right) where $q \rightsquigarrow q^{\prime \prime}$ lies on the $z$ axis. 
corresponds to rotate the graviton momentum and the polarization vectors with the matrix $R$ of Eq. (B12), in such a way that the right-hand basis adapted to $q$ is $\left\{q, R \epsilon_{1}, R \epsilon_{2}\right\}$.

On the other hand, our convention (3.3) of the polarization vectors requires $\epsilon_{T}$ to be orthogonal to both $q$ and $\hat{z}$, and it is easy to see that

$$
\epsilon_{T}=R_{\phi, \theta, 0} \epsilon_{1}=R_{z}(\phi) R_{y}(\theta) \epsilon_{1},
$$

where the rotation matrix $R_{\phi, \theta, 0}$ differs from that of JW by the vanishing of the last Euler angle $\phi$, which does not affect the action $\omega \hat{z} \rightarrow q$, but changes the orientation of the polarization vectors in the $\langle q\rangle^{\perp}$ plane.

In practice, our right-hand basis $\left\{q, \epsilon_{T}, \epsilon_{L}\right\}$ is obtained by applying to the JW reference basis $\left\{\omega \hat{z}, \epsilon_{1}, \epsilon_{2}\right\}$ the rotation $R_{\phi, \theta, 0}$ of Eq. (B13). As a consequence, our polarization vectors are rotated by an angle $+\phi$ around $q$ with respect to those of JW. It follows that $2^{-1 / 2} \epsilon_{ \pm}=$ $R_{q}(\phi) \epsilon_{ \pm}^{\mathrm{JW}}=\mathrm{e}^{\mp \mathrm{i} \phi} \epsilon_{ \pm}^{\mathrm{JW}}$ while for the helicity amplitudes (involving contractions with $\epsilon_{ \pm}^{\mu \nu *}$ ) we have

$$
M_{\lambda}(q)=\mathrm{e}^{\mathrm{i} \lambda \phi} M_{\lambda}^{\mathrm{JW}}(q) .
$$

Let us now rederive the amplitude transformation phase of Eqs. (B9)-(B10). In the JW conventions, the amplitudes are invariant under rotations bringing $\omega \hat{z} \leftrightarrow q$, provided the rotation axis is in the transverse plane. In the case of small emission angles $\boldsymbol{\theta} \ll 1$, adopting now the two-dimensional angular notations, such rotations are translations in the transverse components of forward momenta like $q, p_{i}, p_{f}$, as explained in the previous subsections B 1-B 2 . Therefore, starting with the amplitude $M_{\lambda}^{\mathrm{JW}\left(\boldsymbol{\Theta}_{i}\right)}\left(\boldsymbol{\Theta}_{f}, \boldsymbol{\theta}\right)$ and applying first a small (JW-like) rotation $R(-\boldsymbol{\theta})$ bringing $q \rightarrow \omega \hat{z}$ and then another small (JW-like) rotation $R\left(\boldsymbol{\Theta}_{i}-\boldsymbol{\theta}\right)$ we find that

$$
\begin{aligned}
M_{\lambda}^{\mathrm{JW}\left(\boldsymbol{\Theta}_{i}\right)}\left(\boldsymbol{\Theta}_{f}, \boldsymbol{\theta}\right) & =M_{\lambda}^{\mathrm{JW}\left(\boldsymbol{\Theta}_{i}-\boldsymbol{\theta}\right)}\left(\boldsymbol{\Theta}_{f}-\boldsymbol{\theta}, \boldsymbol{0}\right) \\
& =M_{\lambda}^{\mathrm{JW}(\mathbf{0})}\left(\boldsymbol{\Theta}_{f}-\boldsymbol{\Theta}_{i}, \boldsymbol{\theta}-\boldsymbol{\Theta}_{i}\right),
\end{aligned}
$$

up to terms $\mathcal{O}\left(\boldsymbol{\theta}, \boldsymbol{\Theta}_{i}\right)^{2}$ in the arguments of $M^{\mathrm{JW}}$. By then recalling Eq. (B14) we immediately obtain

$$
\begin{aligned}
M_{\lambda}^{\left(\boldsymbol{\Theta}_{i}\right)}\left(\boldsymbol{\Theta}_{f}, \boldsymbol{\theta}\right) & \stackrel{(\mathrm{B} .14)}{=} \mathrm{e}^{\mathrm{i} \lambda \phi_{\boldsymbol{\theta}}} M_{\lambda}^{\mathrm{JW}\left(\boldsymbol{\Theta}_{i}\right)}\left(\boldsymbol{\Theta}_{f}, \boldsymbol{\theta}\right) \\
& \stackrel{(\mathrm{B} .15)}{=} \mathrm{e}^{\mathrm{i} \lambda \phi_{\boldsymbol{\theta}}} M_{\lambda}^{\mathrm{JW}(\mathbf{0})}\left(\boldsymbol{\Theta}_{f}-\boldsymbol{\Theta}_{i}, \boldsymbol{\theta}-\boldsymbol{\Theta}_{i}\right) \\
& \stackrel{(\mathrm{B} .14)}{=} \mathrm{e}^{\mathrm{i} \lambda \phi_{\theta}} \mathrm{e}^{-\mathrm{i} \lambda \phi_{\theta-\boldsymbol{\Theta}_{i}}} M_{\lambda}^{(\mathbf{0})}\left(\boldsymbol{\Theta}_{f}-\boldsymbol{\Theta}_{i}, \boldsymbol{\theta}-\boldsymbol{\Theta}_{i}\right) .
\end{aligned}
$$

\section{APPENDIX C: THE $h$ FIELD}

\section{Calculation of the $\boldsymbol{h}$ field in coordinate space}

According to the analysis of [13], the two real components $h_{T T}$ and $h_{L T}$ of the radiation field are conveniently collected into a single complex-valued field $h \equiv h_{T T}+\mathrm{i} h_{L T}$ that admits the integral representation [see Eq. (2.14) of [13]]

$h(\boldsymbol{b}, \boldsymbol{x})=2 \int \frac{\mathrm{d}^{2} \boldsymbol{q}_{1}}{(2 \pi)^{2}} \frac{\mathrm{d}^{2} \boldsymbol{q}_{2}}{(2 \pi)^{2}} \frac{1-\mathrm{e}^{2 \mathrm{i} \phi_{12}}}{\left(\boldsymbol{q}_{1}+\boldsymbol{q}_{2}\right)^{2}} \mathrm{e}^{-\mathrm{i}\left[\boldsymbol{q}_{1} \cdot \boldsymbol{x}+\boldsymbol{q}_{2} \cdot(\boldsymbol{x}-\boldsymbol{b})\right]}$,

where $\phi_{i j} \equiv \phi_{i}-\phi_{j}$.

By denoting with $A \equiv|\boldsymbol{x}|$ and $B \equiv|\boldsymbol{x}-\boldsymbol{b}|$ the moduli of the external vectors (see Fig. 15) appearing in the last exponent, and by explicitly writing out the various azimuthal angles, we rewrite $h$ in the form

$$
\begin{aligned}
h= & \frac{1}{2 \pi^{2}} \int_{0}^{\infty} \mathrm{d} q_{1} q_{1} \int_{0}^{\infty} \mathrm{d} q_{2} q_{2} \int_{0}^{2 \pi} \frac{\mathrm{d} \phi_{1}}{2 \pi} \\
& \times \int_{0}^{2 \pi} \frac{\mathrm{d} \phi_{2}}{2 \pi} \frac{\left(1-\mathrm{e}^{2 \mathrm{i} \phi_{12}}\right) \mathrm{e}^{-\mathrm{i}\left(q_{1} A \cos \phi_{A 1}+q_{2} B \cos \phi_{2 B}\right)}}{\left(q_{1}+q_{2} \mathrm{e}^{\mathrm{i} \phi_{12}}\right)\left(q_{1}+q_{2} \mathrm{e}^{-\mathrm{i} \phi_{12}}\right)},
\end{aligned}
$$

where $q_{i} \equiv\left|\boldsymbol{q}_{i}\right|$ and $\phi_{A}\left(\phi_{B}\right)$ is the azimuthal angle of the two-dimensional vector $\boldsymbol{x}(\boldsymbol{x}-\boldsymbol{b})$. Since $\phi_{A B}=$ $\phi_{A 1}+\phi_{12}+\phi_{2 B}$, the integrations over $\phi_{1}$ and $\phi_{2}$ actually provide a double convolution, which can be diagonalized by a Fourier transform. In practice, by computing the partial waves with respect to the angle $\phi_{A B}$, we obtain

$$
\begin{aligned}
h_{m}(A, B) \equiv & \int_{0}^{2 \pi} \frac{\mathrm{d} \phi_{A B}}{2 \pi} \mathrm{e}^{\mathrm{i} m \phi_{A B}} h(\boldsymbol{b}, \boldsymbol{x}) \\
= & \frac{(-1)^{m}}{2 \pi^{2}} \int \mathrm{d} q_{1} q_{1} J_{m}\left(q_{1} A\right) \int \mathrm{d} q_{2} q_{2} J_{m}\left(q_{2} B\right) \\
& \times \int \frac{\mathrm{d} \phi_{12}}{2 \pi} \frac{\mathrm{e}^{\mathrm{i} m \phi_{12}}\left(1-\mathrm{e}^{2 \mathrm{i} \phi_{12}}\right)}{\left(q_{1}+q_{2} \mathrm{e}^{\mathrm{i} \phi_{12}}\right)\left(q_{1}+q_{2} \mathrm{e}^{-\mathrm{i} \phi_{12}}\right)},
\end{aligned}
$$

where we used the relation

$$
\int_{0}^{2 \pi} \frac{\mathrm{d} \phi}{2 \pi} \mathrm{e}^{\mathrm{i} m \phi} \mathrm{e}^{-\mathrm{i} x \cos \phi}=\mathrm{i}^{-m} J_{m}(x) \quad(m \in \mathbb{Z}) .
$$

The last (azimuthal) integral in Eq. (C3) is easily computed by transforming it in a contour integral over the unitary circle in the complex plane of the variable $z \equiv \mathrm{e}^{\mathrm{i} \phi_{12}}$. For $m \geq 0$ we have

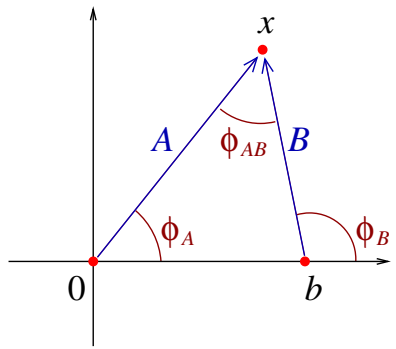

FIG. 15. The variables of the transverse plane introduced in the computation of $h(\boldsymbol{x})$. Here the impact parameter $\boldsymbol{b}$ defines the origin of azimuthal angles, i.e., the real axis of the corresponding complex plane. 


$$
\begin{aligned}
\Phi_{m}\left(q_{1}, q_{2}\right) & \equiv \int \frac{\mathrm{d} z}{2 \pi \mathrm{i}} \frac{z^{m}\left(1-z^{2}\right)}{\left(q_{1}+q_{2} z\right)\left(q_{1} z+q_{2}\right)} \\
& =(-1)^{m}\left(\frac{q_{<}}{q_{>}}\right)^{m} \frac{1}{q_{>}^{2}} \quad(m \geq 0)
\end{aligned}
$$

since only the pole at $z=-q_{<} / q_{>}$is enclosed by the contour.

For $m=-1$ the additional pole at $z=0$ provides a contribution that exactly cancels the one at $z=-q_{<} / q_{>}$: $\Phi_{-1}\left(q_{1}, q_{2}\right)=0$.

The azimuthal integral for $m \leq-2$, after the change of variable $z \rightarrow 1 / z$, keeps its original structure, determining the (anti)symmetry property $\Phi_{m}=-\Phi_{-m-2}$. Note also that $\Phi_{m}$ is symmetric in the exchange $q_{1} \leftrightarrow q_{2}$.

Let us then proceed with the computation of $h_{m}(A, B)$ for $m \geq 0$. We have

$$
\begin{aligned}
2 \pi^{2} h_{m}(A, B)= & \int_{0}^{\infty} \mathrm{d} q_{1} \mathrm{~d} q_{2} J_{m}\left(q_{1} A\right) J_{m}\left(q_{2} B\right)\left(\frac{q_{<}}{q_{>}}\right)^{m+1} \\
= & \int_{0}^{\infty} \mathrm{d} q_{1} \int_{0}^{q_{1}} \mathrm{~d} q_{2} J_{m}\left(q_{1} A\right) J_{m}\left(q_{2} B\right)\left(\frac{q_{2}}{q_{1}}\right)^{m+1} \\
& +\{A \leftrightarrow B\} .
\end{aligned}
$$

By expressing the $q_{2}$ variable in terms of the ratio $\rho \equiv q_{2} / q_{1}$, the $q_{1}$ integration reduces to the orthogonality relation for Bessel functions:

$$
\begin{aligned}
2 \pi^{2} h_{m}(A, B)= & \int_{0}^{1} \mathrm{~d} \rho \rho^{m+1} \int_{0}^{\infty} \mathrm{d} q_{1} q_{1} J_{m}\left(q_{1} A\right) J_{m}\left(q_{1} \rho B\right) \\
& +\{A \leftrightarrow B\} \\
= & \frac{1}{A B}\left(\frac{A}{B}\right)^{m+1} \Theta(B-A)+\{A \leftrightarrow B\} . \quad(\mathrm{C} 7)
\end{aligned}
$$

The $h$ field can now be obtained by summing the Fourier series

$$
\begin{aligned}
2 \pi^{2} h(\boldsymbol{x})= & \sum_{m=-\infty}^{\infty} h_{m}(A, B) \mathrm{e}^{-\mathrm{i} m \phi_{A B}} \\
= & \sum_{m=0}^{\infty}\left[h_{m}(A, B)-h_{-m-2}(A, B)\right] \mathrm{e}^{-\mathrm{i} m \phi_{A B}} \\
= & \sum_{m=0}^{\infty} \frac{\mathrm{e}^{-\mathrm{i} m \phi_{A B}}-\mathrm{e}^{\mathrm{i}(m+2) \phi_{A B}}}{A B} \\
& \times\left[\left(\frac{A}{B}\right)^{m+1} \Theta(B-A)+\left(\frac{B}{A}\right)^{m+1} \Theta(A-B)\right] \\
= & \frac{1}{\boldsymbol{A}^{*} \boldsymbol{B}}\left[\Theta(B-A)\left(\frac{\boldsymbol{A}^{*}}{\boldsymbol{B}^{*}-\boldsymbol{A}^{*}}-\frac{\boldsymbol{A}}{\boldsymbol{B}-\boldsymbol{A}}\right)\right. \\
& \left.+\Theta(A-B)\left(\frac{\boldsymbol{B}_{\boldsymbol{A}}-\boldsymbol{B}}{\boldsymbol{A}^{*}-\boldsymbol{B}^{*}}\right)\right]
\end{aligned}
$$

where we introduced the complex numbers $\boldsymbol{A} \equiv A \mathrm{e}^{\mathrm{i} \phi_{A}}$ and $\boldsymbol{B} \equiv B \mathrm{e}^{\mathrm{i} \phi_{B}}$. It turns out that the square brackets in the last equation are equal, and we finally obtain

$h(\boldsymbol{x})=\frac{1}{2 \pi^{2}} \frac{\boldsymbol{A}^{*} \boldsymbol{B}-\boldsymbol{A} \boldsymbol{B}^{*}}{\boldsymbol{A}^{*} \boldsymbol{B}|\boldsymbol{A}-\boldsymbol{B}|^{2}}=\frac{1}{2 \pi^{2}} \frac{x-x^{*}}{b x^{*}(x-b)}=\frac{1-\mathrm{e}^{2 \mathrm{i} \phi_{A B}}}{2 \pi^{2} b^{2}}$.

The components $h_{T T}$ and $h_{L T}$ correspond to the real and imaginary part of $h$, respectively, and read $\left(\phi_{A}=\phi_{x b}\right)$

$h_{T T}(\boldsymbol{x} ; \boldsymbol{b})=\frac{1-\cos \left(2 \phi_{A B}\right)}{2 \pi^{2} b^{2}}=\frac{\sin ^{2} \phi_{A B}}{\pi^{2} b^{2}}=\frac{\sin ^{2} \phi_{x b}}{\pi^{2}|\boldsymbol{x}-\boldsymbol{b}|^{2}}$

$h_{L T}(\boldsymbol{x} ; \boldsymbol{b})=\frac{-\sin \left(2 \phi_{A B}\right)}{2 \pi^{2} b^{2}}=\frac{\sin \phi_{x b}}{\pi^{2}|\boldsymbol{x}-\boldsymbol{b}|^{2}}\left(\frac{|\boldsymbol{x}|}{b}-\cos \phi_{x b}\right)$.

Some remarks are in order:

(i) The final form confirms the UV-safe solution of the differential equation (2.15) of [13].

(ii) The simple expression of the solution in the rhs of Eq. (C9) has the same form of the phase factors in the integral representation (C1) coming from $\mathrm{H}$-diagram vertices, evaluated at the angle $\phi_{A B}=$ $\phi_{x, x-b}$. In particular, the $h_{T T}$ component has a geometrical significance, embodied in the relation

$$
\frac{\sin \phi_{A B}}{b}=\frac{\boldsymbol{x} \wedge(\boldsymbol{x}-\boldsymbol{b})}{b|\boldsymbol{x} \| \boldsymbol{x}-\boldsymbol{b}|}=\frac{2 \text { Area }}{|\boldsymbol{x} \| \boldsymbol{x}-\boldsymbol{b}| b} .
$$

\section{Calculation of the $\boldsymbol{h}$ field in momentum space}

The two-dimensional Fourier transform of the complex field $h(\boldsymbol{b}, \boldsymbol{x})$ with respect to the transverse variable $\boldsymbol{x}$ is given by

$$
\begin{aligned}
\tilde{h}(\boldsymbol{b}, \boldsymbol{q}) & \equiv \int \mathrm{d}^{2} \boldsymbol{x} \mathrm{e}^{\mathrm{i} \boldsymbol{q} \cdot \boldsymbol{x}} h(\boldsymbol{b}, \boldsymbol{x}) \\
& =\frac{1}{2 \pi^{2} \boldsymbol{q}^{2}} \int \mathrm{d}^{2} \boldsymbol{q}_{2} \mathrm{e}^{\mathrm{i} \boldsymbol{q}_{2} \cdot \boldsymbol{b}}\left[1-\mathrm{e}^{2 \mathrm{i} \phi_{12}}\right] .
\end{aligned}
$$

In fact, by replacing $h(\boldsymbol{b}, \boldsymbol{x})$ with the integral representation (C1), the integration in $\boldsymbol{x}$ just provides a delta function $\delta^{2}\left(\boldsymbol{q}-\boldsymbol{q}_{1}-\boldsymbol{q}_{2}\right)$ which is then used to perform the integration in $\boldsymbol{q}_{1}=\boldsymbol{q}-\boldsymbol{q}_{2}$, according to Eqs. (2.11)-(2.12) of [13].

By introducing the complex variables

$$
q \equiv q_{x}+\mathrm{i} q_{y}, \quad q_{2} \equiv q_{2 x}+\mathrm{i} q_{2 y}
$$

the angular factors can be written in rational form: 


$$
\begin{aligned}
\mathrm{e}^{2 \mathrm{i} \phi_{1}} & =\frac{q_{1}}{q_{1}^{*}}=\frac{q-q_{2}}{\left(q-q_{2}\right)^{*}}, \quad \mathrm{e}^{-2 \mathrm{i} \phi_{2}}=\frac{q_{2}^{*}}{q_{2}} \\
1-\mathrm{e}^{2 \mathrm{i} \phi_{12}} & =1-\frac{q-q_{2}}{\left(q-q_{2}\right)^{*}} \frac{q_{2}^{*}}{q_{2}}=\frac{q_{2} q^{*}-q_{2}^{*} q}{q_{2}\left(q-q_{2}\right)^{*}} .
\end{aligned}
$$

Without loss of generality we can orient the impactparameter vector along the real axis: $\boldsymbol{b}=(b, 0)$. In this way $\boldsymbol{q}_{2} \cdot \boldsymbol{b}=q_{2 x} b$ and we obtain

$$
\begin{aligned}
2 \pi^{2} \tilde{h} & =\frac{1}{q} \int \mathrm{d}^{2} \boldsymbol{q}_{2} \frac{\mathrm{e}^{\mathrm{i} q_{2 x} b}}{q^{*}-q_{2}^{*}}-\frac{1}{q^{*}} \int \mathrm{d}^{2} \boldsymbol{q}_{2} \frac{\mathrm{e}^{\mathrm{i} q_{2 x} b} q_{2}^{*}}{q_{2}\left(q^{*}-q_{2}^{*}\right)} \\
& \equiv \frac{I_{1}}{q}-\frac{I_{2}}{q^{*}} .
\end{aligned}
$$

The first integral is straightforward:

$$
\begin{aligned}
I_{1} & =\int_{\mathbb{R}^{2}} \mathrm{~d} q_{2 y} \mathrm{~d} q_{2 x} \frac{\mathrm{e}^{\mathrm{i} q_{2 x} b}}{q^{*}-q_{2 x}+\mathrm{i} q_{2 y}} \\
& =-2 \pi \mathrm{i} \mathrm{e}^{\mathrm{i} q^{*} b} \int_{-\infty}^{+\infty} \mathrm{d} q_{2 y} \Theta\left(q_{2 y}-q_{y}\right) \mathrm{e}^{-q_{2 y} b} \\
& =-2 \pi \mathrm{i} \frac{\mathrm{e}^{\mathrm{i} q_{x} b}}{b}
\end{aligned}
$$

where the $q_{2 x}$ integral has been performed by closing the contour in the upper complex half plane, where the simple pole at $q_{2 x}=q^{*}+\mathrm{i} q_{2 y}$ is found provided $q_{2 y}-q_{y}>0$.

Also in the second integral of Eq. (C15) the $q_{2 x}$ integration is performed in the upper complex half plane, where two simple poles can be found: the previous one and another one at $q_{2 x}=-\mathrm{i} q_{2 y}$ provided $q_{2 y}<0$. Explicitly

$$
\begin{aligned}
I_{2}= & \int_{\mathbb{R}^{2}} \mathrm{~d} q_{2 y} \mathrm{~d} q_{2 x} \frac{\mathrm{e}^{\mathrm{i} q_{2 x} b}\left(q_{2 x}-\mathrm{i} q_{2 y}\right)}{\left(q_{2 x}+\mathrm{i} q_{2 y}\right)\left(q^{*}-q_{2 x}+\mathrm{i} q_{2 y}\right)} \\
= & 2 \pi \mathrm{i} \int_{-\infty}^{+\infty} \mathrm{d} q_{2 y}\left\{\Theta\left(-q_{2 y}\right) \frac{-2 \mathrm{i} q_{2 y} \mathrm{e}^{q_{2 y} b}}{q^{*}+2 \mathrm{i} q_{2 y}}\right. \\
& \left.-\Theta\left(q_{2 y}-q_{y}\right) \frac{q^{*} \mathrm{e}^{\mathrm{i} q^{*} b} \mathrm{e}^{-q_{2 y} b}}{q^{*}+2 \mathrm{i} q_{2 y}}\right\} \\
= & 2 \pi \mathrm{i}\left\{-\frac{1}{b}+q^{*}\left[\int_{0}^{\infty} \frac{\mathrm{e}^{-t} \mathrm{~d} t}{q^{*} b-2 \mathrm{i} t}-\mathrm{e}^{\mathrm{i} q_{x} b} \int_{0}^{\infty} \frac{\mathrm{e}^{-t} \mathrm{~d} t}{q b+2 \mathrm{i} t}\right]\right\},
\end{aligned}
$$

where we made the substitutions $t=-q_{2 y} b$ and $t=$ $\left(q_{2 y}-q_{y}\right) b$ in the two integrals of Eq. (C17), respectively. The latter are related to the exponential-integral special function $E_{1}$ defined by

$$
E_{1}(z) \equiv \int_{z}^{+\infty} \frac{\mathrm{e}^{-t}}{t} \mathrm{~d} t, \quad(|\arg (z)|<\pi)
$$

so that, by combining Eqs. (C15)-(C17) we finally obtain $\tilde{h}(\boldsymbol{q} ; \boldsymbol{b})=\frac{\mathrm{i}}{\pi b}\left(\frac{1}{q^{*}}-\frac{\mathrm{e}^{\mathrm{i} q_{x} b}}{q}\right)+\frac{\mathrm{e}^{\mathrm{i}} q^{*} b}{2 \pi}\left[E_{1}\left(\frac{\mathrm{i} q^{*} b}{2}\right)+E_{1}\left(-\frac{\mathrm{i} q b}{2}\right)\right]$.

We notice that $\tilde{h}$ obeys a simple conjugation property, $\tilde{h}^{*}=\mathrm{e}^{-\mathrm{i} q \cdot \boldsymbol{b}} \tilde{h}$, and therefore the combination

$$
\mathrm{e}^{-\frac{\mathrm{i}}{2} \boldsymbol{q} \cdot \boldsymbol{b}} \tilde{h}=\left[\mathrm{e}^{-\frac{\mathrm{i}}{2} q \cdot \boldsymbol{b}} \tilde{h}\right]^{*} \in \mathbb{R}
$$

is real valued, and reads

$$
\begin{aligned}
\mathrm{e}^{-\frac{\mathrm{i}}{2} q \cdot b \tilde{h}} & =\frac{\mathrm{ie}^{-\frac{\mathrm{i}}{2} q_{x} b}}{\pi q^{*} b}+\frac{\mathrm{e}^{-\frac{1}{2} q_{y} b}}{2 \pi} E_{1}\left(\frac{\mathrm{i} q^{*} b}{2}\right)+\text { c.c. } \\
& =\frac{\mathrm{i}}{\pi} \mathrm{e}^{-\frac{\mathrm{i}}{2} q_{x} b}\left[\frac{1}{q^{*} b}-\int_{0}^{\infty} \frac{\mathrm{e}^{-t} \mathrm{~d} t}{q^{*} b-2 \mathrm{i} t}\right]+\text { c.c. } \\
& =\frac{2}{\pi} \mathrm{e}^{-\frac{\mathrm{i}}{2} q_{x} b} \int_{0}^{\infty} \frac{\mathrm{e}^{-t} \mathrm{~d} t}{\left(q^{*} b-2 \mathrm{i} t\right)^{2}}+\text { c.c. } \\
& =\frac{2}{\pi q^{*} b} \mathrm{e}^{-\frac{\mathrm{i}}{2} q_{x} b} \int_{0}^{\infty} \frac{t \mathrm{e}^{-t} \mathrm{~d} t}{q^{*} b-2 \mathrm{i} t}+\text { c.c. }
\end{aligned}
$$

where integrations by parts have been performed in the last steps.

\section{APPENDIX D: AN ARGUMENT FOR THE CUTOFF $\omega_{\max } \sim R^{-1} \Theta_{s}^{-2}$}

In this appendix we repeat, in more explicit terms, the argument advocated in [31] for an upper cutoff on the $\omega$ spectrum. To this purpose, we should write the frequency spectrum of the emitted energy in terms of the so-called news functions as

$$
\frac{d E}{d \omega} \propto|c(\omega)|^{2}
$$

and the energy emitted per unit retarded time $u \sim t-r$ as

$$
\frac{d E}{d u} \propto|\tilde{c}(u)|^{2}
$$

where $c$ and $\tilde{c}$ are one-dimensional Fourier transforms of each other. From Eqs. (D1)-(D2) we have, up to numerical constants,

$c(\omega) \sim \sqrt{G s} \Theta_{s} \log ^{\frac{1}{2}}\left(\frac{1}{\omega R}\right), \quad\left(\omega<R^{-1}\right) ;$

$c(\omega) \sim \sqrt{G s} \Theta_{s}(\omega R)^{-\frac{1}{2},}, \quad\left(\omega>R^{-1}\right)$,

and we find

$\tilde{c}(u) \sim \sqrt{G s} \Theta_{s} u^{-1} \log ^{\mp \frac{1}{2}}\left(\frac{u}{R}\right), \quad(u>R) ;$

$\tilde{c}(u) \sim \sqrt{G s} \Theta_{s}(u R)^{-\frac{1}{2},} \quad(u<R)$,

where the $-(+)$ holds for the even (odd) part of $\tilde{c}(\omega)$ under $\omega \rightarrow-\omega$. 
We thus get the following power time history for GW emission:

$$
\begin{aligned}
& \frac{\mathrm{d} E^{\mathrm{GW}}}{\mathrm{d} u}=G s \Theta_{s}^{2} u^{-2} \log ^{\mp 1}(u / R), \quad(u>R) ; \\
& \frac{\mathrm{d} E^{\mathrm{GW}}}{\mathrm{d} u}=G s \Theta_{s}^{2}(u R)^{-1}, \quad(u<R) .
\end{aligned}
$$

At this point we note that the latter behavior exceeds a generally believed (so-called Dyson) bound on the maximal power in gravitational-wave energy emission (see e.g. [40]): $P_{\mathrm{GW}} \leq c^{5} G_{N}^{-1} \rightarrow 1$ in our units, if $u<R \Theta_{s}^{2}$. But this precisely corresponds to saying that for $\omega>R^{-1} \Theta_{s}^{-2}$ the spectrum should soften in order for the bound on the power to be respected at very early times after the collision.
[1] G. 't Hooft, Phys. Lett. B 198, 61 (1987).

[2] I. J. Muzinich and M. Soldate, Phys. Rev. D 37, 359 (1988).

[3] D. Amati, M. Ciafaloni, and G. Veneziano, Phys. Lett. B 197, 81 (1987).

[4] D. Amati, M. Ciafaloni, and G. Veneziano, Int. J. Mod. Phys. A 03, 1615 (1988).

[5] H. L. Verlinde and E. P. Verlinde, Nucl. Phys. B371, 246 (1992).

[6] D. Amati, M. Ciafaloni, and G. Veneziano, Nucl. Phys. B347, 550 (1990).

[7] D. Amati, M. Ciafaloni, and G. Veneziano, Nucl. Phys. B403, 707 (1993).

[8] P. Aichelburg and R. Sexl, Gen. Relativ. Gravit. 2, 303 (1971).

[9] M. Ciafaloni and D. Colferai, J. High Energy Phys. 10 (2014) 85.

[10] X. O. Camanho, J. D. Edelstein, J. Maldacena, and A. Zhiboedov, arXiv:1407.5597.

[11] G. D’Appollonio, P. Vecchia, R. Russo, and G. Veneziano, J. High Energy Phys. 05 (2015) 144.

[12] L. Lipatov, Zh. Eksp. Teor. Fiz. 82, 991 (1982).[Sov. Phys. JETP 55, 582 (1982)].

[13] D. Amati, M. Ciafaloni, and G. Veneziano, J. High Energy Phys. 02 (2008) 049.

[14] G. Marchesini and E. Onofri, J. High Energy Phys. 06 (2008) 104.

[15] G. Veneziano and J. Wosiek, J. High Energy Phys. 09 (2008) 023.

[16] G. Veneziano and J. Wosiek, J. High Energy Phys. 09 (2008) 024.

[17] D. M. Eardley and S. B. Giddings, Phys. Rev. D 66, 044011 (2002).

[18] E. Kohlprath and G. Veneziano, J. High Energy Phys. 06 (2002) 057.
[19] H. Yoshino and Y. Nambu, Phys. Rev. D 67, 024009 (2003).

[20] S. B. Giddings and V. S. Rychkov, Phys. Rev. D 70, 104026 (2004).

[21] M. Ciafaloni and D. Colferai, J. High Energy Phys. 11 (2008) 047.

[22] M. Ciafaloni and D. Colferai, J. High Energy Phys. 12 (2009) 062.

[23] M. Ciafaloni, D. Colferai, and G. Falcioni, J. High Energy Phys. 09 (2011) 044.

[24] G. F. Giudice, R. Rattazzi, and J. D. Wells, Nucl. Phys. B630, 293 (2002).

[25] V. S. Rychkov (private communication).

[26] L. Smarr, Phys. Rev. D 15, 2069 (1977).

[27] P. D. D'Eath, Phys. Rev. D 18, 990 (1978).

[28] P. D. D'Eath and P. N. Payne, Phys. Rev. D 46, 658 (1992).

[29] S. J. Kovacs and K. S. Thorne, Astrophys. J. 217, 252 (1977).

[30] S. J. Kovacs and K. S. Thorne, Astrophys. J. 224, 62 (1978).

[31] A. Gruzinov and G. Veneziano, arXiv:1409.4555.

[32] P. Spirin and T. N. Tomaras, J. High Energy Phys. 06 (2015) 153.

[33] S. W. Hawking, Commun. Math. Phys. 43, 199 (1975).

[34] S. W. Hawking, arXiv:1509.01147.

[35] M. Ciafaloni, D. Colferai, and G. Veneziano, Phys. Rev. Lett. 115, 171301 (2015).

[36] G. Dvali, C. Gomez, R. Isermann, D. Lüst, and S. Stieberger, Nucl. Phys. B893, 187 (2015).

[37] D. J. Gross and P. F. Mende, Phys. Lett. B 197, 129 (1987).

[38] S. Weinberg, Phys. Rev. 140, B516 (1965).

[39] M. Jacob and G. C. Wick, Ann. Phys. (N.Y.) 7, 404 (1959); M. Jacob and G. C. Wick, Ann. Phys. (N.Y.) 281, 774 (2000).

[40] V. Cardoso, Gen. Relativ. Gravit. 45, 2079 (2013). 